
\title{
Library
}

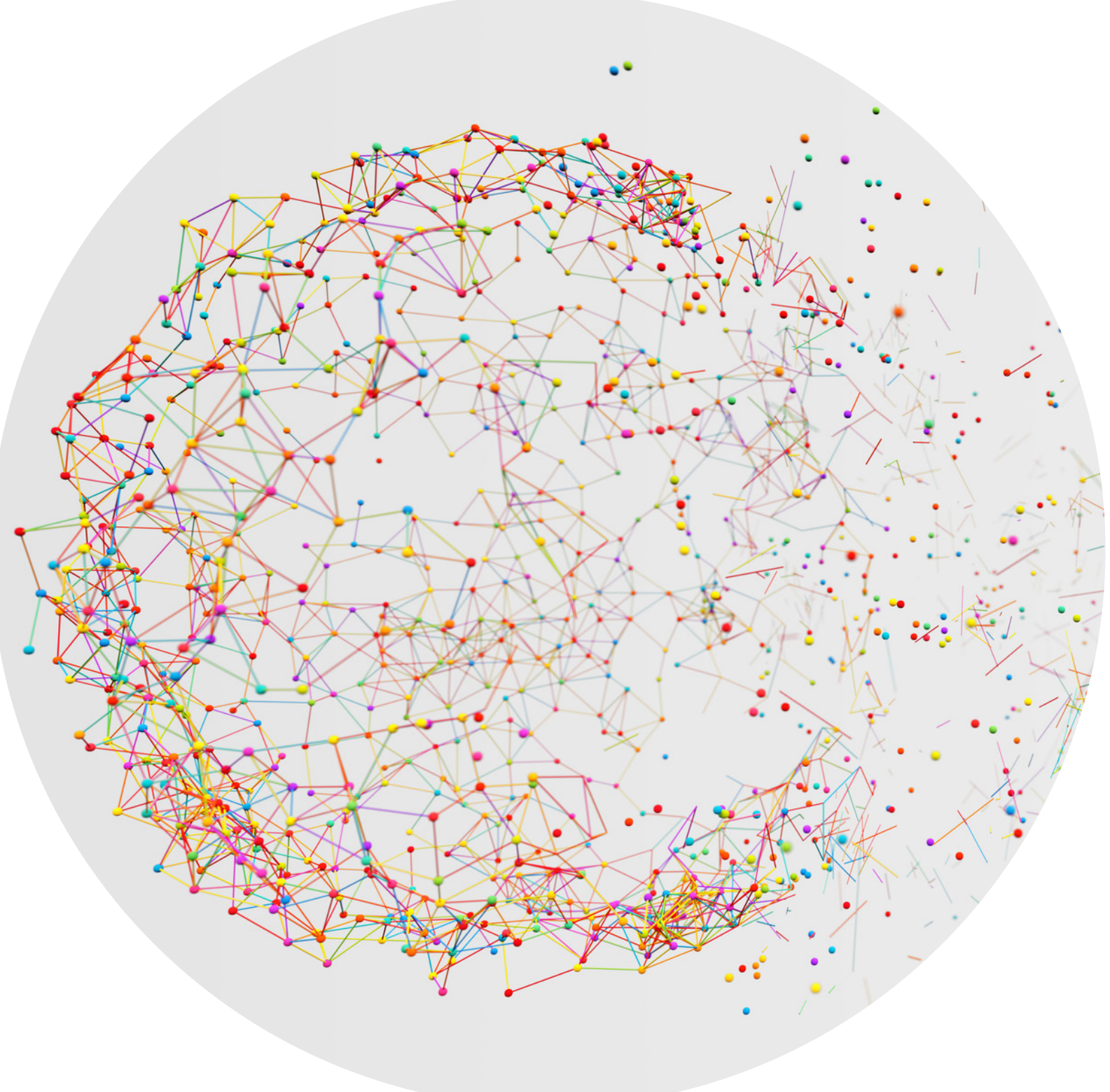

\section{Collaborating in response to COVID-19: editorial and methods initiatives across Cochrane}




\section{Contents}

Introduction

\section{Editorial}

COVID-19: working together and making a difference for decision-makers

\section{Coordinating and preparing for evidence synthesis}

COVID-END: an international network to better co-ordinate and maximise the impact of the global evidence synthesis and guidance response to COVID-19

CEOsys: Creating an ecosystem for COVID-19 evidence

Prioritization during a pandemic: taking a strategic approach for a sustained response 12

Developing a core outcome set for COVID-19 prevention interventions: the COS COVID-P study 15

Cochrane's COVID-19 consumer rapid response group

\section{Facilitating and developing methods for evidence synthesis}

COVID-NMA: a collaborative COVID-19 living evidence project

Cochrane COVID-19 living systematic reviews: why, how, and what has been done?

Rapid review methods guidance aids in Cochrane's quick response to the COVID-19 crisis

Developing rapid reviews of diagnostic tests in the time of COVID-19: current knowledge and future steps

Rapid development of the Cochrane COVID-19 Study Register to support review production

\section{Organizing collaborative approaches for evidence synthesis and publication}

Learning from emergency trauma teams: an organizational approach for conducting (very) rapid reviews

Fostering international co-operation and junior researchers' engagement during a pandemic: InterNetCOVID-19

The Central Editorial Service: a collaborative editorial process for publishing high-priority Cochrane Reviews

The Public Health Hub: an adaptive model for rapid publication of high-priority reviews

\section{Ensuring evidence informs practice and guidelines}

The eCOVID-19 living recommendations map and gateway to contextualisation

International collaborations with Cochrane Oral Health to inform the re-opening of dental practices

Cochrane ENT in the COVID-19 pandemic: using our expertise and collaborating effectively

Cochrane Childhood Cancer and COVID-19: developing recommendations for childhood, adolescent and young adult cancer survivors

Collaborating to mobilize, update, and promote Cochrane evidence to support intensive care in the

COVID-19 pandemic

REH-COVER (Rehabilitation-COVID-19 Evidence-based Response) action to recover functioning during/after COVID-19 and its treatments

COVID-19 Emergency Evidence Response Service: report from Ireland 


\section{Introduction}

Cochrane provides high-quality, relevant and up-to-date synthesized research evidence to inform health decisions. With the emergence of the COVID-19 pandemic, health decision-makers needed trustworthy evidence to help answer many questions, and they needed it quickly. During 2020, the Cochrane community worked with partners to find ways to respond to this situation and meet the needs of evidence users.

This Supplement to the Cochrane Database of Systematic Reviews collects inititatives involving Cochrane groups, in the form of short reports. Each short report details the activities and experiences from Cochrane groups and their partners, highlighting the challenges and questions their projects addressed, the processes and methods used, and their findings or outcomes. The reports also consider the lessons learned throughout the project and highlight recommendations that will help Cochrane and the wider community with future preparedness.

With thanks to the many individuals and groups who contributed to work on producing and using evidence in the global response to the COVID-19 pandemic.

www.cochranelibrary.com

Supplement produced by Cochrane and John Wiley \& Sons Ltd.

\section{Cochrane:}

Editor: Ella Flemyng

Senior Editor/Project Lead: John Hilton

Assistant Editor: Justin Mann

Copy Editor: Denise Mitchell

\section{Wiley:}

Editorial consultants: Tony Aburrow, Cathryn Fowler

Production manager: David Hives

Copyright $\odot 2020$ The Cochrane Collaboration. Published by John Wiley \& Sons, Ltd.

Cite this supplement as:

Cochrane. Collaborating in response to COVID-19: editorial and methods initiatives across Cochrane. Cochrane Database of Systematic Reviews 2020;(12 Suppl 1). https://doi.org/10.1002/14651858.CD202002

Cite individual articles as:

[Authors]. [Title]. In: Collaborating in response to COVID-19: editorial and methods initiatives across Cochrane. Cochrane Database of Systematic Reviews 2020;(12 Suppl 1):[page numbers]. https://doi.org/10.1002/14651858.CD202002 


\title{
COVID-19: working together and making a difference for decision-makers
}

\author{
Authors: John Hilton ${ }^{1}$, Ella Flemyng ${ }^{1}$, Karla Soares-Weiser ${ }^{1}$ \\ This is an abridged version of an Editorial published in the Cochrane Database of Systematic Reviews. Read the full \\ version at cochranelibrary.com/cdsr/doi/10.1002/14651858.ED000150
}

With the emergence of COVID-19 in early 2020, Cochrane started planning its response. The first steps were to set up a working group of members of the Cochrane community and to start discussions with key partners. Cochrane's mission is to promote evidenceinformed health decision-making by producing high-quality, relevant, accessible systematic reviews and other synthesized research evidence. As such, Cochrane's response to COVID-19 had to meet the needs of evidence users and decision-makers as the pandemic developed.[1]

For Cochrane's central editorial team, the goal was to deliver high-quality systematic reviews, in a complex environment, at high speed. Accelerating the development and publication of key Cochrane Reviews posed multiple challenges. That learning process was valuable for setting strategic directions. Beyond the central team, the Cochrane community's diversity of thought, geographic locations, and multidisciplinary expertise were of paramount importance, and showed what can be achieved by engaging and working together towards a common goal.

The innovation, responsiveness, and diverse experiences of all these teams are demonstrated in a series of short reports collected in this special supplement to the Cochrane Database of Systematic Reviews. The supplement collects examples of initiatives across four broad areas:

- Coordinating and preparing for evidence synthesis

- Facilitating and developing methods for evidence synthesis

- Organizing collaborative approaches for evidence synthesis and publication

- Ensuring evidence informs guidelines and practice

The first set of reports in the supplement provide examples of how the evidence ecosystem can be better navigated to facilitate evidence synthesis. Reports highlight how to prioritize research questions during a pandemic (page 12) and how to collaborate and organize to maximize impact at national (page 9) and international levels (page 4). A consistent theme has been the need to reduce the heterogeneity of trials, such as through the development and use of core outcome sets (page 15). Cochrane's consumer rapid review response group also highlights how patient and public involvement can be implemented and embedded from the start in such a response (page 19).

Accelerated review processes for both intervention (page 28) and diagnostic test accuracy (page 32) questions have come to the forefront, helping organizations to make evidence-informed decisions quickly. When the evidence base changes frequently, living systematic reviews (page 25) and network meta-analysis (page 21) become key methods, alongside technological and organizational solutions. Many of the reports discuss approaches for dealing with scarce data, how to manage an evolving evidence base, and how to coordinate and streamline evidence surveillance. Technical solutions to collating primary research are central to this, with Cochrane's COVID-19 Study Register now widely used to support review development (page 37). And there is a clear call for a change in culture towards openness and sharing, including of research data and protocols.

Beyond methods and technical developments, organizational approaches to facilitate evidence synthesis and publication can increase the timeliness of review development without compromising on quality. Cochrane Austria's adaption of an emergency trauma team organizational structure to conduct a systematic review quickly is an example of how programme management and a strong team can combine to achieve results (page 41). Broader collaborative editorial processes, either through a centralized editorial service (page 47) or a 'hub' service (page 51), can be adapted for rapid publication of high-priority reviews, and the InterNetCOVID-19 project showed how senior and early career researchers can work together to support review development in times of a pandemic (page 44). Such services also benefit from the enthusiasm and willingness of volunteers, highlighting the potential for tools such as Cochrane's TaskExchange (taskexchange.cochrane. org), for engaging and coordinating collaborators.

${ }^{1}$ Cochrane, UK 


\section{Overarching themes}

Building flexibly on existing collaborations and nurturing those relationships in the long term

New structured approaches that can be incorporated into future working

Technological innovation facilitating new platforms and global team working

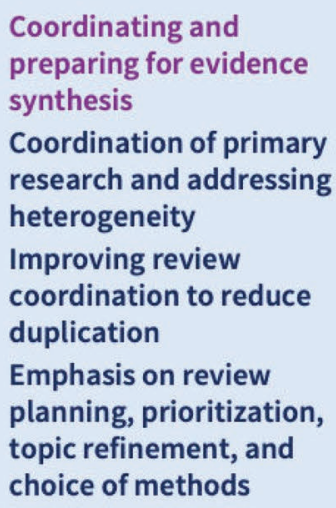

Figure 1. Emerging themes from the short reports

The final group of reports in the Supplement looks at how Cochrane groups have worked with national and international bodies to prioritize reviews, to inform the most urgent health questions and support guideline development. It includes reports on research in diverse clinical areas including ENT (page 61), oral health (page 58), childhood cancer (page 63), intensive care (page 65), rehabilitation (page 67), and pregnancy (pages 77), as well as regional examples from Ireland (page 72) and South Africa (page 79). Many of these projects built on existing expertise and structures, and were supported from funding from national and international collaborators seeking to answer high-priority questions. Taking this a step further, the eCOVID-19 living recommendations platform will provide hosts a comprehensive map of recommendations from high-quality guidelines for all stakeholders (page 54).

The main themes emerging are summarized in Figure 1. The need to be agile and to adapt is key. Cochrane looked to collaboration to respond to a crisis, but this was only possible because of Cochrane's network. The expertise found within the Cochrane community was also crucial. Keeping high-priority reviews updated requires resources and those should be directed at reviews of most importance to decision-makers. We must apply the lessons learned to our current editorial process and strive for transparency and independence in our processes. A key lesson has been the importance of engagement and constant communication, not just at times of crisis but also nurturing connections at other times.

Included in the reports in this supplement are examples of innovation that can inform future preparedness, and an appreciation of how that innovation is facilitated by pre-existing relationships within Cochrane and with partners. Momentum
Organizational approaches for evidence synthesis and publication

Balancing quality assurance with timeliness

Building on the enthusiasm and willingness of volunteers Ensuring diversity and inclusivity of teams
Ensuring evidence informs guidelines and practice

Working directly with international and regional bodies on prioritization Building on pre-existing expertise within communities Ensuring flexibility and understanding within teams Importance of funding

has been growing to transform the evidence ecosystem and how stakeholders work together within it, and the COVID-19 response has been a catalyst to move some of these plans into action.[2] Cochrane groups and their partners have provided examples of how to bring primary research, evidence synthesis, and guideline development together, via technological solutions and global teams. And there are many other examples of ongoing work not included in the supplement.[1] The health, societal, and economic impact of COVID-19 has been devastating, and Cochrane needs to learn from the experience of 2020 to be better prepared for future events. Cochrane's position and principles means it can help in leading, facilitating, and advocating for this work.

\section{Declarations of interest}

$\mathrm{JH}$ is a Senior Editor, KSW is the Editor in Chief, and EF is Methods Implementation Manager at Cochrane; all are full-time employees of Cochrane. The authors declare no additional interests.

\section{Acknowledgments}

With thanks to the many groups and individuals whose work is featured in this CDSR Supplement.

\section{References}

1. Cochrane. Coronavirus (COVID-19) - Cochrane resources and news. cochrane.org/coronavirus-covid-19-cochrane-resources-and-news (accessed 30 November 2020)

2. Ravaud P, Créquit P, Williams HC, Meerpohl J, Craig JC, Boutron I. Future of evidence ecosystem series: 3 . From an evidence synthesis ecosystem to an evidence ecosystem. Journal of Clinical Epidemiology 2020;123;153-61. https://doi.org/10.1016/j.jclinepi.2020.01.027 


\title{
COVID-END: an international network to better co-ordinate and maximize the impact of the global evidence synthesis and guidance response to COVID-19
}

\begin{abstract}
Authors: Jeremy M Grimshaw ${ }^{1}$, David I Tovey² ${ }^{2}$ John N Lavis ${ }^{3}$, on behalf of COVID-END
Participating groups: Cochrane, Cochrane Australia, Cochrane Ireland, International Initiative for Impact Evaluation (3ie), ACRES: The Centre for Rapid Evidence Synthesis, Agency for Clinical Innovation, New South Wales, AGREE Enterprise, Africa Centre for Evidence, The Africa Centre for Systematic Reviews \& Knowledge Translation, Africa Evidence Network, AHRQ evidence-based Care Transformation Support (ACTS) Initiative, Campbell Collaboration, Centre for Evidence-Based Health Care, Stellenbosch University, COVID-19 Knowledge Accelerator, COVID-NMA, eBASE Africa, Epistemonikos Foundation, EPPI-Centre, Evidence Aid, EUnetHTA, Evidence Based Medicine Centre, Lanzhou University, Nanjing University of Science and Technology, Evidence Synthesis International, Evidence Synthesis Ireland, Global Evidence Synthesis Initiative, Guidelines International Network, Health Information Research Unit, Health Technology Assessment International, Institute for Quality and Efficiency in Health Care, Institut National d'Excellence en Santé et en Services Sociaux, Joanna Briggs Institute, Knowledge to Policy Center American University of Beirut, MAGIC Evidence Ecosystem Foundation, McMaster Health Forum, Memorial University of Newfoundland and Labrador, Drug Safety and Effectiveness Network, National Collaborating Centre for Methods and Tools, National Institute for Health and Care Excellence, Norwegian Institute of Public Health, Oswaldo Cruz Foundation, Ottawa Hospital Research Institute, Oxford Centre for Evidence-based Medicine, PROSPERO, Pushpagiri Centre for Evidence Based Practice, South African Medical Research Council, SPOR Evidence Alliance, Trip Database, UNCOVER, Universiti Putra Malaysia
\end{abstract}

Corresponding Author: Jeremy Grimshaw: jgrimshaw@ohri.ca

\section{Introduction and background}

The COVID-19 pandemic is one of the greatest health and societal challenges that the world has collectively faced in many decades. Policy, public health, clinical and individual decision makers are actively seeking evidence and evidence-based guidance (e.g. health technology assessments, clinical practice guidelines) on prevention, management and mitigation of the health, social and economic impacts of COVID-19. As a result, there has been a dramatic global increase in basic and applied health (and to a lesser extent social and economic) research. Given that individual studies are rarely sufficient to guide policy, clinical and individual decisions, and the evidence base is rapidly evolving, decision makers need highquality, context-relevant evidence syntheses and trustworthy guidance more than ever. It was heartening then, to see a dramatic increase in evidence synthesis, guidance and associated decision support activities globally to meet the needs of the pandemic during the first half of 2020.

Many of the eventual partners in COVID-END were involved in some type of evidence synthesis, guidance and support activities when COVID-19 struck. These partners rapidly pivoted to focus their work on COVID-19. We (JMG, JNL) observed that during the initial stages of the pandemic, the evidence synthesis response largely focused on rapid reviews, which were rapidly out of date despite the ongoing relevance of the review question and were of variable quality, and that guidance development largely focused on expert opinion. There was also huge duplication of effort globally, creating a major noise to signal problem. We decided to convene a series of exploratory meetings in early April of the key global evidence synthesis, guidance and decision support organizations to see whether other groups shared our concerns.

${ }^{1}$ Ottawa Hospital Research Institute and University of Ottawa, Canada; ${ }^{2}$ COVID-END, UK; ${ }^{3}$ McMaster Health Forum, McMaster University, Canada 
We also explored whether there was an appetite to work together to promote collaboration, improve the efficiency of evidence synthesis and guidance production and decision support activities, and reduce inappropriate duplication of effort in order to maximize the impact of the global evidence response to COVID-19. Following these meetings, we established COVID-END: the COVID-19 Evidence Network to support Decision-making (covid-end.org/), as a time-limited network of global evidence synthesis, guidance and decision support organizations that aims better to co-ordinate their collective evidence response to the pandemic.

\section{Key activities and strategies}

We identified COVID-END partners - globally leading evidence synthesis, guidance and support organizations working on COVID-related activities (ensuring global representation) - that initially met virtually twice weekly. We established seven working groups (focusing on scoping, engaging, digitizing, synthesizing, recommending, packaging and sustaining activities) involving representatives from COVID-END partners. We rapidly established the scope of COVID-END and working principles, including the importance of global representation and equity in all aspects of our work. We recognized the need for evidence syntheses and guidance in four areas (addressing public health, clinical management, health system arrangements, and economic and social issues).

We asked the working groups to identify short-term projects that 'in weeks' could be helpful. For example, the synthesizing group created an interactive flow-chart featuring resources for groups thinking of doing evidence syntheses, encouraging them to clarify the issue or decision to be informed, check to see that other groups had not already done the evidence synthesis (or if there was an ongoing synthesis), to register any new proposed reviews, and to use state-of-the-science approaches if updating an out-of-date review or conducting a new review. The packaging group provided principles for evidence packaging, targeting different stakeholder groups. The recommending group worked to identify and share standards, methods, processes and digital platforms for developing, disseminating, adapting and implementing trustworthy, actionable and living guidance (linked to evidence). Low- and middle-income country (LMIC)-based partners highlighted the distinct challenges faced by evidence synthesis and support organisations in LMIC settings.[1] The COVID-END secretariat created a guide of key COVID-19 evidence sources, which could be rapidly scanned to identify best current evidence and an evidence-support model for producing rapid evidence profiles within four hours.

All these resources (and more) are freely available on the COVIDEND website (covid-end.org; see 'Additional Resources' section for the URLS of all COVID-END resources).

By June 2020, it became obvious that COVID-19 was not going to be a sprint that would be over in months but something that is likely to be with us for the foreseeable future. Given this, we became convinced that the initial evidence synthesis, guidance and decision support response was not sustainable and that there were opportunities to rethink the model to increase the signal-tonoise ratio and improve value for decision makers, and quality and efficiency of synthesis and guidance production.

Specifically, we argue that the world will be best served by:

- better co-ordination and collaboration within the evidence ecosystem;[2]

- a global stock of high-quality, open-access living systematic reviews covering $(80 \%$ of) priority issues (public health measures, clinical management, health system arrangements, economic and social responses) faced by decision makers (to allow them to focus on contextualization of evidence within their setting) available in multiple languages;

- trustworthy, accessible and living guidance covering key (public health measures, clinical management, health system arrangements, economic and social responses) issues faced by decision makers;

- evidence synthesis capacity to undertake priority syntheses where high-quality, living systematic reviews are not available;

- increased exploitation of technology to increase the speed of production and to support knowledge translation;

- international, national and local decision-support initiatives that can support policy and other decision makers to find and interpret best evidence and guidance;

- global evidence eco-system infrastructure (building wherever possible on existing evidence synthesis, guidance and support initiatives, e.g., PROSPERO to register ongoing evidence syntheses) to encourage interoperability and facilitate efficient conduct and sharing of evidence syntheses, guidance and trustworthy decision support; and

- secure funding to support these activities.

Currently we are undertaking a number of projects that 'in months' could be helpful including:

- an inventory of best evidence syntheses linked to specific policy decisions, where high-quality, up-to-date (preferably living) syntheses will be identified making it even easier for decision makers to find the best evidence;

- a horizon-scanning panel of key global stakeholders that meets monthly to identify recurrent and emerging issues where syntheses would be needed in the coming months;

- a prioritisation process to identify where living systematic reviews are most needed, using gaps in the inventory and insights from the horizon-scanning panel, which can be used to encourage groups to take responsibility collectively for a full set of living reviews addressing all priority issues related to the pandemic and pandemic response; and 
- developing a virtual COVID-END community of any interested groups globally conducting evidence syntheses, guidance and decision support related to COVID-19.

We are keen to get feedback on any of these ideas and encourage readers to join the COVID-END Community if they are interested to learn more about our ongoing work and contribute to it.

Our aim was to work alongside major national and international organizations that were already established. We recognized Cochrane as the pre-eminent international health evidence synthesis organization that was conducting important COVID-19 reviews and producing valuable resources (such as the COVID-19 Register of Studies).[3] Given this, we were delighted when Cochrane was supportive of the COVID-END initiative. Senior members of Cochrane groups and secretariat have participated in all aspects of COVID-END's work. COVID-END's work has benefited from the methods, tools and approaches that Cochrane has established over the last two decades. The Cochrane Consumer group is helping COVID-END to identify individuals and groups that can support the involvement of citizens in the global evidence synthesis and guidance response for COVID-19.[4] In return, we believe COVID-END adds value by amplifying the work of Cochrane, broadening the network of evidence synthesis organizations in regular contact, and building on existing and facilitating new collaborations (including guidance organizations). Cochrane has also used COVID-END resources such as our taxonomy of decisions in its own priority-setting processes (covidreviews.cochrane.org/ prioritization).[5]

\section{Outcomes and impacts of activities}

COVID-END now has 49 partners covering all geographic regions globally. It has rapidly become recognised for its co-ordinating function of the global evidence synthesis, guidance and decision support response. We are aware of many groups globally who use COVID-END resources on a daily basis to support their activities (e.g. the World Health Organization (WHO) uses the COVID-END inventory of trustworthy sources to prepare rapid evidence briefs). Over 250 individuals have joined the virtual COVID-END Community. WHO requested COVID-END and Cochrane to join its secretariat function for the WHO Evidence Collaborative for COVID-19.

\section{Lessons for the future: sustainability and transferability}

COVID-END has brought together many of the key evidence synthesis, guidance and decision support organisations globally to work together to address the evidence needs of the pandemic. Many of the challenges we identified have been exacerbated by the pandemic but are not new.[6] The need for improved global co-ordination of evidence synthesis and guidance activities has been particularly evident for some time. Whilst COVID-END is a time-limited initiative, we hope that the lessons learned from this work will lead to a better global evidence eco-system, moving to optimally co-ordinated evidence production linked to living evidence and guidance. To increase the likelihood of this happening, the sustaining working group is evaluating the processes and outcomes of COVID-END (and their implications beyond the pandemic).

\section{Additional resources}

All of COVID-END's work is available through its website (COVID-END. org). In this report, we have highlighted a number of specific resources that readers might find of interest, including the following resources.

\section{Resources about COVID-END:}

- List of COVID-END partners: mcmasterforum.org/networks/covidend/overview/partners

- COVID-END's scope: mcmasterforum.org/networks/covid-end

- COVID-END principles: mcmasterforum.org/networks/covid-end/ overview/principles

- COVID-END Community (including details of how to join): mcmasterforum.org/networks/covid-end/resources-to-supportdecision-makers/covid-end-community

(Supply side) resources for groups undertaking evidence syntheses:

- Horizon-scanning panel: mcmasterforum.org/networks/covid-end/ resources-to-support-decision-makers/horizon-scanning-panel

- Resources for groups planning to undertake evidence syntheses: mcmasterforum.org/networks/covid-end/resources-for-researchers/ supports-for-evidence-synthesizers/interactive-flow-diagram

(Demand side) resources for groups supporting evidence use by decision makers:

- Guide to key COVID-19 evidence sources: mcmasterforum.org/ networks/covid-end/resources-to-support-decision-makers/guideto-key-covid-19-evidence-sources

- Inventory of best evidence syntheses: mcmasterforum.org/ networks/covid-end/resources-to-support-decision-makers/ Inventory-of-best-evidence-syntheses/context

- Evidence support models: mcmasterforum.org/networks/covid-end/ resources-to-support-decision-makers/evidence-support-models

- Evidence packaging resources: mcmasterforum.org/networks/ covid-end/resources-to-support-decision-makers/evidencepackaging-resources

\section{Acknowledgements}

We acknowledge the COVID-END partners and individuals from them that have contributed to COVID-END's work. 


\section{COVID-END}

Contributors include: Al-Khateeb S (RISE, McMaster Health Forum, McMaster University, Canada); Akl EA (Clinical Research Institute, American University of Beirut, Lebanon); Barreto JM (Oswaldo Cruz Foundation (FIOCRUZ), Brazil); Bai Z (Evidence-based Social Science and Health Research Center, Nanjing University of Science and Technology, China); Brandt L (MAGIC Evidence Ecosystem Foundation, Norway); Brassey J (Trip Database, UK); Boutron I (Centre of Research in Epidemiology and Statistics, Université de Paris, France); Bullock HL (RISE, McMaster Health Forum, McMaster University, Canada); Burke NN (Cochrane Ireland and Evidence Synthesis Ireland, Ireland); Chen Y (Evidence Based Medicine Centre, Lanzhou University, China); Ciurea I (McMaster Health Forum, McMaster University, Canada); Dieyi NU (Memorial University of Newfoundland and Labrador, Canada); de Haan S (Cochrane, Netherlands); Dion A (RISE, Ottawa Hospital Research Institute (OHRI), Canada); Dobbins M (National Collaborating Centre for Methods and Tools, McMaster University, Canada); El-Jardali $\mathrm{F}$ (Knowledge to Policy (K2P) Center | SPARK, American University of Beirut, Lebanon); Elliott JH (Cochrane Australia, Australia); Florez ID (University of Antioquia, Colombia); Gough D (EPPI-Centre, University College London, UK); Greenhalgh T (Interdisciplinary Research In Health Sciences Research Group, University of Oxford, UK); Henry L (Lucy Henry Consulting, Canada); lorio A (Department of Health Research Methods, Evidence and Impact, McMaster University, Canada); Kamga EB (Effective Basic Services (eBASE) Africa, Cameroon); Kawala BA (Africa Centre for Systematic Reviews and Knowledge Translation (MakCHS), Makerere University, Uganda); Kawooya I (ACRES - The Centre for Rapid Evidence Synthesis, Makerere University College of Health Sciences, Uganda); Kayongo E (ACRES - The Centre for Rapid Evidence Synthesis, Makerere University College of Health Sciences, Uganda); Kredo T (South African Medical Research Council, South Africa); Langer $L$ (Africa Centre for Evidence (ACE), University of Johannesburg, South Africa); Lewin S (Cochrane EPOC, Norwegian Institute of Public Health, Norway; South African Medical Research Council, South Africa); Lewis SZ (EBQ Consulting, LLC, USA); Lockwood C (JBI, Faculty of Health and Medical Sciences, University of Adelaide, Australia); Lotfi T (Global Evidence Synthesis Initiative, American University of Beirut, Lebanon; Health Research Methods, Evidence \& Impact, McMaster University, Canada); Mavergames C (Cochrane, Germany); McCaul M (Centre for Evidence Based Health Care, Department of Global Health, Stellenbosch University, South Africa); McQuillan R (Usher Institute, University of Edinburgh, UK); Mijumbi-Deve R (ACRES - The Centre for Rapid Evidence Synthesis, Makerere University College of Health Sciences, Uganda); Moat KA (McMaster Health Forum, McMaster University, Canada); Munn Z (Guidelines International Network (GIN), Scotland; $\mathrm{JBI}$, Faculty of Health and Medical Sciences, University of Adelaide, Australia); Nakibuuka LS (Memorial University of Newfoundland and Labrador, Canada); Ndagire R (Africa Centre for Systematic Reviews and Knowledge Translation (MakCHS), Makerere University, Uganda); Nunan D (Oxford Centre for Evidence-Based Medicine (CEBM), University of Oxford, UK); Obuku EA (Africa Centre for Systematic Reviews and Knowledge Translation (MakCHS), Makerere University, Uganda); Ocan M (Africa Centre for Systematic Reviews and Knowledge Translation (MakCHS), Makerere University, Uganda); Okwen PM (Effective Basic Services (eBASE) Africa, Cameroon); Oliver SR (EPPI-Centre, University College London, UK); Ooi CP (Universiti Putra Malaysia, Malaysia); Osheroff JA (TMIT Consulting and Chair, AHRQ evidence-based Care
Transformation Support (ACTS) Initiative COVID-19 Guidance to Action Collaborative, USA); Rada G (Epistemonikos Foundation, Chile); Reveiz L (Pan American Health Organization, USA); Roy D (Institut National d'Excellence en Santé et en Services Sociaux (INESSS), Canada); Smith M (Cochrane consumer, Canada); Snilstveit B (International Initiative for Impact Evaluation (3ie), UK); Stewart LA (Centre for Reviews and Dissemination, University of York, UK); Stewart R (Africa Centre for Evidence (ACE), University of Johannesburg, South Africa); Sutherland K (Agency for Clinical Innovation, New South Wales (ACI-NSW), Australia); Taske NL (National Institute for Health and Care Excellence (NICE), UK); Taylor BH (Evidence Aid, UK); Thomas J (EPPI-Centre, University College London, UK); Tricco AC (Li Ka Shing Knowledge Institute of St. Michael's Hospital, Unity Health Toronto, Canada); Vandvik PO (MAGIC Evidence Ecosystem Foundation, Norway); Vist GE (Norwegian Institute of Public Health, Norway); Wangi NR (Africa Centre for Systematic Reviews and Knowledge Translation (MakCHS), Makerere University, Uganda); Welch VA (Campbell Collaboration, Canada); Wild C (European Network for Health Technology Assessment (EUnetHTA), Austria); Young $\mathrm{T}$ (Centre for Evidence Based Health Care, Department of Global Health, Stellenbosch University, South Africa); Yu X (Evidence Based Medicine Centre, Lanzhou University, China); Zhu Y (Evidence Based Medicine Centre, Lanzhou University, China).

\section{Declarations of interest}

Jeremy Grimshaw published his first systematic review in 1993. He has been active in the Cochrane Collaboration in multiple roles since 1994 and has worked with many evidence synthesis organizations worldwide. $\mathrm{He}$ is currently the President of the Board of the Campbell Collaboration and Co-Chair of Evidence Synthesis International. David Tovey was the Editor in Chief of the Cochrane Library. John Lavis is a member of the Cochrane Editorial Board and the Cochrane KT Advisory Board.

Some contributors are staff members of the Pan American Health Organization. The authors alone are responsible for the views expressed in this publication. They do not necessarily represent the decisions or policies of funding or employing organizations.

\section{Funding}

COVID-END is supported by the Ontario Ministry of Health, Canada, the UK National Institute of Health Research, Evidence Synthesis Program, and individual donors through the Centre for Effective Altruism and Fidelity Charitable, U.S.A. and individual private donors. Jeremy Grimshaw holds a Canada Research Chair in Health Knowledge Transfer and Uptake and a CIHR Foundation grant 'Promoting Implementation of Evidence-based Care' (FDN-143269). John Lavis holds a Canada Research Chair in Evidence-Informed Health Systems.

\section{References}

1. Stewart R, El-Harakh A, Cherian SA, on behalf of the LMIC members of COVID-END. Evidence synthesis communities in low-income and middle-income countries and the COVID-19 response. Lancet 2020; October 20. https://doi.org/10.1016/S0140-6736(20)32141-3

2. Vandvik PO, Brandt L. Future of evidence ecosystem series: evidence ecosystems and learning health systems: why bother? Journal of Clinical Epidemiology 2020;123:166-170. https://doi.org/10.1016/j. jclinepi.2020.02.008 
3. Featherstone R, Last A, Becker L, Mavergames C. Rapid development of the Cochrane COVID-19 Study Register to support review production. In: Collaborating in response to COVID-19: editorial and methods initiatives across Cochrane. Cochrane Database of Systematic Reviews 2020;(12 Suppl 1). https://doi.org/10.1002/14651858.CD202002

4. Morley R, Crowe S, Haddaway N, Lytvyn L, Marshall C, Marshall $\mathrm{R}$, et al. Cochrane's COVID-19 consumer rapid response group. In: Collaborating in response to COVID-19: editorial and methods initiatives across Cochrane. Cochrane Database of Systematic Reviews 2020;(12 Suppl 1). https://doi.org/10.1002/14651858.CD202002
5. Kew K, De Haan S, Foxlee R, Marshall R. Prioritization during a pandemic: taking a strategic approach for a sustained response. In: Collaborating in response to COVID-19: editorial and methods initiatives across Cochrane. Cochrane Database of Systematic Reviews 2020;(12 Suppl 1). https://doi.org/10.1002/14651858.CD202002

6. Gough D, Davies P, Jamtvedt G, Langlois E, Littell J, Lotfi T, et al. Evidence synthesis international (ESI): position statement. Systematic Reviews. 2020;9(1):155. https://doi.org/10.1186/s13643-020-01415-5 


\title{
CEOsys: creating an ecosystem for COVID-19 evidence
}

\author{
Authors: Joerg J Meerpohl',2, Sebastian Voigt-Radloff ${ }^{1}$, Georg Rueschemeyer ${ }^{2}$, Felix Balzer ${ }^{3}$, Carina Benstoem ${ }^{4}$, \\ Harald Binder ${ }^{5}$, Martin Boeker ${ }^{5}$, Jacob Burns ${ }^{6,24}$, Ulrich Dirnagl ${ }^{7}$, Robin Featherstone ${ }^{8}$, Falk Fichtner ${ }^{9}$, Hajo \\ Grundmann ${ }^{10}$, Hartmut Hengel ${ }^{11}$, Volkhard Kempf ${ }^{12}$, Winfried Kern ${ }^{13}$, Peter Kranke ${ }^{14}$, Sven Laudi ${ }^{9}$, Klaus Lieb ${ }^{15}$, \\ Andy Maun ${ }^{16}$, Chris Mavergames ${ }^{8}$, Maria-Inti Metzendorf ${ }^{17}$, Monika Nothacker ${ }^{18}$, Christoph Schmaderer ${ }^{19}$, Christine \\ Schmucker ${ }^{1}$, Lukas Schwingshackl ${ }^{1}$, Nicole Skoetz ${ }^{20}$, Anke Steckelberg ${ }^{21}$, Miriam Stegemann ${ }^{22}$, Daniel Strech ${ }^{7}$, \\ Onnen Moerer ${ }^{23}$, Eva Rehfuess ${ }^{6,24}$, Claudia Spies ${ }^{3}$, on behalf of the CEOsys consortium
}

Participating groups: AWMF, Cochrane France, Cochrane Germany, Cochrane Central Executive, Institute for Science Journalism, Technical University (TU) Dortmund, 19 university medical centres

Corresponding Author: Joerg J Meerpohl: meerpohl@ifem.uni-freiburg.de

\section{Introduction and background}

Early in the course of the COVID-19 pandemic it became clear that the existing structures to produce evidence syntheses are extremely challenged to deliver the best possible evidence in the quickest possible way. While in the early days of the pandemic, there was simply very little direct evidence on COVID-19, there is now an evergrowing plethora of studies of very heterogeneous quality.

In May 2020, scientists of the Institute for Evidence in Medicine at the Medical Centre - University of Freiburg and Cochrane Germany responded to a funding call by the German Federal Ministry of Education and Research (BMBF) to establish a network of German university medical centers and strengthen their research activities on COVID-19. The aim of our application was the creation of an evidence ecosystem for COVID-19 research, a central reservoir of 'preprocessed' study data from Germany and beyond.[1,2,3] The project evolved during the review process to now involve a much larger consortium of scientists from 21 German university medical centers and several other partners, including the Association of the Scientific Medical Societies in Germany (AWMF), Cochrane Central Executive Team, and Cochrane France.

The idea behind the evidence ecosystem is to provide researchers and guideline developers in Germany and all over the world with a comprehensive, up-to-date source of curated data from clinical studies. Scientists involved with CEOsys will directly use this evidence repository to create a series of living evidence syntheses in the most pressing questions of the response to COVID-19.

The comprehensive approach of CEOsys goes beyond evidence synthesis: Based on the evidence assembled in the ecosystem, we

\footnotetext{
${ }^{1}$ Institute for Evidence in Medicine, Medical Center \& Faculty of Medicine, University of Freiburg, Freiburg, Germany; ${ }^{2}$ Cochrane Germany, Germany; ${ }^{3}$ Department of Anaesthesiology and Intensive Care Medicine (CCM, CVK), Charité-Universitätsmedizin Berlin, Corporate Member of Freie Universität Berlin, Humboldt Universität zu Berlin and Berlin Institute of Health, Germany; ${ }^{4}$ Department of Intensive Care Medicine and Intermediate Care, Medical Faculty, RWTH Aachen University, Germany; ${ }^{I n}$ stitute of Medical Biometry and Statistics, Medical Center \& Faculty of Medicine, University of Freiburg, Freiburg, Germany; ${ }^{6}$ Institute for Medical Information Processing, Biometry and Epidemiology, LMU Munich, Germany; ${ }^{7}$ Berlin Institute of Health (BIH), Charité Universitätsmedizin Berlin, Germany; ${ }^{8}$ Cochrane Central Executive; ${ }^{9}$ Department of Anesthesiology and Intensive Care Medicine, University of Leipzig Medical Center, Germany; ${ }^{10}$ Institute for Infection Prevention and Hospital Epidemiology, Medical Center \& Faculty of Medicine, University of Freiburg, Freiburg, Germany; ${ }^{11}$ Institute of Virology, Medical Center \& Faculty of Medicine, University of Freiburg, Freiburg, Germany; ${ }^{12}$ Institute of Medical Microbiology and Infection Control, Goethe University Hospital, Germany; ${ }^{13}$ Division of Infectious Diseases, Medical Center \& Faculty of Medicine, University of Freiburg, Freiburg, Germany; ${ }^{14}$ Department of Anaesthesia and Critical Care, University of Würzburg, Germany; ${ }^{15}$ Department of Psychiatry and Psychotherapy, University Medical Center Mainz and and Leibniz Institute for Resilience Research Mainz, Germany; ${ }^{16}$ Institute of General Practice, Medical Center \& Faculty of Medicine, University of Freiburg, Freiburg, Germany; ${ }^{17}$ Cochrane Metabolic and Endocrine Disorders Group, Institute of General Practice, Heinrich-Heine-University Düsseldorf, Germany; ${ }^{18}$ Institute for Medical Knowledge Management (AWMF-IMWi), AWMF office Berlin, Germany; ${ }^{19}$ Department of Nephrology, Technical University of Munich, School of Medicine, Klinikum rechts der Isar, Germany; ${ }^{20}$ Evidence-based Oncology, Department I of Internal Medicine and Center for Integrated Oncology Aachen Bonn Cologne Duesseldorf, Faculty of Medicine and University Hospital Cologne, University of Cologne, Germany; ${ }^{21}$ Institute for Health and Nursing Science, Martin Luther University Halle-Wittenberg, Halle (Saale), Germany; 22 Department of Infectious Diseases and Respiratory Medicine, Charité - Universitätsmedizin Berlin, Corporate Member of Freie Universität Berlin, Humboldt-Universität zu Berlin, and Berlin Institute of Health, Germany; ${ }^{23}$ Department for Anesthesiology, Göttingen Medical Center, Georg-August-University of Göttingen, Germany; ${ }^{24}$ Pettenkofer School of Public Health, Munich, Germany
} 


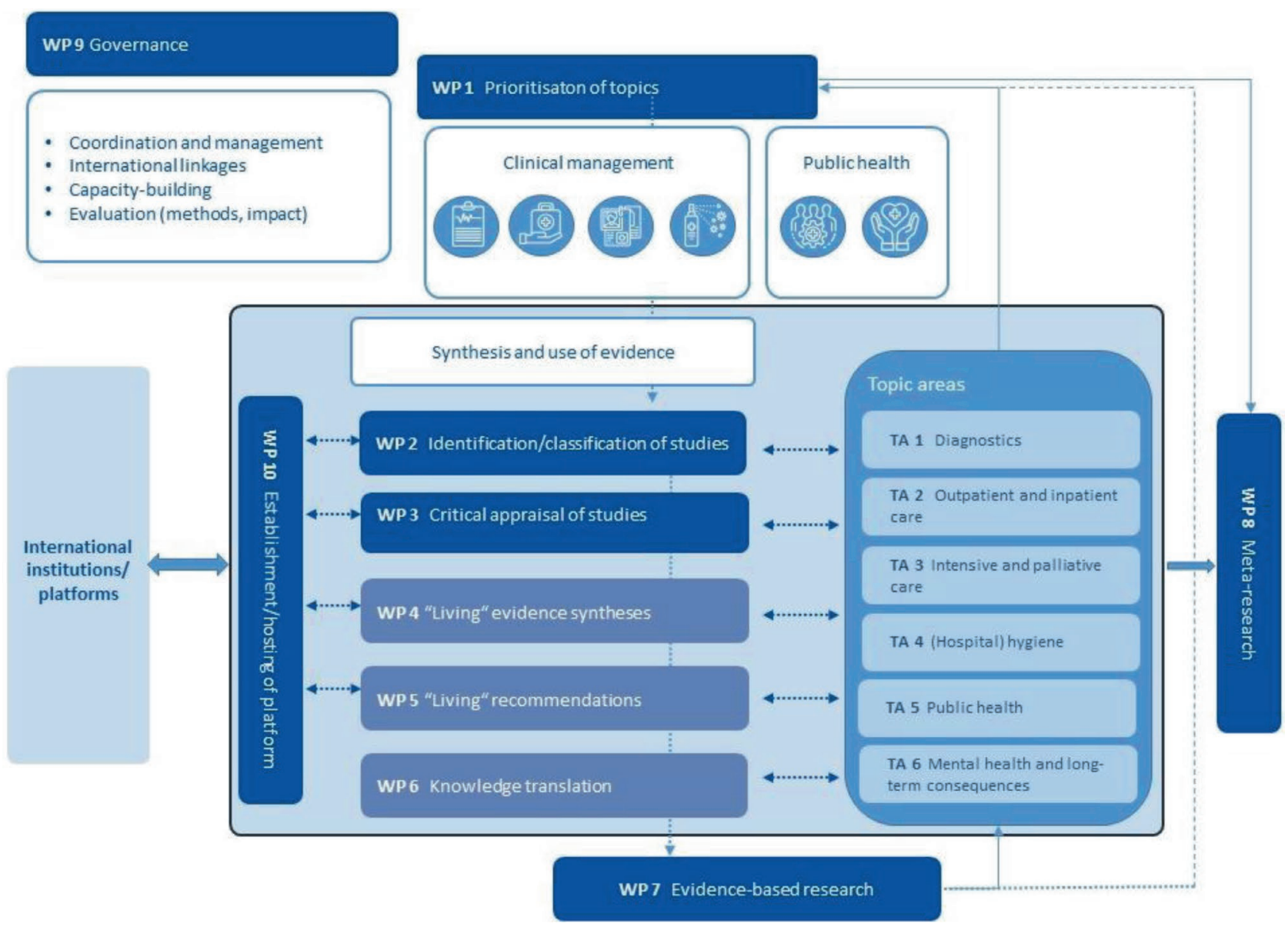

Figure 1. Structure of the CEOsys 10 work packages and six topical areas

will create living recommendations to inform clinical and public health practice. The recommendations will be developed together with representative, interdisciplinary guideline groups of the AWMF Task Force COVID-19 guidelines. Finally, we will communicate results to all relevant stakeholders, from patients and clinicians to scientists, policy makers and research funding agencies. To avoid duplication of effort, the project leads are in contact with a number of international projects aiming to provide better evidence about COVID-19. There will be direct links and integration with the Cochrane COVID-19 Study Register (covid-19.cochrane.org)[4] and the project COVID-NMA (covid-nma.com), led by Cochrane France. [5] CEOsys' activities have already started; funding was officially approved by the BMBF in early October 2020.

\section{Key activities and strategies}

The sheer size and number of collaborating institutions and scientists in CEOsys required a robust structural framework. The project is divided into 10 work packages and six topical areas (see Figure 1). The work packages provide a structure to the workflow, from prioritization of topics and all the steps of evidence synthesis and dissemination to governance. The topical areas represent scientists and clinicians working in six key areas of COVID-19 research.
1. Diagnostics

2. Outpatient and inpatient care

3. Intensive and palliative care

4. Hospital hygiene

5. Public health

Mental health and long-term consequences

The expert groups in CEOsys will continually and systematically identify scientific studies on COVID-19 using international databases, including the Cochrane COVID-19 Study Register.[4]

Our fast-acting analysis teams will evaluate new study results and produce continually updated 'living evidence syntheses'. These are transformed into up-to-date recommendations for action and into information that is then made available to the specific stakeholders (e.g. clinicians, patients, decision-makers) by a team of knowledge translation experts. With its wide network of experts from science and medical practice, CEOsys will establish communication channels with patients, clinicians, decision makers and the general public that also enables regular feedback. Furthermore, the network will enable standardized, transparent prioritization of research questions, identification of evidence gaps, and a 
co-ordinated research roadmap for the initiation of large-scale clinical studies on the most urgent issues.

The unique idea behind CEOsys is to go beyond the classical approach of an evidence synthesis that starts off with a specific (and often narrow) research question and then searches for the relevant literature. Instead, CEOsys will strive to collect and evaluate all scientific literature published on COVID-19 and relating to the above-described topical areas in an evidence ecosystem. We call it a 'living system' because new data are constantly being added and evaluated. We keep this ecosystem ready, so that when new questions appear, all the data are immediately available - ordered and appraised.

\section{Outcomes and impact of activities}

CEOsys will be a powerful, fast and interdisciplinary network of experts that aims critically to examine new scientific findings on COVID-19 pandemic management for relevance in a quality-assured, financially-independent manner and in the quickest possible way. The ultimate goal is to provide clinicians and decision-makers with recommendations for action and thus make sure that decisions in the times of COVID-19 are based on the best possible evidence. We are extremely grateful for the trust placed in us by the BMBF and for the spirit of collaboration shown by all involved.

\section{Lessons for the future: sustainability and transferability}

The COVID-19 pandemic has challenged the evidence synthesis community. One of the problems that is becoming increasingly obvious, particularly in Germany, is a lack of co-ordination in clinical and public health research, resulting in a plethora of small, underpowered studies, lacking comparability. One lesson from this for Cochrane and the research community at large is that we need to advocate even harder for research that is better organized and co-ordinated. One of CEOsys' key visions is to design and test a blueprint for how to rapidly produce, synthesize and communicate evidence in future pandemics or health emergencies. CEOsys will create a methodological toolkit and platform that can be reactivated during a future crisis.

\section{Additional resources}

- AWMF platform for COVID-19 guidelines: awmf.org/die-awmf/awmfaktuell/aktuelle-leitlinien-und-informationen-zu-covid-19/covid-19leitlinien.html

- CEOsys: covid-evidenz.de

- Network for University Medicine: netzwerk-universitaetsmedizin.de

- Cochrane Germany COVID-19 information: cochrane.de/de/covid-19evidenz-von-cochrane-und-andere-ressourcen

\section{Acknowledgements}

Full membership of the CEOsys project can be found on the CEOsys website: www.covid-evidenz.de
We thank the many contributors to CEOsys from the following 19 German university medical centers, all part of the German Network of University Medicine: Aachen, Berlin, Bonn, Düsseldorf, Frankfurt, Freiburg, Göttingen, Greifswald, Halle, Jena, Köln, Leipzig, Lübeck, Mainz, Marburg, München, Münster, Ulm, Würzburg.

We also thank our external partners: AWMF, Marburg; Cochrane France; Cochrane Germany; Cochrane International Mobility; and Institute for Science Journalism, TU Dortmund.

We are grateful for the funding of CEOsys by the German Federal Ministry for Education and Research (BMBF) through the Network for University Medicine.

\section{Declarations of interest}

The CEOsys project is funded by the German Federal Ministry for Education and Research as part of the Network for University Medicine. Joerg J Meerpohl is Director, Sebastian Voigt-Radloff is a Researcher, and Georg Rueschemeyer is an employee of Cochrane Germany. Eva Rehfuess is an Editor at Cochrane Public Health. Nicole Skoetz is Senior Editor of Cochrane Cancer. Robin Featherstone and Chris Mavergames are Cochrane Central Executive staff. The authors declare that they have no competing interests.

\section{Funding}

The CEOsys project is funded by the German Federal Ministry for Education and Research as part of the Network for University Medicine (Grant number 01KX2021). The Cochrane Germany Foundation receives funding from the German Federal Ministry of Health.

\section{References}

1. Boutron I, Créquit P, Williams H, Meerpohl J, Craig JC, Ravaud P. Future of evidence ecosystem series: 1 . Introduction Evidence synthesis ecosystem needs dramatic change. Journal of Clinical Epidemiology 2020;123:135-42. https://doi.org/10.1016/j.jclinepi.2020.01.024

2. Créquit P, Boutron I, Meerpohl J, Williams HC, Craig J, Ravaud P. Future of evidence ecosystem series: 2. current opportunities and need for better tools and methods. Journal of Clinical Epidemiology 2020;123:143-52. https://doi.org/10.1016/j.jclinepi.2020.01.023

3. Ravaud P, Créquit P, Williams HC, Meerpohl J, Craig JC, Boutron I. Future of evidence ecosystem series: 3 . From an evidence synthesis ecosystem to an evidence ecosystem. Journal of Clinical Epidemiology 2020;123:153-61. https://doi.org/10.1016/j.jclinepi.2020.01.027

4. Featherstone R, Last A, Becker L, Mavergames C. Rapid development of the Cochrane COVID-19 Study Register to support review production. In: Collaborating in response to COVID-19: editorial and methods initiatives across Cochrane. Cochrane Database of Systematic Reviews 2020;(12 Suppl 1). https://doi.org/10.1002/14651858.CD202002

5. Boutron I, Tovey D, De Nale L, Chaimani A, Devane D, Meerpohl JJ, et al, for the COVID-NMA Consortium. COVID-NMA: a collaborative COVID-19 living evidence project. In: Collaborating in response to COVID-19: editorial and methods initiatives across Cochrane. Cochrane Database of Systematic Reviews 2020;(12 Suppl 1). https://doi. org/10.1002/14651858.CD202002 


\title{
COVID-19 SHORT REPORT
}

\section{Prioritization during a pandemic: taking a strategic approach for a sustained response}

\author{
Authors: Kayleigh Kew ${ }^{1}$, Sylvia de Haan ${ }^{2}$, Ruth Foxlee ${ }^{1}$, Rachel Marshall ${ }^{1}$ \\ Participating groups: Cochrane Central Executive Team, Cochrane Review Group Networks, Cochrane Fields, \\ Cochrane Geographic Groups, Cochrane COVID-19 Working Group, Cochrane Library Editorial Board, Cochrane \\ Methods Groups, Cochrane Consumer Network
}

Corresponding Author: Kayleigh Kew: kkew@cochrane.org

\section{Introduction and background}

The proliferation of research during disease outbreaks often mirrors the speed at which the infection itself spreads across the globe. Advice changes and new uncertainties often arise faster than governments and healthcare staff can keep up with, and strategic action is needed to inform research priorities.[1] Cochrane was well placed to respond to the COVID-19 pandemic due to its vast network of contributors, connection to key partners, and reputation for highquality evidence syntheses, but new approaches were needed to meet time pressures of the pandemic.

Cochrane adapted its systems and workflows to enable fast-track production and publication through collaborative working between Cochrane Review Groups (CRGs) and the Cochrane Editorial Service (community.cochrane.org/review-production/production-resources/ cochranes-central-editorial-service).[2] The adapted fast-track processes required significant resources and co-ordinated input from Cochrane Groups and experienced, well-resourced author teams. A strategic approach to prioritization was required to make the best use of resources and expertise without duplicating effort.

\section{Key activities and strategies}

Members of the Cochrane Central Executive Team (CET) conducted a prioritization process with a range of stakeholders to identify where Cochrane should direct its efforts in the immediate and longer term. The process followed three stages.

1. Engage with end users and partner organizations including the World Health Organization to collect priority questions.

2. Categorize the questions to prioritize a limited number of key reviews, which Cochrane would rapidly produce and keep up to date.

3. Create a flexible priority framework to guide Cochrane's sustained response to the pandemic.
More than 250 questions were submitted by research organizations, policymakers, frontline workers and researchers, which we had to collate and categorize before prioritization could take place. With no standard taxonomy for COVID-19, we created an iterative classification system to group related questions. We started with question type (diagnosis, treatment, prognosis, prevention, epidemiology, etc) and later incorporated keyword tags to describe population, intervention, outcome and setting. The categorization process identified groups of questions that needed to be considered together and allowed prioritization to occur in batches with experts, external stakeholders and Cochrane Groups who were asked to consider:

1. whether the answer would help inform an urgent clinical or policy decision;

2. whether there was overlap or duplication with other submitted questions;

3. whether relevant Cochrane or non-Cochrane Reviews were already published or underway;

4. the suitability of the question for Cochrane Review methods.

The COVID Rapid Reviews website and Question Bank were launched early in the process as a resource for the research community while prioritization took place (covidreviews.cochrane. org).[3] The site indicated which questions were being pursued by Cochrane, which were known to be underway with our partners, and which were open to new author teams.

\section{Outcomes and impact of activities}

The main outcome of our prioritization work is a framework, created by Cochrane's Editor in Chief, Karla Soares-Weiser and Cochrane's COVID-19 Working Group, that shows which reviews are eligible for additional support and which are the key topic areas for the fast-track editorial process. The priority framework (covidreviews.cochrane.org/sites/covidreviews.cochrane.org/files/

${ }^{1}$ Cochrane Central Executive, UK; ${ }^{2}$ Cochrane Central Executive, The Netherlands 
public/uploads/covid-19_priorities_13_july_2020.pdf) is used to guide decision-making, with input from CRG staff and the Cochrane Editorial Board, as new questions and proposals are submitted. The three priority areas are aligned with the taxonomy of the 'COVID-19 Evidence Network to support Decision-making' (COVID-END; www. mcmasterforum.org/networks/covid-end), of which Cochrane is a leading member.[4,5]

1. Clinical management: including questions about screening and testing, drug treatment, critical care, prophylaxis, prognosis and the clinical management of pandemic related impacts on health

2. Public health measures: including questions about the prevention of infection, personal protection and the need to support healthcare workers

3. Economic and social responses: including questions about the social determinants of health and their impact on health outcomes, and the impact of COVID-19 on food poverty.

Each of these areas is being addressed in a range of ways including through Cochrane Rapid Reviews, [6] Cochrane living systematic reviews,[7] priority updates, and network meta-analyses. In August 2020, the Question Bank was relaunched as a Review Bank, which gives readers an overview of Cochrane's full body of COVID-19 priority reviews (covidreviews.cochrane.org/search/site). Overlapping and duplicate questions, and those unsuitable for systematic methods, have been gradually removed as prioritization work progressed, thus refining and clarifying Cochrane's COVID-19 priorities and publicizing areas where new proposals are being invited.

\section{Lessons for the future: sustainability and transferability}

A key learning from the COVID-19 response has been the importance of having a clear scope and setting up efficient triage processes, which help identify question overlap and feasibility. We developed a streamlined review proposal process for authors based on a rapid review protocol template, which is submitted with an accompanying cover letter. This approach reduced the time to decision and improved the consistency and transparency of decision-making. A high volume of submissions has been processed because key information about the priority area, related reviews, anticipated level of evidence, team expertise, and the proposed scope and methods are elicited in a consistent way.

Topic refinement by an information specialist has also proven hugely useful during prioritization to prevent overlap and develop well-formulated, clinically relevant and feasible review questions. [8] Existing evidence is flagged for authors to consider when developing a protocol and suggestions for how the question might be broadened or narrowed are included. Topic refinement, with the priority framework and the streamlined proposal process, has helped the prioritization team to seek appropriate clinical and methodological input and reach consistent decisions as priorities have evolved over time. This agility was essential as decisionmakers' focus moved from immediate public health questions to the longer-term effects from measures taken to manage the pandemic. A key area for future development is to extend scoping and triage criteria to inform decisions about the suitability of review questions and methods for rapid, living, or standard approaches.

The most important challenge in the COVID-19 response has been acting quickly enough to get answers to decision makers when they need them, but taking sufficient time to ensure that those answers are relevant and reliable. Prioritization has enabled us to apply Cochrane's trademark standards for prespecification, careful question development and quality assurance efficiently in a tumultuous time. Cochrane's collaborative working models have underpinned our COVID-19 response and provide a firm foundation for defining responsibilities and workflows as our current COVID-19 review portfolio is delivered, and future public health priorities are identified and addressed.

\section{Additional resources}

- Cochrane COVID-19 Reviews website: covidreviews.cochrane.org

- Cochrane's Priority Framework: covidreviews.cochrane.org/ sites/covidreviews.cochrane.org/files/public/uploads/covid-19_ priorities_13_july_2020.pdf

- The Cochrane Rapid Review Template is available to download from covidreviews.cochrane.org/resources

- The streamlined review proposal process for COVID-19 Rapid Reviews is described at covidreviews.cochrane.org/process

\section{Acknowledgements}

Karen Head, Robin Featherstone, Froeks Kamminga, Toby Lasserson, Karla Soares-Weiser, and the information specialists who conducted topic refinements (Ruth Foxlee, Irma Klerings, Douglas Salzwedel, and Liz Stovold) were all fundamental to the success of the work described. The publication of priority COVID-19 reviews has only been possible due to the significant and sustained work of review authors and all those who have contributed to their fast-track quality assurance and publication: Helen Wakeford and everyone in the Cochrane Editorial Service; Cochrane Review Group staff and Network Associate Editors; the Cochrane Public Health Hub; the Methods Support Unit; Methods Groups; peer reviewers and consumers; copy editors; the Cochrane Editorial Board; and Cochrane publishing and knowledge translation teams.

\section{Declarations of interest}

All authors are employed by, and receive a salary from, Cochrane as part of its Central Executive team. KK, RF and RM are members of the Editorial and Methods Department and $\mathrm{SdH}$ is a member of the Chief Executive Officer's Office. The authors declare that they have no other competing interests.

\section{Funding}

None 


\section{References}

1. Sigfrid L, Moore C, Salam AP, Maayan N, Hamel C, Garritty C, et al. A rapid research needs appraisal methodology to identify evidence gaps to inform clinical research priorities in response to outbreaks-results from the Lassa fever pilot. BMC Medicine 2019;17:107. https://doi. org/10.1186/s12916-019-1338-1

2. Wakeford H, Dooley C, Stephani A-M, Marshall R, Featherstone R, Walshe D, et al. The Central Editorial Service: a collaborative editorial process for publishing high-priority Cochrane reviews. In: Collaborating in response to COVID-19: editorial and methods initiatives across Cochrane. Cochrane Database of Systematic Reviews 2020;(12 Suppl 1). https://doi.org/10.1002/14651858.CD202002

3. Cochrane. Cochrane COVID Reviews. covidreviews.cochrane.org (accessed 3 September 2020).

4. McMaster Health Forum. COVID-19 Evidence Network to support Decision-Making. mcmasterforum.org/networks/covid-end (accessed 3 September 2020).

5. Grimshaw JM, Tovey DI, Lavis JN, on behalf of COVID-END. COVIDEND: an international network to better co-ordinate and maximize the impact of the global evidence synthesis and guidance response to COVID-19. In: Collaborating in response to COVID-19: editorial and methods initiatives across Cochrane. Cochrane Database of Systematic Reviews 2020;(12 Suppl 1). https://doi.org/10.1002/14651858.CD202002

6. Garritty C, Gartlehner G, Nussbaumer-Streit B, King VJ, Kamel C, Stevens A, et al. Rapid review methods guidance aids in Cochrane's quick response to the COVID-19 crisis. In: Collaborating in response to COVID-19: editorial and methods initiatives across Cochrane. Cochrane Database of Systematic Reviews 2020;(12 Suppl 1). https://doi. org/10.1002/14651858.CD202002

7. Turner T, McDonald S. Cochrane COVID-19 living systematic reviews: why, how, and what has been done? In: Collaborating in response to COVID-19: editorial and methods initiatives across Cochrane. Cochrane Database of Systematic Reviews 2020;(12 Suppl 1). https://doi. org/10.1002/14651858.CD202002

8. Thomas J, Kneale D, McKenzie JE, Brennan SE, Bhaumik S. Chapter 2: Determining the scope of the review and the questions it will address. In: Higgins JP, Thomas J, Chandler J, Cumpston M, Li T, Page MJ, Welch VA (editors). Cochrane Handbook for Systematic Reviews of Interventions version 6.0 (updated July 2019). Cochrane, 2019. Available from training.cochrane.org/handbook. 


\section{Developing a core outcome set for COVID-19 prevention interventions: the COS COVID-P study}

Authors: Kerry Avery ${ }^{1}$, Rhiannon Macefield ${ }^{1}$, Sarah Gorst ${ }^{2}$, Nicola Harman ${ }^{2}$, Susanna Dodd ${ }^{2}$, Mike Clarke ${ }^{3,4}$, Paula Williamson ${ }^{2 \star}$, Jane Blazeby ${ }^{1 *}$, on behalf of the COS COVID-P Study Steering Committee

Participating groups: COMET Initiative, Cochrane Methodology Review Group, Cochrane Epilepsy, Cochrane Effective Practice and Organisation of Care, Cochrane Kidney and Transplant, Cochrane China, Cochrane Stroke, Cochrane Consumer Network, Cochrane Central Executive Team, COVID-evidence, ISARIC, Evidence Aid

Corresponding Author: Paula Williamson:p.r.williamson@liverpool.ac.uk

\section{Introduction and background}

Interventions that minimize the spread of SARS-CoV-2 are essential to reduce COVID-19 morbidity and mortality. Various methods are being implemented worldwide, including vaccines and drugs for prophylaxis (Figure 1). Public health safety interventions such as social distancing, school closure and behavioural interventions such as hand washing and use of personal protective equipment (PPE), are increasing all the time. Research into the effectiveness of these interventions is accumulating, but evidence synthesis on the prevention of COVID-19 is unnecessarily difficult because of inconsistencies in how outcomes are measured.[1-3] The recent rapid update of the Cochrane review of PPE highlights this, with the review authors concluding that consensus around outcome assessment is urgently needed.[1]

One solution would be a core outcome set (COS), an agreed standardised set of outcomes that would be measured and reported, as a minimum, in all studies of interventions to prevent SARS-CoV-2 infection.[4] COS focusing on treatment of people with COVID-19 are available,[5-8] and a 'meta-COS' for hospitalised adults with confirmed or suspected COVID-19 has been agreed with two domains: mortality and respiratory support. $[9,10]$ A COS for COVID-19 prevention interventions is overdue.

The COS COVID-P study aims to fill this gap, with a COS that will include a minimum set of outcomes for prevention studies worldwide.[4] This may involve identification of core outcome domains relevant to all types of COVID-19 prevention interventions or modules for specific interventions or settings, or both. Widespread implementation of the COS will improve the quality and consistency of study reporting, enhancing the value of evidence synthesis, facilitating application of findings and reducing waste.

\section{Key activities and strategies}

This rapid project began with registration on the COMET database on 3 July 2020,[4] followed by the first step of examining outcomes measured in COVID-19 prevention intervention studies in the Cochrane COVID-19 study register[11,12] and COVID-Evidence repository.[13] This revealed considerable heterogeneity in outcome measurement, even within the same intervention type or setting, further highlighting the urgent need for the COS.

The second step focused on defining the COS scope. We needed agreement on whether it was possible to develop a COS for prevention interventions that targeted stopping someone from becoming infected or an infected person from transmitting the disease. We brought together key international stakeholders in three online workshops, with a total of 71 participants: 25 patient/ public representatives, 16 researchers, 12 clinician researchers, $10 \mathrm{COS}$ methodologists, four systematic reviewers, two guideline developers, and two regulatory agency representatives. They discussed whether a COS was needed; should the focus be solely on prevention of infection, or include transmission, feasibility of developing a COS for all interventions, grouping of interventions, and whether a single COS or modular approach was recommended.

We fed back findings to the steering committee meeting within two weeks, with 22 international members voting anonymously in the online meeting. 


\section{Pharmacological Interventions}

\section{Behavioural, Environmental, Social and Systems Interventions}
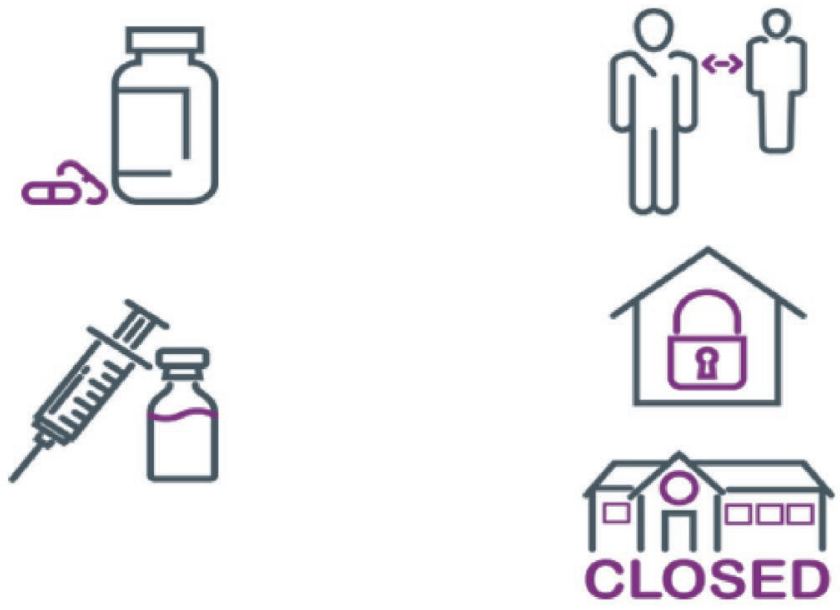

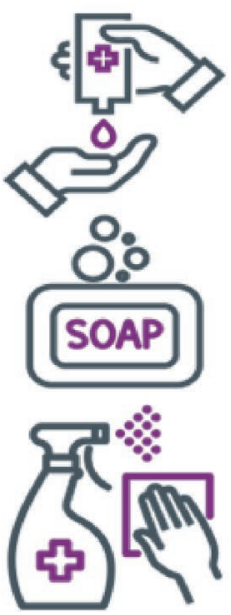

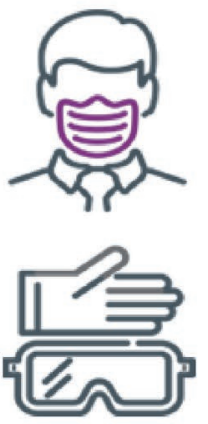

Figure 1. Categories of COVID-19 prevention interventions

\section{Outcomes and impact of activities}

The steering committee unanimously supported development of the COS. After initial voting was split between a 'core' COS relevant to studies of prevention or transmission of infection, or both (54\%) or one for prevention only (46\%), they agreed initially to limit the scope to prevention of infection, given the developing understanding of COVID-19 and the rapidly shifting landscape of COVID-19 research.

Members also reached agreement on inclusion of two outcome domains (infection and intervention-specific harms), but the majority did not support including a measure of severity of disease (58\% of members against). Most (91\%) members supported the idea of a core COS relevant to any intervention type.

\section{Lessons for the future: sustainability and transferability}

Our work to date has identified key issues for designing COVID-19 prevention studies, including that SARS-CoV-2 infection is an essential outcome to measure. However, there has been much discussion of challenges around how to measure infection. Entries in the Cochrane register show that infection is measured in different ways, including through laboratory tests, observation of symptoms or a combination of both.[11] Other issues include the lack of a single, standardized laboratory test or agreement on which symptoms define a positive infection.[14] The knowledge base for these is still fluid as new evidence emerges, and consideration needs to be given to testing feasibility and capacity, particularly to ensure global uptake of the COS.
Understanding of the mechanisms by which COVID-19 prevention interventions may work is also developing. There is uncertainty around which prevention interventions prevent acquiring SARSCoV-2, which attenuate the effect of the disease, and which prevent infection by reducing transmission or influencing behaviour. This requires continuing engagement with specialist disease prevention and clinical experts, to inform how researchers may optimally measure infection and, where relevant, develop interventionspecific COS modules.

Standard methods for developing COS are well established. [15] These typically include systematic reviews and stakeholder interviews to identify potentially relevant outcomes for a Delphi survey to prioritise outcomes for the COS. Such steps have taken months or years in other COS but the speed with which evaluations of COVID-19 prevention interventions are being developed and implemented requires an expedited approach for COS COVID-P. [11] This brings challenges, not least difficulties with gaining understanding of a novel disease in a short space of time, which is crucial to achieve clarity around the scope of the COS. Rapid identification of and collaboration with key stakeholders (who are busy contributing in other ways to the COVID-19 research effort) has been challenging, but the support and engagement from contributors worldwide has been both positive and fruitful.

We are on the way, therefore, to developing guidance for those designing COVID-19 prevention studies around how infection may be optimally defined and measured so that all these studies can make the maximum contribution to meeting this global challenge. 
We need to consider how to increase the uptake of available cos in relevant systematic reviews and trials, and ensure researchers are considering them at the correct stage of the research process. It is important to note that the Cochrane Rapid Review protocol template (covidreviews.cochrane.org/resources) [16] and the Cochrane Handbook for Systematic Reviews of Interventions[17] advise authors to check for COS. Several trial funders are also recommending that applicants consider COS for COVID-19 studies (see comet-initiative.org/COSEndorsement/TrialFunders).

\section{Additional resources}

COS COVID-P study registration: comet-initiative.org/Studies/ Details/1594

\section{Acknowledgements}

In addition to article authors, members of the COS COVID-P Study Steering Committee include: Elie Akl (American University of Beirut, Lebanon); Aneel Bhangu (University of Birmingham, UK); Deborah Caldwell (University of Bristol, UK); Jonathan Craig (Flinders University, Australia); Liang Du (Cochrane China); Adam Finn (University of Bristol, UK); Ella Flemyng (Cochrane, UK); Atle Fretheim (Norwegian Institute of Public Health, Norway); Claire Goodman (University of Hertfordshire, UK); Lars Hemkens (University of Basel, Switzerland); Tammy Hoffman (Bond University, Australia); John Ioannidis (Stanford University, USA); Martin Knapp (London School of Economics, UK); David Lalloo (Liverpool School of Tropical Medicine, UK); Susan Michie (UCL, UK); Richard Morley (Cochrane, UK); Calum Semple (University of Liverpool, UK); Maureen Smith (Cochrane Consumer Network, Canada); Allison Tong (University of Sydney, Australia); Sean Tunis (Center for Evaluation of Value and Risk in Health, USA); Junhua Zhang (Tianjin University of Traditional Chinese Medicine, China).

We would also like to thank those stakeholders who took part in the online scoping workshops.

\section{Declarations of interest}

MC, PW and JB are members of the COMET Management Group. Ella Flemyng, member of the COS COVID-P Study Steering Committee, is employed by Cochrane and co-ordinated the COVID-19 supplement to the Cochrane Database of Systematic Reviews but was not involved in any editorial handling or decisions for this short report.

\section{Funding}

This study is supported by National Institute for Health Research (NIHR) Senior Investigator Awards (NF-SI_0513-10025 and NIHR200268), the National Institute for Health Research (NIHR) Biomedical Research Centre (BRC) at University Hospitals Bristol and Weston NHS Foundation Trust and the University of Bristol (BRC-1215-20011) and the Royal College of Surgeons of England Bristol Surgical Trials Centre. The views expressed in this publication are those of the authors and not necessarily those of the NIHR, the Department of Health and Social Care or the Royal College of Surgeons of England. JB and PW are NIHR Senior Investigators.

\section{References}

1. Verbeek JH, Rajamaki B, ljaz S, Sauni R, Toomey E, Blackwood B, et al. Personal protective equipment for preventing highly infectious diseases due to exposure to contaminated body fluids in healthcare staff. Cochrane Database of Systematic Reviews 2020;(5):CD011621. https:// doi.org/10.1002/14651858.CD011621.pub5

2. Chu DK, Akl EA, Duda S, Solo K, Yaacoub S, Schünemann HJ, et al. Physical distancing, face masks, and eye protection to prevent personto-person transmission of SARS-CoV-2 and COVID-19: a systematic review and meta-analysis. Lancet 2020;395(10242):1973-87. https://doi. org/10.1016/S0140-6736(20)31142-9

3. Nussbaumer-Streit B, Mayr V, Dobrescu A, Chapman A, Persad E, Klerings I, et al. Quarantine alone or in combination with other public health measures to control COVID-19: a rapid review. Cochrane Database of Systematic Reviews 2020;(9):CD013574. https://doi. org/10.1002/14651858.CD013574.pub2

4. Blazeby J, Williamson P. A core outcome set for studies evaluating public health, primary and secondary care interventions for prevention of COVID-19 transmission: the COS-COVID-P study. comet-initiative.org/ Studies/Details/1594

5. Qiu R, Zhao C, Liang T, Hao X, Huang Y, Zhang X, et al. Core outcome set for clinical trials of COVID-19 based on traditional Chinese and western medicine. Frontiers in Pharmacology 2020;11:781. https://doi. org/10.3389/fphar.2020.00781

6. Marshall JC, Murthy S, Diaz J, Adhikari NK, Angus DC, Arabi YM, et al. A minimal common outcome measure set for COVID-19 clinical research. Lancet Infectious Diseases 2020;20(8):e192-e7. https://doi.org/10.1016/ S1473-3099(20)30483-7

7. Jin X, Pang B, Zhang J, Liu Q, Yang Z, Feng J, et al. Core outcome set for clinical trials on coronavirus disease 2019 (COS-COVID). Engineering. 2020;March 18 https://doi.org/10.1016/j.eng.2020.03.002

8. Tong A, Elliott JH, Azevedo LC, Baumgart A, Bersten A, Cervantes L, et al. Core outcomes set for trials in people with coronavirus disease 2019. Critical Care Medicine 2020. 48(11):1622-35. https://doi.org/10.1097/ CCM.0000000000004585

9. COMET Initiative. Core outcome set developers' response to COVID-19. 7 July 2020. comet-initiative.org/Studies/Details/1538

10. COMET Initiative. The 'meta-COS' for research in COVID-19 hospitalised patients. 29 October 2020. https://www.comet-initiative. org/assets/downloads/COVID-19\%20meta\%20COS_Table\%20 1_29th\%200ctober\%202020.pdf.

11. Cochrane. Cochrane COVID-19 Study Register. covid-19.cochrane. org.

12. Featherstone R, Last A, Becker L, Mavergames C. Rapid development of the Cochrane COVID-19 Study Register to support review production. In: Collaborating in response to COVID-19: editorial and methods initiatives across Cochrane. Cochrane Database of Systematic Reviews 2020;(12 Suppl 1). https://doi.org/10.1002/14651858.CD202002 
13. COVID-evidence. covid-evidence.org.

14. Struyf T, Deeks JJ, Dinnes J, Takwoingi Y, Davenport C, Leeflang $M M$, et al. Signs and symptoms to determine if a patient presenting in primary care or hospital outpatient settings has COVID-19 disease. Cochrane Database of Systematic Reviews 2020;(7):CD013665. https:// doi.org/10.1002/14651858.CD013665

15. Williamson PR, Altman DG, Bagley H, Barnes KL, Blazeby JM, Brookes ST, et al. The COMET Handbook: version 1.0. Trials 2017;18(Suppl 3):280. https://doi.org/10.1186/s13063-017-1978-4

16. Garritty C, Gartlehner G, Nussbaumer-Streit B, King VJ, Kamel C, Stevens A, et al. Rapid review methods guidance aids in Cochrane's quick response to the COVID-19 crisis. In: Collaborating in response to COVID-19: editorial and methods initiatives across Cochrane. Cochrane Database of Systematic Reviews 2020;(12 Suppl 1). https://doi. org/10.1002/14651858.CD202002

17. McKenzie JE, Brennan SE, Ryan RE, Thomson HJ, Johnston RV, Thomas J. Chapter 3: Defining the criteria for including studies and how they will be grouped for the synthesis. In: Higgins JP, Thomas J, Chandler J, Cumpston M, Li T, Page MJ, et al (editors). Cochrane Handbook for Systematic Reviews of Interventions version 6.1 (updated September 2020). Cochrane, 2020. Available from www.training. cochrane.org/handbook 


\section{COVID-19 SHORT REPORT}

\section{Cochrane's COVID-19 consumer rapid response group}

Authors: Richard Morley ${ }^{1}$, Sally Crowe ${ }^{2}$, Neal Haddaway ${ }^{3}$, Lyubov Lytvyn ${ }^{4}$, Catherine Marshall ${ }^{5}$, Rachel Marshall ${ }^{1}$, Alex Pollock ${ }^{6}$, Maureen Smith ${ }^{7}$, Anneliese Synnot ${ }^{8}$, Oliver Willis ${ }^{1}$

Participating groups: Cochrane Consumer Network, Cochrane Consumer Network Executive, Cochrane Central Executive, Cochrane Australia, Cochrane UK, COVID-19 consumer rapid response group

Corresponding Author: Richard Morley: rmorley@cochrane.org

\section{Introduction and background}

As a result of the coronavirus (COVID-19) pandemic, Cochrane prioritized work on the production of rapid reviews of evidence to inform the most urgent health decisions. The challenge was to create a process, develop resources, and recruit people with lived experience quickly, so that healthcare consumer perspectives would be a part of the rapid review process, in line with relevant Cochrane policies.[1,2]

A COVID-19 consumer rapid response task group was formed in early April 2020, made up from 10 stakeholders experienced in healthcare consumer involvement in Cochrane evidence production (consumers.cochrane.org/learning/covid-19-consumer-rapidresponse-group-resources). The aim of this group was to advise on the formation of an involvement process, and the creation of resources for researchers, editors and healthcare consumers.

\section{Key activities and strategies}

Following their formation, the task group considered several key areas.

- Defining the range of people whose voluntary contributions would be sought, namely people who: are ill or have recovered from COVID-19; are at high risk of COVID-19 infection (such as immunosuppressed people, people living in high-risk areas, people working with sick people); who have experience caring for someone with COVID-19 as an informal caregiver; are family members or cohabitants of people who have experienced COVID-19; have lived experience of other conditions (e.g. mental health, cancer) that are impacted by COVID-19; who do not have direct lived experience related to COVID-19 but are interested in contributing a healthcare consumer perspective to reviews and guidelines.
- Creating a process for potential volunteers to register an interest online.

- Defining the range of ways consumer contributions could be made to a rapid review process. These included working as part of the author team; consumer peer review; supporting the dissemination of Cochrane evidence; sharing evidence and information about COVID-19 on social media; and peer support for new consumer volunteers.

- Developing a range of resources for healthcare consumers, researchers and editors, to support the involvement of healthcare consumers. These included a welcome pack for volunteers; one-page guidance, short recorded videos; a web page for consumers, author teams and editors.

- Producing a series of promotional activities including two community blogs, social media posts and mailing the Cochrane Consumer Network's 1750 members to recruit members of the consumer rapid response group.

- Recruiting experienced consumers who could support new consumers members of the group.

Throughout this work the task group drew on established networks including the Cochrane Consumer Network,[3] and its work was informed by the work of the ACTIVE project, which established a framework for involvement in Cochrane evidence production,[4] and existing resources that had been developed over previous years.[5]

\section{Outcomes and impact of activities}

108 healthcare consumers from 28 countries have volunteered to be a part of the consumer rapid response group. People in the group have a range of lived experience, including seven people who have recovered from COVID-19. To date, 20 reviews have involved members of the COVID-19 rapid response consumer group. Sixteen 
have involved consumers as peer reviewers, with four having included consumers in their author teams.

Other activity included three opportunities for consumers to share information on social media. In addition, we have supported non-Cochrane activities including an Australian guideline development team (via Cochrane Australia).

Further rapid reviews are in development at the time of writing this report and we anticipate significant activity in the future.

\section{Lessons for the future: sustainability and transferability}

The task group is presently developing an approach to assess the impact of the work and gather feedback from all participants. It will include surveys of all participants in the process, using the ACTIVE framework, [4] to determine in individual reviews: what happened (methods and approach); the stages, level and impact of involvement; and participants' reflections on the process. We hope to be able to report more about this approach in due course.

There were significant challenges in undertaking this activity. These included the speed at which it was required to work to react to both the pandemic and Cochrane's COVID-19 response; the production of targeted resources that were needed for consumers and researchers to participate in a rapid response process; managing expectations so that we did not recruit more consumers than could meaningfully take part given the general willingness of consumers to volunteer; establishing systems and processes that would facilitate involvement; and communicating and co-ordinating the consumer response with other teams in Cochrane.

So far, consumer input has been largely confined to peer review, whereas the Statement of Principles for Consumer Involvement asserts Cochrane's view that "...the benefits of consumer involvement are best realised when consumers contribute throughout the process of production and dissemination of research." [2] More outreach to authors and researchers needs to be done to involve consumers in these areas.

Research on the pandemic and its consequences will be with us for some time. Cochrane's response to the global pandemic will move on from rapid reviews, to living systematic reviews (systematic reviews that are continually updated, incorporating relevant new evidence as it becomes available) and standard Cochrane reviews. A structured approach to enabling consumer involvement will continue to be vital.

\section{Additional resources}

COVID-19 consumer rapid response group resources: consumers. cochrane.org/learning/covid-19-consumer-rapid-response-groupresources

\section{Acknowledgements}

We would like to thank all of the consumers who have signed up to the COVID-19 consumer rapid response group or contributed to Cochrane Reviews so far. The founding members of the COVID-19 consumer rapid response task group are Sally Crowe, Neil Haddaway, Lyuba Lytvyn, Catherine Marshall, Rachel Marshall, Richard Morley, Alex Pollock, Maureen Smith, Annie Synnott and Oliver Willis (all authors of this report). We also acknowledge the ACTIVE project,[4] which established a framework for involvement in Cochrane evidence production. We also would like to acknowledge the work of Cochrane UK and Selena Ryan-Vig.

\section{Declarations of interest}

Richard Morley, Rachel Marshall and Oliver Willis are employees of Cochrane. Catherine Marshall is a member of the Cochrane Governing Board. Maureen Smith is a Chair of the Cochrane Consumer Executive. Annelise Synnott works with the Cochrane Consumer and Engagement Review Group. Alex Pollock is joint Co-ordinating Editor of Cochrane Stroke Review Group. Lyuba Lytvyn works for MuSE/MAGIC. The authors declare no other interests.

\section{Funding}

This project did not receive dedicated funding.

\section{References}

1. Garritty C, Gartlehner G, Kamel C, King VJ, Nussbaumer-Streit B, Stevens A, et al. Cochrane Rapid Reviews: interim guidance from the Cochrane Rapid Reviews Methods Group. March 2020. Available from methods.cochrane.org/rapidreviews/cochrane-rr-methods (accessed 18 August 2020).

2. The statement of principles for consumer involvement in Cochrane. July 2017. consumers.cochrane.org/news/statement-principlesconsumer-involvement-cochrane.

3. Cochrane Consumer Network. consumers.cochrane.org (accessed 18 August 2020).

4. Pollock A, Campbell P, Struthers C, Synnot A, Nunn J, Hill S, et al. Development of the ACTIVE framework to describe stakeholder involvement in systematic reviews. Journal of Health Services Research and Policy 2019;24(4):245-55. https://doi. org/10.1177/1355819619841647

5. Cochrane. Cochrane Training: consumer involvement. training. cochrane.org/online-learning/consumer-involvement (accessed 18 August 2020). 


\section{COVID-NMA: a collaborative COVID-19 living evidence project}

Authors: Isabelle Boutron ${ }^{1,2}$, David Tovey ${ }^{2}$, Laura De Nale ${ }^{2}$, Anna Chaimani ${ }^{1,2}$, Declan Devane ${ }^{3}$, Joerg J Meerpohl',5, Gabriel Rada ${ }^{6}$, Asbjørn Hróbjartsson 7 , Giacomo Grasselli, ${ }^{8,9}$, Philippe Ravaud ${ }^{1}$, for the COVID-NMA Consortium

Participating groups: Cochrane France, Cochrane Germany, Cochrane Chile, Cochrane Ireland, Cochrane Bias Methods Group, Cochrane South Africa, Cochrane Response, Cochrane Central Executive, Centre for EvidenceBased Medicine Odense, Epistemonikos Foundation, University of Milan, French National Centre for Scientific Research (CNRS)

\section{Corresponding Author: Isabelle Boutron: isabelle.boutron@aphp.fr}

\section{Introduction and background}

COVID-19 has been challenging healthcare and welfare systems all over the world. A huge amount of new research has been initiated, with heterogeneous and frequently small, short trials, limited coordination and a rapidly evolving evidence base. In this context, healthcare professionals, policymakers, politicians, and guidelines developers have to make decisions that have a direct impact on the health of millions of individuals. The COVID-NMA project aims to help them face this emerging situation by producing up-to-date, trustworthy evidence.

The project provides a living mapping of ongoing randomized controlled trials (RCTs) as well as a comprehensive, critical, up-to-date synthesis of all available evidence about the efficacy and safety of interventions for the prevention and treatment of COVID-19. All our data are made publicly available on a platform (covid-nma.com), updated weekly.

COVID-NMA is an international initiative working in conjunction with the World Health Organization (WHO) and led by a team of researchers from Cochrane. In particular:

- Cochrane France: Professors Isabelle Boutron and Philippe Ravaud lead the programme and supervise the various activities. With the support of the Editor in Chief of the Cochrane Library, they initiated the important collaboration with the WHO.

- The Directors of Cochrane Germany, Cochrane Chile, and Cochrane Ireland, the ex-Editor in Chief of the Cochrane Library and convenors from the Cochrane Bias Methods Group are members of the Steering Committee.

- A team based at Cochrane Germany and the Institute for Evidence in Medicine at the University of Freiburg are responsible for grading the overall certainty of the evidence for each outcome using the GRADE classification.[1]

- The search for relevant studies is based on the L.OVE Platform (app.iloveevidence.com/loves/5e6fdb9669c00e4ac072701d), which is managed by the Director of Cochrane Chile.

- A team from Cochrane South Africa is involved in the registration and screening tasks.

- A team from Cochrane Response provides its support for data extraction.

- A team from the Cochrane Bias Methods Group is involved in the quality control process for extracted data.

Other research teams from the Centre for Evidence-Based Medicine Odense (Denmark), the Epistemonikos Foundation (Chile), the University of Milan (Italy), and the French National Centre for Scientific Research (CNRS; France) are also essential members of the consortium (covid-nma.com/team).

\section{Key activities and strategies}

Our project relies on three main pillars: a living mapping of all registered trial evidence to help funders and researchers planning future trials; a living systematic review of trial evidence to support healthcare decision making; and a living monitoring/feedback of trial transparency to improve future research.

\section{Living mapping of registered trials}

Every week we search and extract data from the WHO International Clinical Trials Registry Platform (ICTRP; who.int/clinical-trialsregistry-platform) to identify all RCTs evaluating the effectiveness of interventions for preventing and treating COVID-19 (including rehabilitation) and trials assessing vaccines. We record registered

${ }^{1}$ Centre of Research Epidemiology and Statistics, University of Paris, France; ${ }^{2}$ Cochrane France, France; ${ }^{3}$ National University of Ireland Galway, Ireland; ${ }^{4}$ University of Freiburg, Germany; ${ }^{5}$ Cochrane Germany, Cochrane Germany Foundation, Germany; ${ }^{6}$ Pontificia Universidad Católica de Chile, Chile; ${ }^{7}$ University of Southern Denmark and Odense University Hospital, Denmark; ${ }^{8}$ University of Milan, Italy; ${ }^{9}$ Fondazione IRCCS Cà Granda Ospedale Maggiore Policlinico, Italy 
trial characteristics and feed these into two interactive data visualizations developed by a team from the LIRIS laboratory (liris. cnrs.fr) in the context of a large collaboration with the CNRS. These are available online and are updated weekly: the data visualization on treatments allows us to filter trials by the country in which they are taking place, design, disease severity in trial participants and type of pharmacological treatment being studied (covid-nma.com/ dataviz). We also produce living network maps of these studies (covid-nma.com/networks). The data visualization on vaccines enables users to filter trials by country in which they are taking place, registration date, trial design, recruitment status, phase of the vaccine development process, type of vaccine, and type of participants involved in the trial (covid-nma.com/vaccines/ mapping).

This way of presenting data is useful for researchers planning clinical trials, to identify easily where there is a gap in evidence, for research funders deciding where to dedicate resources for future clinical trials, or for researchers planning systematic reviews and meta-analyses to know which trials are ongoing and when to expect results for their analyses.

\section{Living synthesis of trial results}

We defined a very broad research question, that is, the effects of interventions for the prevention and treatment of COVID-19. We initially performed an extensive search on PubMed, CNKI, medRxiv and ChinaXiv, which we used as primary sources. However, following an evaluation of the sensitivity of our secondary sources, which allowed us to identify $100 \%$ of the RCTs previously included, we updated our search strategy: every day we browse the L.OVE platform (app.iloveevidence.com/ loves/5e6fdb9669c00e4ac072701d) and the Cochrane COVID-19 Study Register (covid-19.cochrane.org) to identify results of RCTs. We have already screened more than 45,000 records.

We collect key data and incorporate this into our evidence syntheses and systematically assess the risk of bias of trials using the Risk of Bias 2 tool.[2] On the COVID-NMA website is a list of treatment comparisons, general characteristics of each trial including a risk of bias assessment, forest plots with results from pairwise meta-analyses, GRADE evidence profile, and 'Summary of findings' tables for all the main outcomes of interest to patients and decision makers. Where appropriate we will undertake network meta-analysis to synthesize the available trial results and compare simultaneously all possible interventions that could be used in the same clinical scenario.

We contact all COVID-19 trial authors to request their protocols and to inform them of the outcomes considered in the review. Our outcome selection is based on the core outcome sets identified through the COMET initiative (comet-initiative.org/studies/ details/1538) and on the results of the research mapping that describes the outcomes assessed in ongoing trials. We are aware that new outcomes will arise over time.

We also contact trial authors with results available to request any additional missing information and we use this to update our reviews accordingly. We encourage investigators to share their individual-patient data at no cost, thanks to our collaboration with Vivli (vivli.org).

We set up a strong process to ensure both quality and efficiency. We separated the process into different tasks and set up a team for each task, led by a senior researcher. We developed an internal and external quality control process for data collection and extraction, in collaboration with the Cochrane Bias Methods Group.

\section{Living monitoring of trial transparency}

We are also recording data related to methodological quality and transparency of the trials, and we will send both individual feedback and community-aggregated results to the trial authors. We hope this will help to improve future research.

\section{Outcomes and impact of activities}

The main outcomes and impact of our project can be summarized as follows.

- We are registering our protocol and related sub-protocols (for each research question) as Cochrane Reviews. The master protocol and the sub-protocol for treatments have been submitted for review by the Cochrane Central Editorial Service.

[3] We are currently working on a sub-protocol related to preventive interventions and on two other sub-protocols on treatment with monoclonal antibodies (IL-1/IL-6 inhibitors).

- Mapping of outcomes evaluated in COVID-19 trials has demonstrated the diversity and lack of consistency of assessed outcomes in the initial trials, which is a significant barrier to evidence synthesis. Communication with trial authors will encourage greater transparency and will also enforce the inclusion of agreed outcomes of most importance to patients and decision makers and thereby improve the quality and usefulness of future research.

- Our data visualizations have enabled researchers and funders to recognize evidence gaps and also to avoid unwarranted duplication of effort.

- The evidence syntheses have enabled decision makers to see all the RCTs that address questions related to prevention, treatment, rehabilitation and vaccines, and to explore the data in order to guide decisions. The synthesis has already been used by guidelines and health technology assessment (HTA) bodies to develop their treatment recommendations. As an example, we are working with the WHO as well as the UK National Institute for Health and Care Excellence (NICE). 
- The importance and quality of our work have been recognized by many researchers all over the world. As a result, some of them have offered to help out with some of the tasks on a voluntary basis. Some authors and investigators have congratulated us on the quality and value of our work and agreed to share their data and results to improve our evidence synthesis.

- Feedback has also helped to focus our work better: considering the increase in RCTs with available results, we have recently decided to change our search strategy and to exclude nonrandomized studies from the living systematic review.[4]

\section{Lessons for the future: sustainability and transferability}

During each phase of the project we encountered a number of challenges that had to be rapidly tackled, and we have learned some important lessons.

- In the early phase of the pandemic there was a huge amount of work to be performed while all the people involved in the project were based in different countries and working from home during lockdown. To ensure efficient co-ordination, several processes had to be developed from scratch and we set up a co-ordinating team of senior researchers to supervise all the work remotely. We learned that research questions change as experience and knowledge of the new virus increase. As a result, we had to adapt to this evolution and to modify our protocol, but this also allowed us to improve our search strategy and processes.

- We learned to build capacity to manage the workload: in an early phase we developed our project with the support of several volunteers who dedicated some of their time to screen, extract data, conduct analysis, etc. However, volunteers eventually needed to return to their usual activities. In order to keep the project running, we involved colleagues from other research centres, and we secured some funding to support this. Sustainability remains one of our challenges, especially with the increasing amount of data available.

- The heterogeneity of trials and the diversity and complete lack of consistency of assessed outcomes in the initial trials are significant barriers to evidence synthesis. We learned the importance of agreed outcomes: we are now able to contact trial authors before or just after their trial has begun and raise their awareness of the data and outcomes considered in our review.

- Changing the culture and getting researchers to share their data, even in a global pandemic, takes time: we encountered difficulties in obtaining feedback from investigators of ongoing trials and from authors of completed trials. We are now setting up a secure process to allow investigators to share their data at no cost.

Through this project we are building a new evidence ecosystem that will be useful to tackle future pandemics.[5]

\section{Additional resources}

- COVID-NMA: covid-nma.com

- COVID-NMA on Zenodo: zenodo.org/communities/covid-nma

- COVID-NMA on Twitter: twitter.com/covidnma

\section{Acknowledgements}

Solaf Alawadhi (Center of Research in Epidemiology and Statistics, University of Paris, France); Sihem Amer-Yahia (Grenoble Informatics Laboratory, France); Chiara Arienti (Center for Health Regulatory Policies, IRCSS, Italy); David Auber (LaBRI, University of Bordeaux I, France); Camila Ávila (Epistemonikos Foundation, Chile); Aïda Bafeta (Centre of Research in Epidemiology and Statistics, France); Fulvia Baldassarre (McMaster University, France); Rita Banzi (Istituto di Ricerche Farmacologiche Mario Negri IRCCS, Italy); Julien Barnier (Max Weber Center, France); Julia Baudry (Center of Research in Epidemiology and Statistics, France); Hanna Bergman (Cochrane Response); Claudia Bollig (Cochrane Germany, Cochrane Germany Foundation, Germany); Hillary Bonnet (University of Paris and Bordeaux PharmacoEpi, France); Marinette Bouet (LIMOS, Clermont Auvergne University, CNRS, France); Mohand Boughanem (Institut de Recherche en Informatique de Toulouse, France); Isabelle Boutron (Cochrane France and Center of Research in Epidemiology and Statistics, University of Paris, France); Brian Buckley (Cochrane Response); Guillaume Cabanac (Institut de Recherche en Informatique de Toulouse, France); Anna Chaimani (Cochrane France and University of Paris, France); Sarah Charpy (Centre of Research in Epidemiology and Statistics, France); David Chavalarias (ISC-PIF, CNRS, France); Yaolong Chen (WHO Collaborating Centre for Guideline Implementation and Knowledge Translation and Chinese GRADE Centre, Lanzhou University, China); Astrid Chevance (Centre of Research in Epidemiology and Statistics, France); Sarah Cohen-Boulakia (LRI, CNRS, Université Paris-Saclay, France); Elise Cogo (Cochrane Response); Françoise Conil (LIRIS, CNRIS, Université Claude Bernard Lyon 1, France); Emmanuel Coquery (LIRIS, CNRIS, Université Claude Bernard Lyon 1, France); Mauricia Davidson (Cochrane France and Center of Research in Epidemiology and Statistics, France); Laura De Nale (Cochrane France); Declan Devane (Evidence Synthesis Ireland, Cochrane Ireland, and National University of Ireland, Ireland); Elise Diard (Cochrane Response); Taoufiq Dkaki (IRIT, Université Toulouse III Paul Sabatier, France); Bastien Doreau (LIMOS, CNRS, Université Clermont Auvergne, France); Merwan El Asri (LRI, CNRS, Université Paris-Saclay, France); Theodoros Evrenoglou (Cochrane France and University of Paris, France); Alice Fabbri (Centre for Evidence Based Medicine Odense, University of Southern Denmark and Odense University Hospital, Denmark); Gilles Feron (French National Research Institute for Agriculture, Food and Environment, France); Gabriel Ferrand (Cochrane France); Leopold Fezeu (Centre of Research in Epidemiology and Statistics, France); Mathilde Fouet (Hôpital d'Instruction des Armées Percy, France); Joly Ghanawi (CAMARADES, University of Edinburgh, UK); Lina Ghosn (Cochrane France); Robin Featherstone (Cochrane Central Executive); Carolina Graña (Cochrane France, and Centre of Research in Epidemiology and Statistics, France); Giacomo Grasselli (Fondazione IRCCS Ca' Granda Ospedale Maggiore Policlinico and University of Milan, Italy); François Grolleau (University of Paris, France); Benoit Groz (LRI, CNRS, Université Paris-Saclay, France); Mohand-Saïd Hacid (LIRIS, CNRS, Université Claude Bernard 
Lyon 1, France); Candyce Hamel (Cochrane Response); Camilla Hansen (Centre for Evidence Based Medicine Odense, University of Southern Denmark and Odense University Hospital, Denmark); Nicholas Henschke (Cochrane Response); Ameer Hohlfeld (Cochrane South Africa, South African Medical Research Council, South Africa); Asbjørn Hróbjartsson (Centre for Evidence Based Medicine Odense, University of Southern Denmark and Odense University Hospital, Denmark); Chantal Julia (Centre of Research in Epidemiology and Statistics, France); Dimitris Mavridis (University of Ioannina, Greece); Joerg J Meerpohl (Cochrane Germany, Cochrane Germany Foundation, and University of Freiburg, Germany); Brice Meyer (Bordeaux PharmacoEpi, France); Silvia Minozzi (University of Milan, Italy); Jose G Moreno (Institut de Recherche en Informatique de Toulouse, France); Nivantha Naidoo (Centre of Research in Epidemiology and Statistics, France); Van Thu Nguyen (Cochrane France); Theodora Oikonomidi (Cochrane France and University of Paris, France); Matthew Page (Monash University, Australia); Jennifer Petkovic (Cochrane Response); Elizabeth Pienaar (University of Milan, Italy); Olivier Pierre (Centre of Research in Epidemiology and Statistics, France); Katrin Probyn (Cochrane Response); Fiona Quirke (Health Research Board-Trials Methodology Research Network, Ireland); Gabriel Rada (Epistemonikos Foundation and Cochrane Chile/Pontificia Universidad Católica de Chile, Chile); Philippe Ravaud (Cochrane France and University of Paris and Centre of Research in Epidemiology and Statistics, France); Pierre Ripoll (LIRIS, CNRS, France); Carolina Riveros (Cochrane France and Centre of Research in Epidemiology and Statistics, France); Philippe Rivière (LRIS, CNRS, France); Marie Sauvant (LIMOS, CNRS, France); Jelena Savovic (University of Bristol/NIHR CLAHRC West/University Hospitals Bristol and Weston NHS Foundation Trust, UK); Christine Schmucker (University of Freiburg, Germany); Yanina Sguassero (Cochrane Response); Jonathan Sterne (University of Bristol, UK); Farouk Toumani (LIMOS, CNRS, France); David Tovey (Cochrane France); Gemma Villanueva (Cochrane Response); Romain Vuillemot (LIRIS, CNRS, France); Jun Xia (University of Nottingham Ninhbo, China); Xuan Yu (WHO Collaborating Centre for Guideline Implementation and Knowledge Translation/Chinese GRADE Centre, Lanzhou University, China); Emina Zoletic (University of Paris/Center of Research in Epidemiology and Statistics, France); Pierre Zweigenbaum (LIMSI, CNRS, France).

\section{Declarations of interest}

IB is Director of Cochrane France, a member of the Cochrane Editorial Board, and Convener of the Cochrane Bias Methods Group; DT was previously Editor in Chief of Cochrane (2009-2019); AC is Convener of the Cochrane Comparing Multiple Interventions Methods Group and Cochrane Statistics Methods Group; DD is Director of Cochrane Ireland; JM is Director of Cochrane Germany; GR is Director of Cochrane Chile; $\mathrm{AH}$ is Convener of the Cochrane Bias Methods Group. The authors declare that they have no other competing interests.

\section{Funding}

This work received some funding from the Agence Nationale de la Recherche (ANR), the World Health Organization (WHO), Cochrane France, Center of Research in Epidemiology and Statistics (CRESS), Centre d'Epidémiologie Clinique (GHU Cochin, Hôtel Dieu, Assistance Publique Hôpitaux de Paris (APHP) and Université de Paris), and the Federal Ministry of Health, Germany.

\section{References}

1. GRADEpro Guideline Development Tool [Software]. McMaster University, 2020 (developed by Evidence Prime Inc). Available from gradepro.org.

2. Sterne JA, Savović J, Page MJ, Elbers RG, Blencowe NS, Boutron I, et al. RoB 2: a revised tool for assessing risk of bias in randomised trials. BMJ 2019; 366:l4898. https://doi.org/10.1136/bmj.14898

3. Wakeford H, Dooley C, Stephani A-M, Marshall R, Featherstone R, Walshe D, et al. The Central Editorial Service: a collaborative editorial process for publishing high-priority Cochrane reviews. In: Collaborating in response to COVID-19: editorial and methods initiatives across Cochrane. Cochrane Database of Systematic Reviews 2020;(12 Suppl 1). https://doi.org/10.1002/14651858.CD202002

4. Boutron I, Chaimani A, Meerpohl JJ, Hróbjartsson A, Devane D, Rada G, et al. Interventions for preventing and treating COVID-19: protocol for a living mapping of research and a living systematic review. Zenodo 2020;September 8. https://doi.org/10.5281/ zenodo.3744599

5. Boutron I, Chaimani A, Meerpohl JJ, Hróbjartsson A, Devane D, Rada $G$, et al. The COVID-NMA project: building an evidence ecosystem for the COVID-19 pandemic. Annals of Internal Medicine 2020;September 15. https://doi.org/10.7326/M20-5261 


\title{
Cochrane COVID-19 living systematic reviews: why, how, and what has been done?
}

\author{
Authors: Tari Turner ${ }^{1}$, Steve McDonald ${ }^{1}$ \\ Participating groups: Cochrane Australia, Living Evidence Network \\ Corresponding Author: Tari Turner: tari.turner@monash.edu
}

\section{Introduction and background}

By early April 2020, three months after the SARS-CoV-2 outbreak was declared a 'Public Health Emergency of International Concern', more than a million cases of COVID-19 and nearly 70,000 deaths had been recorded worldwide.[1] In the same period, over 300 COVID-19 clinical trials were registered on ClinicalTrials.gov, a number that had exceeded 3000 by September 2020 .

In the early phase of the pandemic there was very little direct evidence to guide treatment decisions. However, a rapidly increasing volume of COVID-19 research is appearing every day,[2] presenting a substantial challenge to authors of systematic reviews. Since the credibility and utility of systematic reviews to guide decision-making relies on their currency, the rapid accumulation and dissemination of evidence relating to COVID-19 has emphasized the importance of adopting living approaches.

'Living' evidence synthesis methods were developed to enable systematic reviews to be continually updated.[3] Living approaches are ideal for the COVID-19 crisis because the area is a priority for decision-making, review conclusions are likely to change as new evidence emerges, and new evidence is available on a daily basis.[4]

Living systematic reviews (LSRs) are now being undertaken in many areas of health.[4] Cochrane has led the way in conducting LSRs, as well as in developing and evaluating their methods. Here, we provide a brief overview of methods for Cochrane LSRs and highlight some examples of living reviews conducted by Cochrane that are informing the response to COVID-19.

\section{Key activities and strategies}

A Cochrane LSR is a Cochrane review that is continually updated, incorporating relevant new evidence as it becomes available. Cochrane LSRs:

- are underpinned by continual, active monitoring of the evidence;

- immediately include any new important evidence as it is identified; and
- are supported by up-to-date communication about the status of the review and any new evidence being incorporated.

Core methods for Cochrane LSRs are essentially the same as other Cochrane reviews, however, LSRs also include explicit, transparent and predefined decisions on how frequently new evidence is sought and screened, and when and how new evidence is incorporated into the review. Some Cochrane reviews also make use of Cochrane Crowd and machine learning techniques to improve the efficiency of review processes (community.cochrane.org/help/tools-and-software).

Living systematic reviews were first piloted in Cochrane in 2017, and an evaluation of the pilots was published in 2019.[5] There are now nine LSRs and living network meta-analyses in the Cochrane Library, with many more in development.

Guidance on methods for production and publication of Cochrane LSRs, and a range of other resources, are available at community. cochrane.org/review-production/production-resources/livingsystematic-reviews for author and editorial teams interested in conducting Cochrane LSRs.

\section{Outcomes and impact of activities}

Recognizing the value of living evidence synthesis approaches, several Cochrane groups have produced and are updating LSRS to inform decision-making in COVID-19. These include reviews addressing key issues such as:

- convalescent plasma or hyperimmune immunoglobulin for people with COVID-19;[6]

- signs and symptoms to determine if a patient presenting in primary care or hospital outpatient settings has COVID-19 disease;[7]

- antibody tests for identification of current and past infection with SARS-CoV-2.[8]

Cochrane Rehabilitation (rehabilitation.cochrane.org) is also conducting a series of rapid LSRs to provide up-to-date evidence on rehabilitation and COVID-19.[9]

${ }^{1}$ Cochrane Australia, Monash University, Australia 
The Cochrane LSR of convalescent plasma highlights the rapid evolution of the evidence base.[6] The first version, published in mid-May, included eight studies, nearly all case series, involving just 32 participants. Two months later, the first update included 20 studies (one randomized trial and 19 non-randomized studies) comprising over 5000 participants and identified a further 50 ongoing randomized trials.

Groups outside Cochrane have also recognized the value of living approaches to evidence syntheses during the COVID-19 pandemic, and many teams are developing LSRs to support health decisions. These groups include The LIVING Project,[10] COVID-NMA (covid-nma.com),[11] and the COVID-19 L.OVE Working Group (epistemonikos.cl/living-evidence).

Living evidence synthesis methods are also being applied to guideline development for COVID-19. For example, Cochrane Australia is part of the Australian National COVID-19 Clinical Evidence Taskforce, developing living evidence-based guidelines for treatment of people with COVID-19 (covid19evidence.net.au). These guidelines have been updated weekly since early April and now comprise over 90 recommendations, informed by randomized trials and systematic reviews.

\section{Lessons for the future: sustainability and transferability}

While there is no doubt that rigorous living systematic reviews are crucial to ensuring evidence-based responses to COVID-19, many questions remain. Ongoing evidence surveillance and updating is at the core of the value of LSRs - as yet we don't know how sustainable efforts to conduct and maintain COVID-19-relevant LSRs will be. In many cases, the period over which LSRs can be maintained will be linked to ongoing funding, and perhaps also, to the enthusiasm and commitment of authors.

A particular challenge in the context of COVID-19 LSRs is how to manage the rapidly evolving nature of the evidence base. At inception, for many of the Cochrane COVID-19 LSRs, evidence was derived predominantly from case series and modelling studies. For reviews of interventions, these have been followed by comparative observational studies and, more recently, by randomized trials. Clear methods and processes are needed for how LSRs will manage changes in the type of eligible evidence over the lifecycle of the review.

Evidence surveillance has its own unique challenges, not least establishing efficient and reliable methods for deduplicating records and linking multiple reports (registry entries, protocols, preprints, journal articles) to the same study. Managing and documenting these processes so as to maintain an accurate PRISMA flow diagram is time-consuming and complicated.
Further clarity is needed around editorial processes and peerreview policies, particularly finding the right balance between quality assurance and timeliness, depending on the extent of changes from one version to the next. How to maintain internal consistency and avoid errors despite frequent, piecemeal changes, and how to ensure that the overall integrity of the review is preserved, are important considerations in deciding how rapidly to update and publish new versions.

Finally, there are questions about when to transition LSRs from living mode to more traditional, less frequent, models of updating. Following the publication of large, high-quality studies, it is likely that the evidence base will stabilize with respect to the review's overall conclusions-the accumulation of numerous, smaller studies will add to the evidence base without necessarily impacting on the conclusions. Establishing criteria for updating this category of reviews will need to balance the desire for reviews to be current with respect to all available evidence with the additional workload involved in maintaining reviews in which the conclusions may change little. This has implications for how author teams communicate the status of the review to readers, as well as establishing criteria for determining when the evidence base is stable and when questions are no longer considered high priority.

The application of living evidence synthesis models during the pandemic provides a very important opportunity to develop methods for LSRs further, and to learn more about the situations in which LSRs are most useful and most feasible.

\section{Additional resources}

Living systematic reviews resources community.cochrane.org/ review-production/production-resources/living-systematic-reviews

\section{Acknowledgements}

With thanks to our colleagues Annie Synnot and Julian Elliott for their work on developing the methods for living systematic reviews, and to all the LSR author teams for their ongoing efforts to conduct and maintain Cochrane LSRs.

\section{Declarations of interest}

TT is a Senior Research Fellow and SM is Co-Director of Cochrane Australia, based at Monash University in Melbourne, Australia, and funded by the Australian National Health and Medical Research Council. Both are contributing to the Australian National COVID-19 Clinical Evidence Taskforce which is funded by: Australian Government Department of Health; Victorian Government Department of Health and Human Services; The lan Potter Foundation; Walter Cottman Endowment Fund, managed by Equity Trustees; Lord Mayors' Charitable Foundation.

\section{Funding}

Cochrane Australia is funded by the Australian National Health and Medical Research Council. 


\section{References}

1. Salyer K. COVID-19: what to know about the coronavirus pandemic on 6 April. World Economic Forum. 6 April 2020. www.weforum.org/ agenda/2020/04/covid-19-what-to-know-about-the-coronaviruspandemic-on-6-april

2. Fidahic M, Nujic D, Runjic R, Civljak M, Markotic F, Lovric Makaric Z, et al. Research methodology and characteristics of journal articles with original data, preprint articles and registered clinical trial protocols about COVID-19. BMC Medical Research Methodology 2020;20(1):161. https://doi.org/10.1186/s12874-020-01047-2

3. Elliott JH, Synnot A, Turner T, Simmonds M, Akl EA, McDonald S, et al. Living systematic review: 1. Introduction-the why, what, when, and how. Journal of Clinical Epidemiology 2017;91:23-30. https://doi. org/10.1016/j.jclinepi.2017.08.010

4. Khamis AM, Kahale LA, Pardo-Hernandez H, Schunemann HJ, Akl EA. Methods of conduct and reporting of living systematic reviews: a protocol for a living methodological survey. F1000 Research 2019;8:221. https://doi.org/10.12688/f1000research.18005.2

5. Millard T, Synnot A, Elliott J, Green S, McDonald S, Turner T. Feasibility and acceptability of living systematic reviews: results from a mixedmethods evaluation. Systematic Reviews 2019;8:325. https://doi. org/10.1186/s13643-019-1248-5

6. Piechotta V, Chai KL, Valk SJ, Doree C, Monsef I, Wood EM, et al. Convalescent plasma or hyperimmune immunoglobulin for people with COVID-19: a living systematic review. Cochrane Database of Systematic Reviews 2020;(7):CD013600. https://doi.org/10.1002/14651858.

CD013600.pub2
7. Struyf T, Deeks JJ, Dinnes J, Takwoingi Y, Davenport C, Leeflang MM, et al. Signs and symptoms to determine if a patient presenting in primary care or hospital outpatient settings has COVID-19 disease. Cochrane Database of Systematic Reviews 2020;(7):CD013665. https:// doi.org/10.1002/14651858.CD013665

8. Deeks JJ, Dinnes J, Takwoingi Y, Davenport C, Spijker R, TaylorPhillips S, et al. Antibody tests for identification of current and past infection with SARS-CoV-2. Cochrane Database of Systematic Reviews 2020;(6):CD013652 https://doi.org/10.1002/14651858.CD013652

\section{Negrini S, Arienti C, Cusick A, Kiekens C, International} Multiprofessional Steering Committee of Cochrane Rehabilitation REHCOVER action. REH-COVER (Rehabilitation - COVID-19 Evidence-based Response) action to recover functioning during/after COVID-19 and its treatments. In: Collaborating in response to COVID-19: editorial and methods initiatives across Cochrane. Cochrane Database of Systematic Reviews 2020;(12 Suppl 1). https://doi.org/10.1002/14651858.CD202002

10. Juul S, Nielson EE, Feinberg J, Siddiqui F, Jørgensen CK, Barot E, et al. Interventions for treatment of COVID-19: a living systematic review with meta-analyses and trial sequential analyses (The LIVING Project). PLOS Medicine 2020;17(9):e1003293. https://doi.org/10.1371/journal. pmed.1003293

11. Boutron I, Tovey D, De Nale L, Chaimani A, Devane D, Meerpohl JJ, et al. COVID-NMA: a collaborative COVID-19 living evidence project. In: Collaborating in response to COVID-19: editorial and methods initiatives across Cochrane. Cochrane Database of Systematic Reviews 2020;(12 Suppl 1). https://doi.org/10.1002/14651858.CD202002 


\title{
Rapid review methods guidance aids in Cochrane's quick response to the COVID-19 crisis
}

\begin{abstract}
Authors: Chantelle Garritty ${ }^{1,2}$, Gerald Gartlehner ${ }^{3,4}$, Barbara Nussbaumer-Streit ${ }^{3}$, Valerie J King ${ }^{5}$, Chris Kamel ${ }^{6}$, Adrienne Stevens ${ }^{7}$, Declan Devane ${ }^{8}$, Candyce Hamel ${ }^{1,2}$, Lisa Affengruber ${ }^{3}$
\end{abstract}

Participating groups: Cochrane Rapid Reviews Methods Group

Corresponding Author: Chantelle Garritty: cgarritty@ohri.ca

\section{Introduction and background}

Rapid reviews have emerged as an efficient tool to get evidence to decision-makers more quickly and are part of the knowledge synthesis family.[1] Rapid Reviews have been described as a type of knowledge synthesis in which systematic review methods are streamlined, and processes accelerated to complete the review more quickly.[2-5] Policymakers are increasingly using rapid reviews in their daily decision-making,[6-9] with national and international health agencies using rapid reviews to inform guideline recommendations.[10-12]

Since 2015, the Cochrane Rapid Reviews Methods Group (RRMG) has served as a discussion forum and has led the development of rapid review methods.[13-15] In 2018, Cochrane's Strategy to 2020 (community.cochrane.org/organizational-info/resources/ strategy-2020) identified the need to explore and, potentially, implement guidance and systems for officially producing Cochrane rapid reviews. The strategy outlined the need to develop recommendations regarding which methods can be abbreviated to expedite publication. During 2019, the RRMG conducted a suite of related methodological work, including two scoping reviews,[16,17] and two primary methods studies.[18,19] Designed to fill methodological gaps and provide guidance on conducting rapid reviews, collectively this research formed the evidentiary base for a subsequent rapid review methods options survey sent to 119 representatives from 20 Cochrane entities in the fall of 2019. Respondents were asked to rate and rank rapid review methods across the stages of conduct. Based on survey results from 63 respondents (53\% response rate), we proposed interim guidance comprised of 26 specific recommendations to support the conduct of rapid reviews. Further, we proposed that a Cochrane rapid review be defined as, "a form of knowledge synthesis that accelerates the process of conducting a traditional systematic review through streamlining or omitting specific methods to produce evidence for stakeholders in a resource-efficient manner".[17] This guidance emphasizes the involvement of key stakeholders throughout the rapid review process and promotes a flexible, iterative approach that can be tailored for various urgent and emergent health decision-making scenarios.

\section{Key activities and strategies}

We undertook the following activities during the COVID-19 pandemic.

1. In early March 2020, the RRMG completed work on the Cochrane rapid review methods interim gußidance,[20] which coincided with the global pandemic's unfolding. This was the catalyst to Cochrane encouraging the early release of the guidance on 23 March 2020.

2. As part of their overall response to COVID-19, Cochrane developed internal and external processes to accommodate the production of rapid reviews, among other products. It meant that the interim guidance was made available as part of resources for author teams on the COVID Rapid Reviews website (covidreviews.cochrane.org). More specifically, the guidance was integrated into the protocol template for Cochrane rapid reviews.

3. RRMG convenors have been actively involved in leading the development of Cochrane COVID-19 rapid reviews since the outset of the pandemic.[21-24]

4. RRMG convenors have provided methodological support to various author teams undertaking COVID-19 rapid reviews produced within Cochrane and external teams.

${ }^{1}$ Ottawa Hospital Research Institute, Canada; ${ }^{2}$ University of Split School of Medicine, Croatia; ${ }^{3}$ Cochrane Austria, Danube University Krems, Austria; ${ }^{4}$ RTI International, USA; ${ }^{5}$ Oregon Health \& Science University, USA; ${ }^{6} \mathrm{CADTH}$, Canada; ${ }^{7}$ Cochrane Canada, McMaster University, Canada; ${ }^{8} \mathrm{Cochrane}$ Ireland, Evidence Synthesis Ireland, National University of Ireland (NUI Galway), Ireland 
5. RRMG convenors have directly supported Cochrane COVID-19 initiatives including the initial Cochrane COVID-19 Response Working Group formed in the early days of the pandemic to help guide Cochrane's response. Further, one of the RRMG convenors is a member of the steering committee of the 'COVID NMA Living mapping and living systematic review of Covid-19 studies' initiative.[25]

6. Over the past six months, RRMG convenors have delivered several information and training sessions via webinars related to the interim Cochrane rapid review methods guidance or specific Cochrane COVID-19 rapid reviews, with all events well-attended.

\section{Outcomes and impact of activities}

Development of the interim Cochrane rapid review methods guidance, made publicly available, has been an impactful outcome of our work and has been beneficial to Cochrane's response to COVID-19. This guidance has been formally cited more than 25 times in the past six months, and the Cochrane RRMG website page that houses this guidance has been viewed nearly 2300 times since it was posted. To our knowledge, this rapid review methods guidance is the first that provides clear, actionable recommendations, based on empirical evidence, evaluating RR methods to date and with expert input. Importantly, this guidance is being actively used to develop Cochrane rapid reviews to address pressing questions posed by international stakeholders. Moreover, these rapid reviews have attained extremely high Altmetric Attention Scores, indicating that they have received substantial online attention. Contributing to this was Cochrane's decision to make these rapid reviews freely accessible from the outset. Although COVID-19 may have been the impetus to releasing this guidance, the proposed recommendations are relevant for any circumstance where decision-making needs to be made in weeks to a few months. COVID-19 and the use of this guidance has underscored the need for flexible guidance that can be tailored as appropriate, yet still meets minimum standards. While this guidance was developed for Cochrane, we suggest that it is relevant and of interest for a wide audience of rapid review authors, many of whom look to Cochrane for methods expertise.

\section{Lessons for the future: sustainability and transferability}

We recognize that further refinements are needed regarding this interim guidance. In terms of next steps, we aim to solicit feedback on the guidance's perceived utility as applied in urgent, real-time rapid review scenarios. It will also be important that we adapt the guidance beyond interventions of effectiveness to other review types, such as rapid reviews of diagnostic test accuracy or screening.[26] In doing so, specific rapid review types will require unique considerations.[27] Beyond this, there are other challenges to the conduct of rapid reviews that further merit discussion.[28] Because best practice is limited by the lack of currently available evidence for some methods shortcuts taken in rapid reviews, this guidance will need to be updated as additional abbreviated methods are evaluated. There is a need to highlight uncertainties in rapid review methods so future research questions can be identified and prioritized. A rapid review methodology priority setting partnership (Priority III), led by Evidence Synthesis Ireland/ Cochrane Ireland, has set out to do this with two RRMG convenors serving on the Steering Group.[29] COVID-19 is a clear and current example where decisions need to be made faster than traditional systematic reviews can support. Endorsing a rapid review approach alongside interim methods guidance has demonstrated Cochrane's ability to respond quickly as a world leader in knowledge synthesis, and well positions Cochrane to respond to future urgent or emergent health crises.

\section{Additional resources}

Cochrane Rapid Review Methods Group: methods.cochrane.org/ rapidreviews

\section{Cochrane COVID Reviews: covidreviews.cochrane.org}

\section{Declarations of interest}

CG, GG, VJK, CK, BN-S, AS, DD are Convenors and CH, LA are Associate Convenors of the Cochrane Rapid Reviews Methods Group. BN-S, DD and GG have authored Cochrane COVID-19 Rapid Reviews. All authors declare no other conflicts of interest.

\section{Funding}

The Rapid Reviews Methods Group was funded through the Cochrane Content Strategy fund to develop the Cochrane Rapid Review methods interim guidance. One project was also funded in part from a Canadian Institutes of Health Research (ClHR) grant (funding research number 142310). There was no other dedicated funding for additional parts of this project.

\section{References}

1. Moher D, Stewart L, Shekelle P. All in the family: systematic reviews, rapid reviews, scoping reviews, realist reviews, and more. Systematic Reviews 2015;4:183. https://doi.org/10.1186/s13643-0150163-7

2. Ganann R, Ciliska D, Thomas H. Expediting systematic reviews: methods and implications of rapid reviews. Implementation Science 2010;5:56. https://doi.org/10.1186/1748-5908-5-56

3. Khangura S, Konnyu K, Cushman R, Grimshaw J, Moher D. Evidence summaries: the evolution of a rapid review approach. Systematic Reviews 2012;1:10. https://doi.org/10.1186/2046-4053-1-10

4. Tricco AC, Antony J, Zarin W, Strifler L, Ghassemi M, Ivory J, et al. A scoping review of rapid review methods. BMC Medicine 2015;13:224. https://doi.org/10.1186/s12916-015-0465-6

5. Tricco AC, Zarin W, Ghassemi M, Nincic V, Lillie E, Page MJ, et al. Same family, different species: methodological conduct and quality varies according to purpose for five types of knowledge synthesis. Journal 
of Clinical Epidemiology 2018;96:133-42. https://doi.org/10.1016/j. jclinepi.2017.10.014

6. Moore G, Redman S, Rudge S, Haynes A. Do policy-makers find commissioned rapid reviews useful? Health Research and Policy Systems 2018;16:17. https://doi.org/10.1186/s12961-018-0293-1

7. Mijumbi-Deve R, Rosenbaum SE, Oxman AD, Lavis JN, Sewankambo NK. Policymaker experiences with rapid response briefs to address health-system and technology questions in Uganda. Health Research Policy and Systems 2017;15:37. https://doi.org/10.1186/s12961-0170200-1

8. Hartling L, Guise J-M, Hempel S, Featherstone R, Mitchell MD, Motu'apuaka ML, et al. Fit for purpose: perspectives on rapid reviews from end-user interviews. Systematic Reviews 2017;6:32. https://doi. org/10.1186/s13643-017-0425-7

9. Peterson K, Floyd N, Ferguson L, Christensen V, Helfand M. User survey finds rapid evidence reviews increased uptake of evidence by Veterans Health Administration leadership to inform fast-paced healthsystem decision-making. Systematic Reviews 2016;5:132. https://doi. org/10.1186/s13643-016-0306-5

10. Thigpen S, Puddy RW, Singer HH, Hall DM. Moving knowledge into action: developing the rapid synthesis and translation process within the interactive systems framework. American Journal of Community Psychology 2012;50:285-94. https://doi.org/10.1007/s10464-012-9537-3

11. Patnode CD, Eder ML, Walsh ES, Viswanathan M, Lin JS. The use of rapid review methods for the U.S. Preventive Services Task Force. American Journal of Preventive Medicine 2018;54:S19-25. https://doi. org/10.1016/j.amepre.2017.07.024

12. Garritty CM, Norris SL, Moher D. Developing WHO rapid advice guidelines in the setting of a public health emergency. Journal of Clinical Epidemiology 2017;82:47-60. https://doi.org/10.1016/j. jclinepi.2016.08.010

13. Garritty C, Stevens A, Gartlehner G, King V, Kamel C, on behalf of the Cochrane Rapid Reviews Methods Group. Cochrane Rapid Reviews Methods Group to play a leading role in guiding the production of informed high-quality, timely research evidence syntheses. Systematic Reviews 2016;5:184. https://doi.org/10.1186/s13643-016-0360-z

14. King J, Garritty C, Stevens A, Nussbaumer-Steit B, Hartling L, Harrod $\mathrm{CS}$, et al. Chapter 2: Performing rapid reviews. In: Tricco AC, Langlois EV, Straus SE, editors. Rapid Reviews to Strengthen Health Policy and Systems: a Practical Guide 2017. Available at www.who.int/alliance-hpsr/ resources/publications/rapid-review-guide

15. Stevens A, Garritty C, Hersi M, Moher D. Developing PRISMA-RR, a reporting guideline for rapid reviews of primary studies (protocol). February 2018. equator-network.org/wp-content/uploads/2018/02/ PRISMA-RR-protocol.pdf

16. Hamel C, Michaud A, Thuku M, Affengruber L, Skidmore B, Nussbaumer-Streit $B$, et al. Few evaluative studies exist examining rapid review methodology across stages of conduct: a systematic scoping review. Journal of Clinical Epidemiology 2020;126:131-40. https://doi. org/10.1016/j.jclinepi.2020.06.027

17. Hamel C, Michaud A, Thaku M, Skidmore B, Stevens A, NussbaumerStreit $B$, et al. Defining rapid reviews: a systematic scoping review and thematic analysis of definitions and defining characteristics of rapid reviews. Journal of Clinical Epidemiology 2020;129:74-85. https://doi. org/10.1016/j.jclinepi.2020.09.041

18. Nussbaumer-Streit B, Klerings I, Dobrescu Al, Persad E, Stevens A, Garritty $C$, et al. Excluding non-English publications from evidencesyntheses did not change conclusions: a meta-epidemiological study. Journal of Clinical Epidemiology 2020;118:42-54. https://doi. org/10.1016/j.jclinepi.2019.10.011

19. Gartlehner G, Affengruber L, Titscher V, Noel-Storr A, Dooley G, Ballarini N, et al. Single-reviewer abstract screening missed 13 percent of relevant studies: a crowd-based, randomized controlled trial. Journal of Clinical Epidemiology 2020;121:20-8. https://doi.org/10.1016/j. jclinepi.2020.01.005

20. Garritty C, Gartlehner G, Kamel C, King VJ, Nussbaumer-Streit B, Stevens A, et al. Cochrane Rapid Reviews Methods Group offers evidence-informed guidance to conduct rapid reviews. Journal of Clinical Epidemiology 2020;130:13-22. https://doi.org/10.1016/j. jclinepi.2020.10.007

21. Nussbaumer-Streit B, Mayr V, Dobrescu Al, Chapman A, Persad $\mathrm{E}$, Klerings I, et al. Quarantine alone or in combination with other public health measures to control COVID-19: a rapid review. Cochrane Database of Systematic Reviews 2020;(9):CD013574. https://doi. org/10.1002/14651858.CD013574.pub2

22. Noone C, McSharry J, Smalle M, Burns A, Dwan K, Devane D, et al. Video calls for reducing social isolation and loneliness in older people: a rapid review. Cochrane Database of Systematic Reviews 2020;(5):CD013632. https://doi.org/10.1002/14651858.CD013632

23. Houghton C, Meskell P, Delaney H, Smalle M, Glenton C, Booth $A$, et al. Barriers and facilitators to healthcare workers' adherence with infection prevention and control (IPC) guidelines for respiratory infectious diseases: a rapid qualitative evidence synthesis. Cochrane Database of Systematic Reviews 2020;(4):CD013582. https://doi. org/10.1002/14651858.CD013582

24. Viswanathan M, Kahwati L, Jahn B, Giger K, Dobrescu Al, Hill C, et al. Universal screening for SARS-CoV-2 infection: a rapid review. Cochrane Database of Systematic Reviews 2020;(9):CD013718. https://doi. org/10.1002/14651858.CD013718

25. Boutron I, Tovey T, De Nale L, Chaimani A, Devane D, Meerpohl JJ, et al. COVID-NMA: a collaborative COVID-19 living evidence project. In: Collaborating in response to COVID-19: editorial and methods initiatives across Cochrane. Cochrane Database of Systematic Reviews 2020;(12 Suppl 1). https://doi.org/10.1002/14651858.CD202002

26. Arevalo-Rodriguez I, Tricco AC, Nussbaumer-Streit B, Steingart KR, Kaunelis D, Alonso-Coello $P$, et al. Developing rapid reviews of diagnostic tests in the time of COVID-19: current knowledge and future steps. In: Collaborating in response to COVID-19: editorial and methods initiatives across Cochrane. Cochrane Database of Systematic Reviews 2020;(12 Suppl 1). https://doi.org/10.1002/14651858.CD202002

27. Arevalo-Rodriguez I, Tricco AC, Steingart KR, Nussbaumer-Streit $B$, Kaunelis D, Alonso-Coello $P$, et al. Challenges of rapid reviews for diagnostic test accuracy questions: a protocol for an international survey and expert consultation. Diagnostic and Prognostic Research 2019;3:7. https://doi.org/10.1186/s41512-019-0052-y 
28. Tricco AC, Garritty CM, Boulos L, Lockwood C, Wilson M, McGowan $\mathrm{J}$, et al. Rapid review methods more challenging during COVID-19: commentary with a focus on 8 knowledge synthesis steps. Journal of Clinical Epidemiology 2020;126:177-83. https://doi.org/10.1016/j. jclinepi.2020.06.029
29. Burke NN, Galvin S, Devane D, Keenan C. COVID-19 Emergency Evidence Response Service: report from Ireland. In: Collaborating in response to COVID-19: editorial and methods initiatives across Cochrane. Cochrane Database of Systematic Reviews 2020;(12 Suppl 1). https://doi.org/10.1002/14651858.CD202002 


\title{
Developing rapid reviews of diagnostic tests in the time of COVID-19: current knowledge and future steps
}

\author{
Authors: Ingrid Arevalo-Rodriguez ${ }^{1}$, Andrea C Tricco ${ }^{2,3,4}$, Barbara Nussbaumer-Streit ${ }^{5}$, Karen R Steingart ${ }^{6}$, \\ David Kaunelis ${ }^{7}$, Pablo Alonso-Coello ${ }^{8,9}$, Patrick M Bossuyt ${ }^{10}$, Javier Zamora ${ }^{1}$ \\ Participating groups: Cochrane Screening and Diagnostic Tests Methods Group, Cochrane Rapid Reviews \\ Methods Group, Cochrane Iberoamérica, Cochrane Madrid
}

Corresponding Author: Ingrid Arevalo-Rodriguez: ingrid.arevalo@salud.madrid.org

\section{Introduction and background}

The COVID-19 pandemic has created challenges for the development of knowledge synthesis and evidence-based decision making.[1] More than ever, syntheses of evidence need to be conducted in good time and efficiently, to inform decisionmakers promptly with the best available evidence.[2] The rapid review, broadly defined as a knowledge synthesis strategy using limited or accelerated methods to expedite the time required to obtain a conclusive answer, has a crucial role in summarizing the unprecedented amount of COVID-19-related data.[3-7] Common rapid review actions to enhance the timeliness of knowledge synthesis include: strategies to limit the review scope (narrow the scope); tailoring of systematic review steps (review shortcuts); actions to increase resources in selected review processes (parallelization of tasks); and use of new technologies (automation). $[2,3,8]$ In March 2020, the Cochrane Rapid Review Methods group released interim guidance about how to conduct rapid reviews in the Cochrane evidence ecosystem and published the first rapid review about the effectiveness of quarantine measures to control the spread of COVID-19.[9-11]

Testing (e.g. for the presence of SARS-CoV-2 infection, the confirmation of COVID-19, or the response to a previous infection) is essential for controlling the COVID-19 pandemic, hence the importance of timely and comprehensive assessments of novel medical tests (12). COVID-19 diagnostic questions that have been addressed by rapid reviews include the accuracy of tests for detecting SARS-CoV-2 infection,[13] the role of COVID-19 serological tests,[14] the recommended criteria for discharge or de-isolation,[15] and the adequacy of different commercial kits and sampling of SARS-CoV-2 specimens.[16]

While methods for conducting full systematic reviews of diagnostic tests are well defined,[17] the methods for performing diagnostic rapid reviews are currently extrapolated from rapid reviews of effectiveness and safety.[3,7,18,19,20] However, the synthesis of diagnostic evidence comes with unique challenges given the fundamental differences between the methods used to summarize the evidence for interventions and those for diagnostic evidence.[17,21,22] Since 2018 we have been exploring the challenges of developing rapid reviews of diagnostic evidence. Here we summarize our most recent insights in the field, as well as anticipate further actions still needed to provide structured methodological guidance for developing rapid syntheses of diagnostic research findings.

\section{Key activities and strategies; outcomes and impact of activities}

As a baseline assessment of common practices, we examined the methodological characteristics of published diagnostic rapid reviews by scrutinizing repositories of Health Technology Assessment (HTA) agencies, as well as available publications in indexed journals.[23] After assessing 191 rapid reviews developed in 15 countries on four continents, we found suboptimal reporting of the methodological steps involved in the review production. For those areas with adequate reporting, we observed that most rapid reviews were broad in scope and assessed multiple outcomes and test applications. In addition, we found that well-known

${ }^{1}$ Hospital Universitario Ramón y Cajal, CIBER Epidemiology and Public Health, Spain; ${ }^{2}$ Li Ka Shing Knowledge Institute, St Michael's Hospital, Canada; ${ }^{3}$ University of Toronto, Canada; ${ }^{4}$ Queen's Collaboration for Health Care Quality, Queen's University, Canada; ${ }^{5}$ Cochrane Austria, Danube University Krems, Austria; ${ }^{6}$ Liverpool School of Tropical Medicine, UK; ${ }^{7}$ Canadian Agency for Drugs and Technologies in Health (CADTH), Canada; ${ }^{8} \mid$ beroamerican Cochrane Center-Servicio de Epidemiología Clínica y Salud Pública, Biomedical Research Institute (IIB-Sant Pau), Spain; ${ }^{\circ} \mathrm{CIBER}$ of Epidemiology and Public Health, Spain; ${ }^{10}$ University of Amsterdam, The Netherlands 
methodological tailoring strategies, such as limiting literature search by date or by the number of databases accessed, were rarely used.[23] The strategies more frequently reported included the narrative synthesis of findings ( $96 \%$ of rapid reviews), the omission of the assessment of the certainty of the evidence (92\%), the assessment of a single test (55\%) and the limitation of searches by language (43\%). See Figure 1.
After this initial exploration of methods, we carried out an international survey assessing current practice on diagnostic tests rapid reviews. [24] We surveyed 25 representatives of international agencies with previous experience in conducting rapid reviews of diagnostic issues. Our participants reported greater usage of limits in the scope than our previous scoping review suggested (Figure 1). Also, we found a high number of participants introducing limits
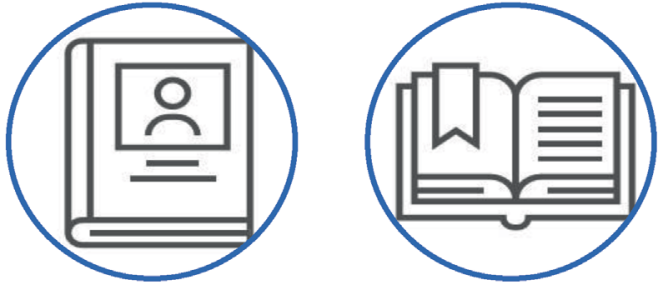

\section{General}

Development of a protocol (e.g. as an internal document) ${ }^{a}$

Use of a reporting template

Peer-review process (i.e. involving internal peers) ${ }^{\mathrm{a}}$

Public consultation of the draft review

Publication of the final review (e.g. in institutional repositories)

Team with high level of training (including experience in knowledge synthesis and diagnostic evidence) ${ }^{\mathrm{a}}$

Involvement of more than two review authors or more than one team (i.e. to address different types of evidence, to conduct stages in parallel)

\section{Stakeholder} involvement in review activities

\section{Narrowing the} scope

Defining a structured PICO questiona

Discussing the clinical pathway for the target condition (i.e. the role of the test in the current clinical practice, its intended application, and prior/alternative tests) ${ }^{a}$

Limiting the population $^{\text {a }}$

Limiting the number of index tests ${ }^{a, b}$

Limiting the number of comparisons $\mathrm{s}^{\mathrm{a}}$

Limiting the number of outcomes (e.g. accuracy only)

Limiting the number of applications of the tests (i.e. monitoring, screening, diagnosis) ${ }^{a}$
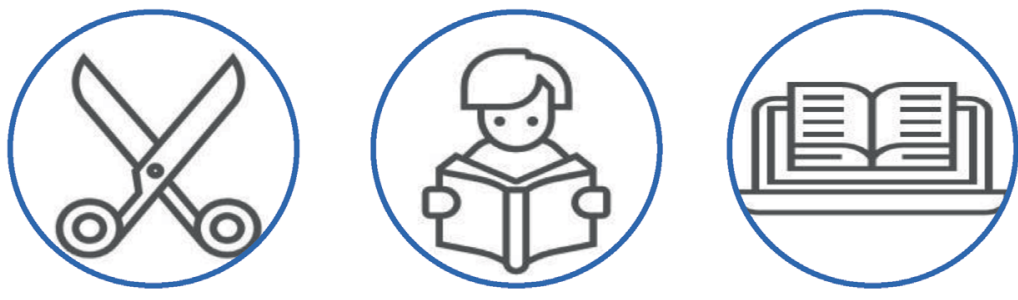

\section{Review shortcuts}

Using a previous review as a starting point (i.e. update of existing review, re-run search strategies) ${ }^{a}$

Limiting search strategies to one database, by language (e.g. English only) ${ }^{\mathrm{a}, \mathrm{b}}$ or by date ${ }^{a}$

Limiting the syntax of search strategies (e.g. focused subject

headings, terms in title only)

\section{Limiting search}

strategies' results using methodological filters ${ }^{a}$

Excluding additional searches (e.g. conference abstracts) ${ }^{a}$ Limiting screening of titles and abstracts and/or selection of full texts: one review author onlya

Limiting the data abstraction ${ }^{\mathrm{a}}$ and/or the quality appraisal: one review author only

Performing a narrative synthesis of findings $s^{a, b}$

Excluding a GRADE

assessment of findings $\mathrm{s}^{\mathrm{a}, \mathrm{b}}$

\section{Parallelization of tasks}

Multiple reviewe authors

completing the

eligibility

screening

Multiple review authors completing the data abstraction

Multiple review authors the quality appraisal completing

\section{Automation}

To assist in the screening/selection of references

To assist in the data abstraction

To assist in the quality appraisal

aMethod/strategy widely used by review developers.

${ }^{b}$ Method/strategy frequently reported in diagnostic rapid reviews.

Figure 1. Strategies and methods potentially useful during the development of rapid reviews about diagnostic issues 
on the search, including the use of methodological filters (56\%). We also confirmed that authors often used a narrative synthesis to describe their findings rather than formal meta-analysis.[23] Measures related to efficient use of resources for rapid review development included the involvement of highly trained staff (especially for small review teams), as well as the selection of studies and data abstraction being performed by a single review author. However, the use of automated approaches to speed up the review process was scarce (Figure 1 ).

\section{Lessons for the future: sustainability and transferability}

Our initial findings suggest that strategies focusing on limiting the review scope or using review shortcuts, or both, are more frequently adopted than strategies focusing on increasing the resources available for conducting the rapid review (parallelization of tasks and automation).[23,24] However, the levels of adoption of rapid review strategies, in general, is highly heterogeneous. Some review authors prefer to conduct all the standard review steps in a constrained timeframe (around two months, according to our scoping review). $[25,26]$ This approach poses an important challenge in the development of rapid reviews of diagnostic tests, due to recent explorations about the time required to complete a standard systematic review of diagnostic accuracy studies, which indicate that the probability of completing these reviews in 24 months is less than $10 \% .[27]$

The next steps of our research programme on diagnostic rapid reviews include:

- identifying the acceptability and potential challenges derived from the adoption of rapid review methods among experts in diagnostic evidence, knowledge synthesis of diagnostic evidence, and stakeholders requesting synthesis of diagnostic evidence;

- evaluating the usability and impact of rapid review strategies related to the efficient use of resources, such as parallelization of tasks, the involvement of highly trained reviewers, use of multiple reviewers in selected review tasks, and use of automated technologies to assist review processes;

- exploring the role of the stakeholder during the development of rapid reviews, given that these rapid reviews might be more relevant for decision-making in certain situations.[28-30]

In the near future, we intend to provide recommendations about optimal methods for rapid reviews of diagnostic evidence that will be of use for decision-making and policy development in future healthcare emergencies.

\section{Additional resources}

Rapid Diagnostic Reviews (D-RR): steps for performing a rapid review of diagnostic test accuracy studies (OSF profile): tinyurl.com/y3e4otak

\section{Acknowledgments}

Ingrid Arevalo-Rodriguez is funded by the Instituto de Salud Carlos III through the "Acción Estrategica en Salud 2013-2016/Contratos Sara Borrell convocatoria 2017/CD17/00219" (Co-funded by European Social Fund 2014-2020, "Investing in your future").

Andrea C Tricco is funded by a Tier 2 Canada Research Chair in Knowledge Synthesis.

Pablo Alonso-Coello is supported by a Miguel Servet investigator contract from the Instituto de Salud Carlos III (CPII15/0034).

\section{Declarations of interest}

$I A-R$ is a member of Cochrane Iberoamerica's Advisory Board and a member of the Cochrane Screening and Diagnostic Tests Methods Group. AT is a member of the Cochrane Equity Methods Group and the Cochrane Bias Methods Group. BN-S is Associate Director of Cochrane Austria, Convenor of the Cochrane Rapid Reviews Methods Group, and a member of the Advisory Boards of Cochrane Russia and Cochrane Sweden. KS is an Editor of Cochrane Infectious Diseases and a member of the Cochrane Screening and Diagnostic Tests Methods Group. PA-C is a Research Fellow with Cochrane Iberoamérica. PB is a member of the Cochrane Screening and Diagnostic Tests Methods Group and the Cochrane Prognosis Methods Group. JZ is Associate Director of Cochrane Madrid, an Advisory Board Member of Cochrane Iberoamerica, and a member of the Cochrane Screening and Diagnostic Tests Methods Group. The authors declare that they have no competing interests.

\section{Funding}

This study is funded by the Fundación para la Investigación Biomédica (FIBIO)-Hospital Universitario Ramon y Cajal (Internal grant IMP 1805/2018). The funder did not have a role in the design of the study and collection, analysis, interpretation of data, or in writing the final manuscript.

\section{References}

1. Wolkewitz M, Puljak L. Methodological challenges of analysing COVID-19 data during the pandemic. BMC Medical Research Methodology 2020;20:81. https://doi.org/10.1186/s12874-020-00972-6

2. Tricco AC, Garritty CM, Boulos L, Lockwood C, Wilson M, McGowan J, et al. Rapid review methods more challenging during COVID-19: commentary with a focus on 8 knowledge synthesis steps. Journal of Clinical Epidemiology 2020;126:177-83. https://doi.org/10.1016/j. jclinepi.2020.06.029

3. Tricco AC, Langlois EV, Straus SE, editors. Rapid reviews to strengthen health policy and systems: a practical guide. World Health Organization; 2017. Available from who.int/alliance-hpsr/resources/publications/ rapid-review-guide

4. Moher D, Stewart L, Shekelle P. All in the family: systematic reviews, rapid reviews, scoping reviews, realist reviews, and more. Systematic Reviews 2015;4:183. https://doi.org/10.1186/s13643-015-0163-7 
5. Hartling L, Guise JM, Kato E, Anderson J, Aronson N, Belinson S, et al. EPC methods: an exploration of methods and context for the production of rapid reviews. Research white paper. Rockville (MD): Agency for Healthcare Research and Quality; 2015. Available from effectivehealthcare.ahrq.gov/products/rapid-review-production/whitepaper

6. Khangura S, Konnyu K, Cushman R, Grimshaw J, Moher D. Evidence summaries: the evolution of a rapid review approach. Systematic Reviews 2012;1:10. https://doi.org/10.1186/2046-4053-1-10

7. Polisena J, Garritty C, Kamel C, Stevens A, Abou-Setta AM. Rapid review programs to support health care and policy decision making: a descriptive analysis of processes and methods. Systematic Reviews 2015;4:26. https://doi.org/10.1186/s13643-015-0022-6

8. Tricco AC, Antony J, Zarin W, Strifler L, Ghassemi M, Ivory J, et al. A scoping review of rapid review methods. BMC Medicine 2015;13:224. https://doi.org/10.1186/s12916-015-0465-6

9. Garritty C, Gartlehner G, Kamel C, King VJ, Nussbaumer-Streit B, Stevens A, et al. Interim guidance from the Cochrane Rapid Reviews Methods Group. March 2020. methods.cochrane.org/rapidreviews/sites/ methods.cochrane.org.rapidreviews/files/public/uploads/cochrane rr_-_guidance-23mar2020-v1.pdf

10. Garritty C, Gartlehner G, Nussbaumer-Streit B, King VJ, Kamel C, Stevens $A$, et al. Rapid review methods guidance aids in Cochrane's quick response to the COVID-19 crisis. In: Collaborating in response to COVID-19: editorial and methods initiatives across Cochrane. Cochrane Database of Systematic Reviews 2020;(12 Suppl 1). https://doi. org/10.1002/14651858.CD202002

11. Nussbaumer-Streit B, Mayr V, Dobrescu A, Chapman A, Persad $\mathrm{E}$, Klerings I, et al. Quarantine alone or in combination with other public health measures to control COVID-19: a rapid review. Cochrane Database of Systematic Reviews 2020;(4):CD013574. https://doi. org/10.1002/14651858.CD013574

12. Bossuyt PM. Testing COVID-19 tests faces methodological challenges. Journal of Clinical Epidemiology 2020;126:172-6. https://doi. org/10.1016/j.jclinepi.2020.06.037

13. Health Technology Wales. The clinical effectiveness of tests to detect the presence of SARS-CoV-2 virus, and antibodies to SARSCoV-2, to inform COVID-19 diagnosis Wales, UK; 2020. Available from healthtechnology.wales/reports-guidance/tests-to-inform-covid-19diagnosis

14. National Collaborating Centre for Methods and Tools. Rapid review: What serological tests are available, and what are their sensitivities and specificities? Canada: McMaster University; 29 May 2020. Available from nccmt.ca/uploads/media/media/0001/02/ d1db7fab4152258ca54fa634c2b49ac1d4efa585.pdf

15. COVID-19 Scientific Advisory Group. Which COVID-19 patients are safe to discharge from hospital?: a rapid response report. Canada: Alberta Health Services; 22 April 2020. Available from albertahealthservices.ca/assets/info/ppih/if-ppih-covid-19-sag-criteriafor-safe-discharge-from-hospital-rapid-review.pdf

16. COVID-19 Scientific Advisory Group. Clinical sensitivity and specificity of different COVID-19 testing kits. Canada: Alberta Health
Services; 15 April 2020. www.albertahealthservices.ca/assets/info/ppih/ if-ppih-covid-19-sag-comparison-of-testing-sites-rapid-review.pdf

17. Leeflang MM. Systematic reviews and meta-analyses of diagnostic test accuracy. Clinical Microbiology and Infection 2014;20(2):105-13. https://doi.org/10.1111/1469-0691.12474

18. Haby MM, Chapman E, Clark R, Barreto J, Reveiz L, Lavis JN. What are the best methodologies for rapid reviews of the research evidence for evidence-informed decision making in health policy and practice: a rapid review. Health Research Policy and Systems 2016;14:83. https://doi. org/10.1186/s12961-016-0155-7

19. Khangura S, Polisena J, Clifford TJ, Farrah K, Kamel C. Rapid review: an emerging approach to evidence synthesis in health technology assessment. International Journal of Technology Assessment in Health Care 2014;30:20-7. https://doi.org/10.1017/S0266462313000664

20. Pandor A, Kaltenthaler E, Martyn-St James M, Wong R, Cooper $\mathrm{K}$, Dimairo M, et al. Delphi consensus reached to produce a decision tool for SelecTing Approaches for Rapid Reviews (STARR). Journal of Clinical Epidemiology 2019;114:22-9. https://doi.org/10.1016/j. jclinepi.2019.06.005

21. Deeks J, Bossuyt P, Gatsonis CE. Cochrane handbook for systematic reviews of diagnostic test accuracy. The Cochrane Collaboration; 2010. Available from methods.cochrane.org/sdt/handbook-dta-reviews

22. Leeflang MM, Deeks JJ, Takwoingi Y, Macaskill P. Cochrane diagnostic test accuracy reviews. Systematic Reviews 2013;2:82. https:// doi.org/10.1186/2046-4053-2-82

23. Arevalo-Rodriguez I, Moreno-Nunez P, Nussbaumer-Streit B, Steingart K, Gonzalez Peña L, Buitrago-Garcia D, et al. Rapid reviews of medical tests used many similar methods to systematic reviews but key items were rarely reported: a scoping review. Journal of Clinical Epidemiology 2019;116:98-105. https://doi.org/10.1016/j. jclinepi.2019.09.004

24. Arevalo-Rodriguez I, Steingart KR, Tricco AC, Nussbaumer-Streit B, Kaunelis D, Alonso-Coello P, et al. Current methods for development of rapid reviews about diagnostic tests: an international survey. BMC Medical Research Methodology 2020;20:115. https://doi.org/10.1186/ s12874-020-01004-Z

25. Campbell F, Weeks L, Booth A, Kaunelis D, Smith A. A scoping review found increasing examples of rapid qualitative evidence syntheses and no methodological guidance. Journal of Clinical Epidemiology 2019;115:160-71. https://doi.org/10.1016/j.jclinepi.2019.05.032

26. Kelly SE, Moher D, Clifford TJ. Quality of conduct and reporting in rapid reviews: an exploration of compliance with PRISMA and AMSTAR guidelines. Systematic Reviews 2016;5:79. https://doi.org/10.1186/ s13643-016-0258-9

27. Beese S, Harris B, Davenport C, Mallet S, Takwoingi Y, Deeks JJ, editors. The first ten years of Cochrane DTA reviews: progress and common methodological challenges. Abstracts of the 25th Cochrane Colloquium; 2018; Edinburgh, UK: Cochrane Database of Systematic Reviews. Available from abstracts.cochrane.org/2018-edinburgh/ first-ten-years-cochrane-dta-reviews-progress-and-commonmethodological-challenges 
28. Wilson MG, Lavis JN, Gauvin FP. Developing a rapid-response program for health system decision-makers in Canada: findings from an issue brief and stakeholder dialogue. Systematic Reviews 2015;4:25. https://doi.org/10.1186/s13643-015-0009-3

29. Moore G, Redman S, Rudge S, Haynes A. Do policy-makers find commissioned rapid reviews useful? Health Research Policy and Systems 2018;16:17. https://doi.org/10.1186/s12961-018-0293-1
30. Hartling L, Guise JM, Hempel S, Featherstone R, Mitchell MD, Motu'apuaka ML, et al. Fit for purpose: perspectives on rapid reviews from end-user interviews. Systematic Reviews 2017;6:32. https://doi. org/10.1186/s13643-017-0425-7 


\title{
Rapid development of the Cochrane COVID-19 Study Register to support review production
}

\author{
Authors: Robin Featherstone ${ }^{1}$, Anna Last ${ }^{2}$, Lorne Becker ${ }^{3}$, Chris Mavergames ${ }^{4}$ \\ Participating groups: Cochrane Central Executive Team, Cochrane Australia, Cochrane France, Cochrane \\ Screening and Diagnostic Tests Methods Group
}

Corresponding Author: Robin Featherstone: rfeatherstone@cochrane.org

\section{Introduction and background}

In early 2020, an explosion in publishing about COVID-19 prompted an urgent need for an information system to support health researchers and Cochrane authors producing evidence syntheses. In March 2020, a cross-departmental and inter-organizational project team undertook the development of the Cochrane COVID-19 Study Register (CCSR) (covid-19.cochrane.org), an open-access search portal of COVID-19 primary study references.

\section{Key activities and strategies}

The CCSR project team started work in March 2020 on four key activities to launch the register:

\section{Deployment of a study-based register in the Cochrane Register of Studies (CRS)}

To facilitate agile product development of the CCSR, we used pre-existing Cochrane information systems wherever feasible. Cochrane's partner, Metaxis, built the CCSR within the Cochrane Register of Studies (CRS; community.cochrane.org/help/ tools-and-software/crs-cochrane-register-studies), a records management system and data repository currently used for the maintenance of the Cochrane Review Groups' registers and publication of the Cochrane Central Register of Controlled Trials (CENTRAL; cochranelibrary.com/central/about-central). But unlike most CRS registers that contribute to CENTRAL, Cochrane designed the CCSR to be 'study-based'. Building on a longstanding idea of linking 'threaded publications',[1] studybased registers add efficiency to review production and reduce the time needed to combine different references for the same studies.[2]

2. Creating a search protocol to identify COVID-19 references As a responsive information product based on community demand for emerging evidence, the CCSR was not restricted to randomized controlled trials as are many pre-existing registers in the CRS. The CCSR's search protocol was informed by Cochrane's question bank on COVID-19 (covidreviews.cochrane.org)[3] and designed to support a wide-range of reviews of different study design, including interventional, observational, diagnostic, prognostic, epidemiological, qualitative and economic designs. An information specialist designed sensitive search strategies to retrieve all eligible human studies on COVID-19 and expert searchers external to the project peer-reviewed the strategies. [4] Experienced Cochrane information specialists then executed daily or weekly searches, screened the results, linked threaded publications for the same studies, and classified the references in CRS.

3. Developing a domain-specific extension to the Cochrane PICO ontology and annotating references

To enable discovery and assist with study evaluation, Cochrane enhanced the CCSR's metadata by annotating references with terms for the studies' populations, interventions, comparators and outcomes (PICO). The Linked Data programme developed the Cochrane PICO Ontology and Vocabulary to describe Cochrane Reviews and included studies. The Cochrane Vocabulary links to existing health vocabularies, including $\mathrm{MeSH}$, SNOMED-CT, MedDRA, RxNorm, and ATC, supporting data re-use and semantic standardization across health and social care informatics. The vocabulary is currently searchable through PICO Search ${ }^{\text {BETA }}$ in the Cochrane Library (www.cochranelibrary. com/en/advanced-search/pico).

Given that COVID-19 was a novel topic area, Cochrane extended the vocabulary's subject domain to annotate the CCSR's studies by referencing other emerging COVID-19 terminologies, (e.g. SNOMED CT COVID-19 content and the WHO research priorities). To enable efficient annotation of the CCSR's references, Metaxis integrated the PICO Annotator, a tool developed for the Linked Data Project,[5] into CRS for use by experienced annotators from Cochrane's Information Specialist community. The screened, described and PICO-enhanced CCSR's study references

${ }^{1}$ Cochrane Central Executive, Canada; ${ }^{2}$ Cochrane Central Executive, UK; ${ }^{3}$ Cochrane Central Executive, USA; ${ }^{4}$ Cochrane Central Executive, Germany 
were then published online on a search portal developed by Cochrane's partner, Data Language.

\section{Publishing annotated references to an open access search portal}

For the open access search portal, Data Language created a new codebase built on existing Linked Data platform microservices and Application Programming Interfaces (APIs).[6] Data Language leveraged existing APIs and PICO data architecture to develop a rapid publishing platform for the register's records, while the new codebase supported features like combined PICOs and full-reference searches.

On 1 April 2020, fewer than three weeks after development began, Cochrane launched the public web application of the
CCSR with 868 references at covid-19.cochrane.org/. Since then, daily publication of new references to the search portal has been supported by ongoing study identification and annotation. As of 24 September 2020, Cochrane had published 21,417 references to the register.

\section{Outcomes and impact of activities}

The CCSR received over 10,000 users in its first month and continues to support approximately 10,000 monthly sessions.[7] As of 24 September, the register has been cited as a study source in 12 published Cochrane systematic reviews on COVID-19, three published Cochrane protocols, and 21 non-Cochrane reviews (Table 1).

Table 1. COVID-19 publications citing the Cochrane COVID-19 Study Register (as of 24 September 2020)

\begin{tabular}{ll}
\hline Title & DOI/URL \\
\hline Adverse drug reactions in COVID-19 patients: protocol for a living & https://doi.org/https://doi.org/10.21203/rs.3.rs-30471/v1
\end{tabular}

systematic review [Preprint]

Air pollution, SARS-CoV-2 transmission, and COVID-19 outcomes: a state- $\quad$ https://doi.org/10.1101/2020.08.16.20175901

of-the-science review of a rapidly evolving research area [Preprint]

Antibody tests for identification of current and past infection with SARS- $\quad$ https://doi.org/10.1002/14651858.CD013652

CoV-2 [Cochrane Review]

Antimicrobial mouthwashes (gargling) and nasal sprays administered to patients with suspected or confirmed COVID-19 infection to improve patient outcomes and to protect healthcare workers treating them

[Cochrane Review]

Antimicrobial mouthwashes (gargling) and nasal sprays to protect healthcare workers when undertaking aerosol-generating procedures (AGPs) on patients without suspected or confirmed COVID-19 infection [Cochrane Review]

Association between ethnicity and severe COVID-19 disease: a systematic review and meta-analysis [Preprint]

Bacille calmette-guerin (BCG) vaccine for preventing SARS-CoV-2 infection or improving COVID-19 outcome: evidence review of clinical benefits and harms

Chloroquine and hydroxychloroquine for prevention of COVID-19: evidence review of clinical benefits and harm

Chloroquine or hydroxychloroquine for prevention and treatment of COVID-19 [Cochrane Review]

Convalescent plasma or hyperimmune immunoglobulin for people with COVID-19: a living systematic review [Cochrane Review]

Current state of research about Chinese herbal medicines (CHM) for the treatment of coronavirus disease 2019 (COVID-19): a scoping review

The effect of funeral practices on bereaved friends and relatives' mental health and bereavement: implications for COVID-19

https://doi.org/10.1002/14651858.CD013627.pub2 https://doi.org/10.1002/14651858.CD013628.pub2 https://doi.org/10.1101/2020.08.12.20157271v1 infospace.mrc.ac.za/handle/11288/595256 infospace.mrc.ac.za/handle/11288/595264 https://doi.org/10.1002/14651858.CD013587 https://doi.org/10.1002/14651858.CD013600.pub2 https://doi.org/10.1089/acm.2020.0189 https://arc-w.nihr.ac.uk/covid-response/rapid-reports/ the-effect-of-funeral-practices-on-bereaved-friends-andrelatives-mental-health-and-bereavement-implicationsfor-covid-19/

Evidence summary: what is the utility of lung sonography in COVID-19 hdl.handle.net/10147/627790

pneumonia? 
Table 1. (continued)

\begin{tabular}{l} 
Title \\
\hline Digital contact tracing technologies in epidemics: a rapid review \\
[Cochrane Review] \\
\hline Feeding strategies to prevent neonatal SARS-CoV-2 infection in term or \\
late preterm babies born to mothers with confirmed COVID-19 \\
[Cochrane protocol] \\
\hline Hydroxychloroquine and chloroquine for survival in COVID-19: an \\
international collaborative meta-analysis of randomized trials [Protocol] \\
Imaging tests for the diagnosis of COVID-19 [Cochrane protocol] \\
\hline Interferon for COVID-19: evidence review of the clinical benefit and harm
\end{tabular}

\section{DOI/URL}

https://doi.org/10.1002/14651858.CD013699

https://doi.org/10.1002/14651858.CD013691

\section{osf.io/p2v84}

https://doi.org/10.1002/14651858.CD013639

knowledgehub.org.za/system/files/

elibdownloads/2020-08/Rapid\%20review\%20

of $\% 20$ Corticosteroids $\% 20$ for $\% 20$ COVID-19\%20

Update_6August2020.pdf

Interventions for treatment of COVID-19: a living systematic review with https://doi.org/10.1371/journal.pmed.1003293

meta-analyses and trial sequential analyses (The LIVING Project)

Interventions to reduce contaminated aerosols produced during dental

https://doi.org/10.1002/14651858.CD013686

procedures for preventing infectious diseases [Cochrane Review]

Is loss of sense of smell a diagnostic marker in COVID-19: a systematic

https://doi.org/10.1111/coa.13620

review and meta-analysis

Ivermectin for COVID-19: a living systematic review protocol 2020

[Protocol]

Janus associated kinases inhibitors for COVID-19 [Inibidores da janus

associated kinases para COVID-19]

Macrolide-clarithromycin task-force for the treatment and prophylaxis of COVID-19 as a single agent [Preprint]

Optimal delivery management for the prevention of early neonatal SARS-

CoV-2 infection [Cochrane protocol]

Oxygen targets in the intensive care unit during mechanical ventilation for acute respiratory distress syndrome: a rapid review [Cochrane Review]

Quarantine alone or in combination with other public health measures to control COVID-19: a rapid review [Cochrane Review]

Rapid, point-of-care antigen and molecular-based tests for diagnosis of SARS-CoV-2 infection [Cochrane Review]

Rapid synthesis of evidence on settings which have been associated with SARSCoV-2 transmission clusters 2020

https://doi.org/10.31219/osf.io/xsgke

oxfordbrazilebm.com/index.php/2020/04/14/inibidoresdas-janus-associated-kinases-para-covid-19/

https://doi.org/10.22541/au.158921784.48426659

https://doi.org/10.1002/14651858.CD013689

https://doi.org/10.1002/14651858.CD013708

https://doi.org/10.1002/14651858.CD013574.pub2

Multisystem inflammatory syndrome in children related to COVID-19: a systematic review [Preprint]

SARS-CoV-2 seroprevalence studies: a rapid review [Preprint]

https://doi.org/10.1002/14651858.CD013705

Signs and symptoms to determine if a patient presenting in primary care or hospital outpatient settings has COVID-19 disease [Cochrane Review]

Travel-related control measures to contain the COVID-19 pandemic: a rapid review [Cochrane Review]

Universal screening for SARS-CoV-2 infection: a rapid review [Cochrane Review]

Use of antimicrobial mouthwashes (gargling) and nasal sprays by healthcare workers to protect them when treating patients with suspected or confirmed superspreadingdatabase.github.io/Evidence_on_clusters_final. pdf

https://doi.org/10.1101/2020.08.17.20173641

https://doi.org/10.21203/rs.3.rs-56014/v1

https://doi.org/10.1002/14651858.CD013665

https://doi.org/10.1002/14651858.CD013717

COVID-19 infection [Cochrane Review]

The vaccine journey for COVID-19: a comprehensive systematic review of current clinical trials in humans

https://doi.org/10.1002/14651858.CD013718

https://doi.org/10.1002/14651858.CD013626.pub2

https://doi.org/10.23736/s0031-0808.20.03958-0 
In addition to contributing to systematic review production by Cochrane and other review teams, the CCSR contributes to living guidelines by the Australian National COVID-19 Clinical Evidence Taskforce (covid19evidence.net.au),[8] and is currently recommended as a COVID-19 evidence source by Guidelines International Network (GIN).[9] As featured in the Tech for Good series by New Digital Age, the CCSR was recommended as a "precisely scoped, authoritative source of the latest clinical evidence on COVID-19 from around the world".[10]

\section{Lessons for the future: sustainability and transferability}

Like CENTRAL, the CCSR is a centrally organized, collaborative partnership between Cochrane Departments, Review Groups and developers. Recent experience building CENTRAL in CRS with automated data feeds, machine learning and Crowd screening informs our future planning for the CCSR.[11]

To ensure sustainability of the CCSR, Cochrane is automating the register's searches, working with partners to validate machine learning algorithms to determine study eligibility, and building community engagement through our citizen science platform, Cochrane Crowd (crowd.cochrane.org), to help screen and describe potentially eligible studies for the CCSR.

Beyond COVID-19, the CCSR will help Cochrane prepare for future public health emergencies of international concern where we can use the same approach to build responsive study registers on emerging health topic areas of urgent global interest.

\section{Additional resources}

- Cochrane COVID-19 Study Register: covid-19.cochrane.org

- Cochrane Linked Data: linkeddata.cochrane.org

- Cochrane Ontologies: data.cochrane.org/ontologies

\section{Acknowledgements}

The authors acknowledge Cochrane's partners Metaxis and Data Language for developing and maintaining the Cochrane COVID-19 Study Register's (CCSR) data systems and web-based applications. We acknowledge the CCSR's Centralised Search Team (Deirdre Beecher, Samantha Cox, Candida Fenton, Anne Littlewood, Anna Noel-Storr, Joanne Platt, Susanna Wisniewski), COVID-19 Study Annotators from the Cochrane Information Specialists' community and Cochrane Crowd contributors who were integral to identifying and classifying study references for the register. The authors acknowledge the peer-reviewers of the CCSR's search strategies, Douglas Salzwedel and Julie Glanville. Finally, we acknowledge our advisors from Cochrane's Geographic Centres and Review Groups, in particular Steve McDonald, Jac Dinnes, René Spijker and Isabelle Boutron for providing feedback on the register's search protocol, initial sensitivity evaluation and our future development plans.

\section{Declarations of interest}

The authors declare that they have no competing interests. RF, AL and CM are Cochrane employees. LB is a Cochrane consultant.

\section{Funding}

This project was funded by Cochrane.

\section{References}

1. Chalmers I, Altman DG. How can medical journals help prevent poor medical research? Some opportunities presented by electronic publishing. Lancet 1999;353(9151):490-3. https://doi.org/10.1016/S01406736(98)07618-1

2. Shokraneh F, Adams CE. Study-based registers of randomized controlled trials: starting a systematic review with data extraction or meta-analysis. Biolmpacts 2017;7(4):209-17. https://doi.org/10.15171/ bi. 2017.25

3. Kew K, De Haan S, Foxlee R, Marshall R. Prioritization during a pandemic: taking a strategic approach for a sustained response. In: Collaborating in response to COVID-19: editorial and methods initiatives across Cochrane. Cochrane Database of Systematic Reviews 2020;(12 Suppl 1). https://doi.org/10.1002/14651858.CD202002

4. Cochrane. About COVID-19 Study Register. community.cochrane.org/ about-covid-19-study-register (accessed 10 September 2020).

5. Mavergames C, Beecher D, Becker LA, Last A, Ali A. Cochrane's Linked Data Project: how it can advance our understanding of surrogate endpoints. Journal of Law, Medicine \& Ethics 2019;47(3):374-80. https:// doi.org/10.1177/1073110519876166

6. Shearer M. Launching the Cochrane COVID-19 Study Register. datalanguage.com/news/launching-the-cochrane-covid-19-studyregister (accessed 7 September 2020).

7. Cochrane. More than 10,000 users in its first month: Cochrane's COVID-19 Study Register supports rapid evidence synthesis in the global challenge to combat COVID-19. 22 May 2020. cochrane.org/news/ more-10000-users-its-first-month-cochranes-covid-19-study-registersupports-rapid-evidence

8. National COVID-19 Clinical Evidence Taskforce. Australian guidelines for the clinical care of people with COVID-19. 2020 [version 20]. covid19evidence.net.au (accessed 7 September 2020).

9. Guidelines International Network. COVID-19 Evidence Resources. g-i-n.net/covid-19/covid-19-evidence-resources (accessed 7 September 2020).

10. Oakes A. Tech for good: Cochrane COVID-19 Study Register. New Digital Age, 29 April 2020. newdigitalage.co/2020/04/29/tech-for-goodcochrane-covid-19-register

11. Noel-Storr AH, Dooley G, Wisniewski S, Glanville J, Thomas J, Cox $S$, et al. Cochrane Centralised Search Service showed high sensitivity identifying randomized controlled trials: a retrospective analysis. Journal of Clinical Epidemiology 2020;Aug 13. https://doi.org/10.1016/j. jclinepi.2020.08.008 


\title{
Learning from emergency trauma teams:
} an organizational approach for conducting (very) rapid reviews

\author{
Authors: Gerald Gartlehner ${ }^{1,2}$, Barbara Nussbaumer-Streit ${ }^{1}$ \\ Participating groups: Cochrane Austria \\ Corresponding Author: Gerald Gartlehner: gartlehner@cochrane.at
}

\section{Introduction and background}

The coronavirus disease 2019 (COVID-19) pandemic has again underscored the shortcomings of systematic reviews to address the urgent needs of decision makers during health crises. [1] Even a month after the World Health Organization (WHO) had declared the COVID-19 outbreak a public health emergency of international concern,[2] only four systematic reviews relevant to COVID-19 topics had been published globally in English.[3] By the time publications of systematic reviews increased, major decisions about lockdowns, school closings, travel restrictions and quarantine measures had already been made.

By providing answers to time-critical health issues within days or weeks, rapid reviews have emerged as a new form of evidence synthesis.[4-7] Rapid reviews employ systematic methods but streamline or omit certain methodological steps that systematic reviews use. Because they are subject to time pressure, rapid reviews face substantial challenges regarding team organization and quality assurance.

In late January 2020, WHO notified Cochrane Austria, as a WHO Collaborating Centre, that it would commission a rapid review on a yet unknown COVID-19 topic with a seven-day turnaround. During the lead-time before the start of the project, we developed an organizational approach to counteract two of our main concerns regarding the seven-day timeline:

1. the quick turnaround time would increase the risk for errors which could compromise the quality of the review;

2. required preparatory steps (like pilot testing a data extraction form) could slow down the review team and reduce efficiency.

Following, we briefly describe the roles and processes we adapted to address these issues.

\section{Key activities and strategies}

WHO commissioned a review on the effectiveness of quarantine to control COVID-19. Our rapid review team consisted of seven investigators, one information specialist, an organizational assistant, and two medical students. To minimize the risk for errors, we organized the leadership positions of the rapid review team in a way that mimicked those of an emergency trauma team.

Well-organized trauma teams work with clearly defined roles to maximize quality of care in critical situations characterized by time pressure. Decision-making and quality assurance are assigned to two different individuals who collaborate closely, namely the team leader and a senior person who supports the team leader. Trauma team leaders direct and co-ordinate all activities, motivate the team, and create a positive working atmosphere.[8] They are the focal point of communication, make the main decisions but otherwise have a mostly hands-off role during acute patient care.

A crucial position is the team leader support, a senior colleague who takes on a quality-assurance role by standing a few steps behind the trauma team and observing decisions and processes. Through situational awareness, the team leader support can identify potential or actual errors and anticipate difficulties. With the team leader support person conducting oversight, the team leader is free to focus on individual decisions of patient care.

For our rapid review, the team leader had a mostly conventional role but with less operational involvement than usual. He drafted the protocol, assigned roles, participated in every step of the review, communicated with team members, led daily meetings, answered review related questions, and made all major decisions.

The team leader support was a newly introduced role. The team leader support was a senior investigator who also had the expertise 
to serve as a team leader. She was involved in each step of the review but mainly as an observer in a quality-assurance capacity. By having the team leader support also act as the point person for communication with stakeholders, the team leader could utilize valuable time to focus exclusively on the review process. The team leader support co-drafted the protocol and checked its completeness, discussed conflicts of screening decisions during daily team meetings, spot-checked data extraction accuracy, and confirmed that the review process adhered to the guidance of the Cochrane Rapid Review Methods Group.[9] In addition, she vetted certainty of evidence ratings, and prepared the report outline and the draft version of the Introduction and Methods sections of the report during the still ongoing review process.

We used web-based systematic review software (Covidence),[10] and a web-based collaborative platform (Microsoft Teams),[11] for every step of the review.

The biggest difference to our standard rapid review process involved ensuring that the team leader and team leader support always remained a step ahead of the main review team. For example, while the main team screened abstracts, the team leader and team leader support started on full-text screening. This included the preparation and piloting of the full-text review form, defining exclusion criteria, and then initiating full-text screening. All the while, they remained cognizant of potential challenges.
Once abstract screening was completed, the main team could seamlessly proceed to full-text screening. At this point, the team leader and team leader support progressed to the next review stage and started piloting risk of bias and data extraction forms. The team leader and team leader support in the end synthesized the evidence and assessed the certainty of evidence. Figure 1 outlines the adapted review process.

We also made other minor adaptations to the usual review process.

- We negotiated with WHO that the clock for the seven-day turnaround started ticking after topic refinement with WHO had been completed.

- The team piloted abstract screening while the literature searches were still in progress. By the time literature searches were completed, the team was ready to start abstract screening.

- One or two daily team meetings facilitated communication by setting new goal markers, resolving questions, and clarifying misunderstandings.

- All team members focused solely on the rapid review and put aside all other project obligations during the rapid review week.

\section{Outcomes and impact of activities}

Within seven days we screened 2620 abstracts and 109 full-text articles, of which 29 met inclusion criteria.

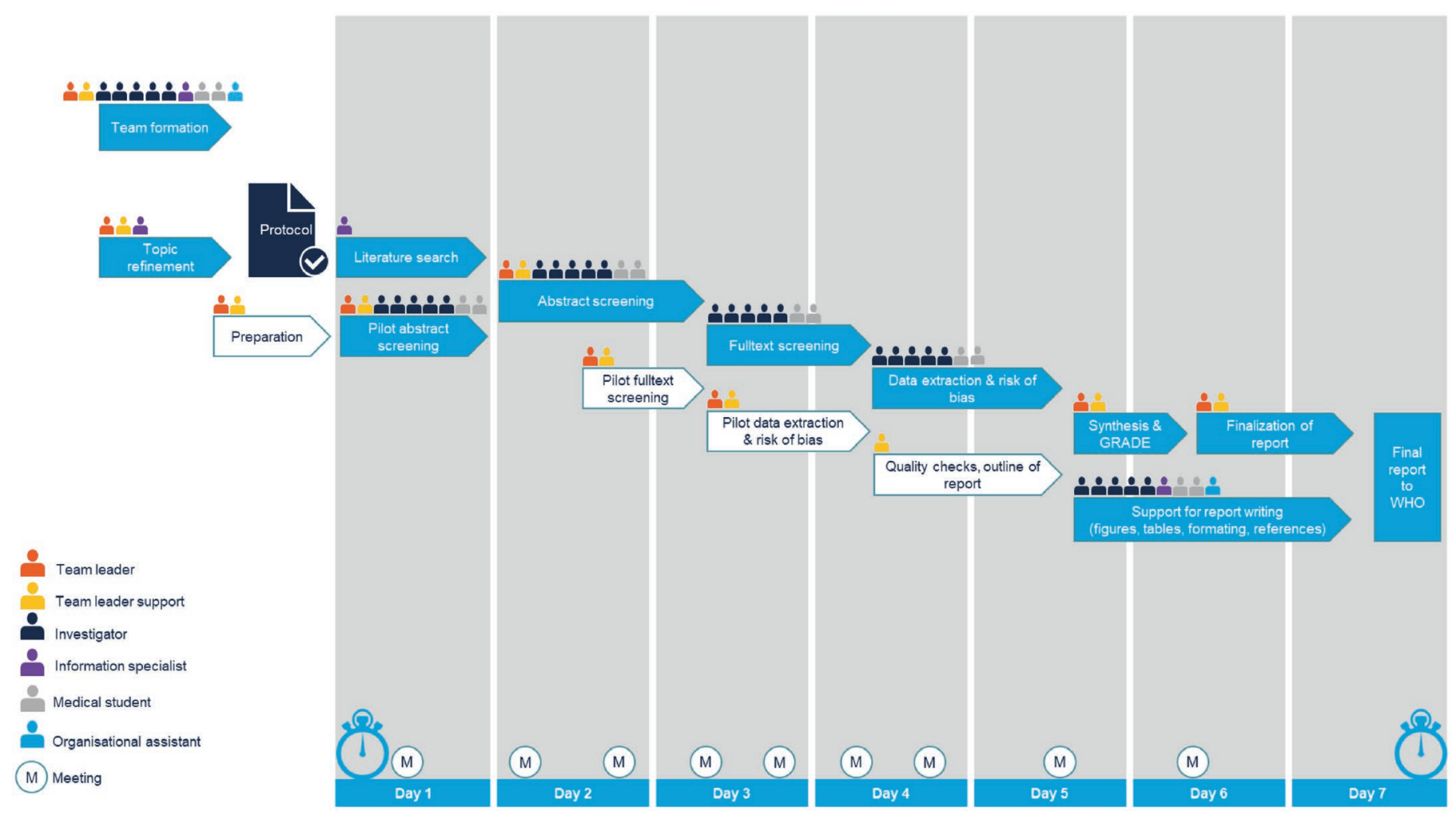

Figure 1. Flow of the adapted rapid review process 
The newly introduced position of the team leader support was an invaluable asset for the production of a high-quality review in such an abbreviated time period. Team leader support added a layer of quality assurance crucial to ensuring the efficiency and coordination of work under time pressure. Keeping the team leader a step ahead of the main team, while challenging, allowed the review to progress smoothly.

An important aspect for completing a rapid review on such a complex topic within seven days is the commitment of the team. Our team was willing to work in the evenings and during the weekend, which helped to meet the tight timeline. Because the strain on the team is high, to maintain motivation and quality, such reviews should be the exception rather than the rule.

Our streamlined process enabled us to submit our rapid review on the effectiveness of quarantine to control COVID-19 to WHO on time. After an update, the report was published as the first Cochrane rapid review on the Cochrane Library.[12]

\section{Lessons for the future: sustainability and transferability}

With one investigator focusing exclusively on quality assurance, the one-week production of a high-quality rapid review is possible, if the team focuses entirely on the review. Streamlining the review process by always keeping the team leader a step ahead of the main team, while challenging, is crucial to such a review's overall success.

\section{Declarations of interest}

GG is Director of Cochrane Austria and Convenor of the Cochrane Rapid Reviews Methods Group. BN-S is Associate Director of Cochrane Austria and Convenor of the Cochrane Rapid Reviews Methods Group. The authors declare no other competing interests.

\section{Funding}

None

\section{References}

1. World Health Organization. Report of the WHO-China Joint Mission on Coronavirus Disease 2019 (COVID-19). February 2020. who.int/docs/ default-source/coronaviruse/who-china-joint-mission-on-covid-19final-report.pdf
2. World Health Organization. Rolling updates on coronavirus disease (COVID-19). who.int/emergencies/diseases/novel-coronavirus-2019/ events-as-they-happen (accessed 13 July 2020).

3. Evidence Synthesis Program. COVID-19 Evidence Reviews. covid19reviews.org (accessed 28 June 2020).

4. Moore G, Redman S, Rudge S, Haynes A. Do policy-makers find commissioned rapid reviews useful? Health Research Policy and Systems 2018;16(1):17. https://doi.org/10.1186/s12961-018-0293-1

5. Mijumbi-Deve R, Rosenbaum SE, Oxman AD, Lavis JN, Sewankambo NK. Policymaker experiences with rapid response briefs to address health-system and technology questions in Uganda. Health Research Policy and Systems 2017;15(1):37. https://doi.org/10.1186/s12961-0170200-1

6. Hartling L, Guise J-M, Hempel S, Featherstone R, Mitchell MD, Motu'apuaka ML, et al. Fit for purpose: perspectives on rapid reviews from end-user interviews. Systematic Reviews 2017;6(1):32. https://doi. org/10.1186/s13643-017-0425-7

7. Peterson K, Floyd N, Ferguson L, Christensen V, Helfand M. User survey finds rapid evidence reviews increased uptake of evidence by Veterans Health Administration leadership to inform fast-paced healthsystem decision-making. Systematic Reviews 2016;5(1):132. https://doi. org/10.1186/s13643-016-0306-5

8. Courtenay M, Nancarrow S, Dawson D. Interprofessional teamwork in the trauma setting: a scoping review. Human Resources for Health 2013;11(1):57. https://doi.org/10.1186/1478-4491-11-57

9. Garritty C, Gartlehner G, Kamel C, King VJ, Nussbaumer-Streit B, Stevens A, et al. Cochrane Rapid Reviews: interim guidance from the Cochrane Rapid Reviews Methods Group. March 2020. Available from methods.cochrane.org/rapidreviews/cochrane-rr-methods

10. Covidence. Version accessed April 2020. Melbourne, Australia: Veritas Health Innovation. Available at covidence.org.

11. MS Teams. Microsoft Corporation, Redmond, USA. Version accessed after April 2020. Available at microsoft.com/microsoft-365/microsoftteams

12. Nussbaumer-Streit B, Mayr V, Dobrescu Al, Chapman A, Persad $\mathrm{E}$, Klerings I, et al. Quarantine alone or in combination with other public health measures to control COVID-19: a rapid review. Cochrane Database of Systematic Reviews 2020;(4):CD013574. https://doi. org/10.1002/14651858.CD013574 


\title{
Fostering international co-operation and junior researchers' engagement during a pandemic: InterNetCOVID-19
}

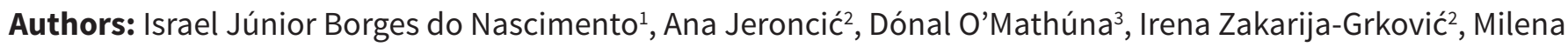 \\ Soriano Marcolino ${ }^{1}$, Thilo Caspar von Groote ${ }^{4}$, Tina Poklepović-Peričić ${ }^{2}$
}

Participating groups: Cochrane Croatia, Cochrane Sweden, Cochrane Brazil, Cochrane US Network, Cochrane Iran

Corresponding Author: Israel Júnior Borges do Nascimento: israeljbn@ufmg.br

\section{Introduction and background}

This report reflects on the activities of an international and multidisciplinary collaboration that began in February 2020 as the full impact of the novel coronavirus (SARS-CoV-2) was not yet realized. Prior to the implementation of social distancing and travel restrictions, researchers from different parts of the world were seeking opportunities to collaborate and contribute to the medical field with high-quality research. With the impending pandemic looming, and COVID-19 publications starting to appear, a Cochrane International Mobility program placement took place at Cochrane Sweden and Cochrane Croatia.[1] During the visits, Israel Júnior Borges do Nascimento conceived the idea of creating an international collaboration aimed at contributing to knowledge on this emerging disease by rapidly providing comprehensive, high-quality evidence summaries. The challenge of how to gather contributors quickly, who could rapidly collate, assess, and synthesize the available evidence, was daunting.

\section{Key activities and strategies}

As the pandemic spread, publications about COVID-19 rapidly started to appear, mostly as consecutive case reports. Many reported COVID-19 symptoms and potential laboratory and radiological disease descriptors on small numbers of cases. Combining information from them was difficult due to their heterogeneity. In addition, studies on pharmacological and nonpharmacological interventions were lacking at the time. To ensure a high-quality systematic review, a team of researchers with different expertise and from different fields, but with available time, and willingness to contribute, was needed. Cochrane TaskExchange (taskexchange.cochrane.org) gave us the mechanism to gather such people and also to establish closer international professional ties.
In early 2020 we put two posts on TaskExchange seeking researchers interested in collaborating on a systematic review of COVID-19. Applicants were asked to describe their experience, resilience, personal sources of inspiration, and why they felt they were a good match for this project. While these questions might be seen as 'less academic' and 'more personal', we sought to value 'being' more than 'having', believing that junior researchers might not have an extraordinary history of publications, but would have stamina and rigour for the long haul. In addition, we invited a few senior researchers to join, based on their expertise.

The International Task Force Network of Coronavirus Disease 2019 (InterNetCOVID-19) was formed!

The collaboration worked well by respecting and balancing the energy of early-stage researchers who pushed ambitiously for very comprehensive analyses and embraced the suggestions and expertise of senior researchers. Being a large group, we were able to check and recheck data collection and extraction and deliver quality reviews while respecting each other and learning together.

\section{Outcomes and impact of activities}

Our recruitment strategy led to more than 50 applications from around the world within 10 days. Based on their interests in the topic and answers to the questions, we selected 11 candidates. As the work expanded, the Task Force grew to include 35 members. Work began in February 2020 on a protocol for a scoping review of case studies.[2] The group worked day and night (having different time zones working in our favour), resulting in the scoping review with meta-analysis being published on 30 March 2020.[3] As of 20 September 2020, the paper has been cited over 150 times (Google Scholar), achieved a high Attention Score (48) in Altmetric, and

${ }^{1}$ Federal University of Minas Gerais, Brazil; ${ }^{2}$ Cochrane Croatia, University of Split School of Medicine, Croatia; ${ }^{3}$ The Ohio State University, USA; ${ }^{4}$ University of Münster, Germany 
informed a clinical guideline on acute and subacute pulmonary rehabilitation.[4,5] Furthermore, the scoping review was used in establishing the clinical management protocol for patients suspected of having COVID-19 in federal hospitals in Brazil.[6]

This first experience built the team's confidence and encouraged us to continue working together. We revised our scoping review methods and conducted an updated review of newly published studies, this time with a comprehensive analysis including more than 50 outcomes. This was published in September 2020.[7] Spurred on by the enthusiasm of the whole team, and because of the growing number of systematic reviews of COVID-19 data, we undertook an overview of published systematic reviews, which is currently under peer review.[8] Overall, the three reviews aimed to provide a clear presentation of the most up-to-date and reliable information that would either assist stakeholders making timely decisions or guide researchers worldwide in designing future projects.

In just over six months, our team has submitted three manuscripts to peer-reviewed journals. Yet, the most important outcomes are not quantifiable. Team members are exploring collaborations on projects unrelated to COVID-19 as strong research bonds have formed. The creation of an international scientific network, where senior researchers work side-by-side with young researchers, embracing differences and enabling people to apply previous knowledge as well as to develop new skills, is immeasurable. By leading the collaboration and being the true driving forces, young researchers showed themselves to be reliable, resilient and diligent workers. By seeking out input from more experienced researchers, they are building leadership competencies and soft skills.

\section{Lessons for the future: sustainability and transferability}

Working with a large group of people is challenging, especially when people come from different backgrounds, cultures, and professions, and have different languages and time zones. Given the importance of timely publication of scientific evidence during pandemics, maintaining the same level of rigour was a challenge. Questions arose over specific methodological details, leading to requests for assistance from experienced Cochrane authors. In all cases, timely help was provided. Other challenges arose with team dynamics. Everyone got tired at different points or needed to focus on other professional or personal matters. Because of this, encouragement was needed at various times to complete certain tasks. This was done respectfully through inspiring and motivational messages, through instant messaging applications, and spurring people on to achieve the team's agreed goals. Sometimes, however, misunderstandings arose over how much work a person would perform, some disagreements occurred over how best to do specific tasks, or difficulties arose over the best balance between working rapidly or meticulously. While such challenges are inherent to any all-inclusive, fast-paced, interdisciplinary collaboration, the patience and mutual respect learned within Cochrane helped immensely. Our experience showcases Cochrane's continuing collaborative ethos.

\section{Additional resources}

- Cochrane International Mobility: Israel Júnior Borges do Nascimento

- Cochrane Croatia: Cochrane International Mobility

- Abstract: Cochrane TaskExchange experience during the coronavirus pandemic

\section{Acknowledgments}

A special message of gratitude to Dr Matteo Bruschettini (Cochrane Sweden), who enabled the overseas exchange experience of the first author. We also would like to thank Maria Björklund (Cochrane Sweden) who ran the searches in record time. Lastly, but equally important, we thank all InterNetCOVID-19 members, without whom these results would not have been achieved.

\section{Declarations of interest}

AJ, IZ-G and TP-P are members of Cochrane Croatia. DO'M is a member of the Executive Committee of the Cochrane US Network. All authors declare no other competing interests.

\section{Funding}

We acknowledge support from the Open Access Publication Fund of the University of Münster and from The Ohio State University.

\section{References}

1. Cochrane. Cochrane International Mobility. training.cochrane.org/ cochrane-international-mobility

2. Borges do Nascimento IJ, Marcolino MS, Bragazzi NL, Bjorklund M, Jayarajah U, Esfahani MA, et al. The novel 2019 coronavirus (nCoV) infection in humans: a systematic review protocol. PROSPERO 2020; CRD42020170623 www.crd.york.ac.uk/prospero/display_record. php?ID=CRD42020170623

3. Borges do Nascimento IJ, Cacic N, Abdulazeem HM, von Groote TC, Jayarajah U, Weerasekara I, et al. Novel coronavirus infection (COVID-19) in humans: a scoping review and meta-analysis. Journal of Clinical Medicine 2020;9(4):941. https://doi.org/10.3390/jcm9040941

4. Kurtaiş AY, Köseoğlu BF, Taşkıran ÖÖ, Ordu-Gökkaya NK, Delialioğlu SÜ, Tur BS, et al. Pulmonary rehabilitation principles in SARSCOV-2 infection (COVID-19): a guideline for the acute and subacute rehabilitation. Turkish Journal of Physical Medicine and Rehabilitation 2020;66(2):104-20. https://doi.org/10.5606/tftrd.2020.6444

5. Altmetric: Novel coronavirus infection (COVID-19) in humans: a scoping review and meta-analysis. altmetric.com/details/78645288 (accessed 22 September 2020).

6. Marcolino MS, Figueiredo NM, Sousa e Silva MV, Resende RE. Manejo do paciente com suspeita de COVID-19 em serviço de urgência e unidade de terapia intensiva [Clinical management of suspected cases of COVID-19 in emergency departments and intensive care unit]. Centro 
de Telessaúde HC-UFMG. 25 May 2020. Available from https://tinyurl. com/y56q9jmy

7. Borges do Nascimento IJ, Von Groote TC, O’Mathúna DP, Abdulazeem HM, Henderson C, Jayarajah U, et al. Clinical, laboratory and radiological characteristics and outcomes of novel coronavirus (SARS-CoV-2) infection in humans: a systematic review and series of meta-analyses. PLOS One 2020;15(9):e0239235. http://doi.org/10.1371/ journal.pone.0239235

8. Borges do Nascimento IJ, O'Mathúna DP, von Groote TC, Abdulazeem HM, Weerasekara I, Marusic A, et al. Coronavirus disease (COVID-19) pandemic: an overview of systematic reviews. MedRxiv 2020;April 22. https://doi.org/10.1101/2020.04.16.20068213 


\title{
The Central Editorial Service: a collaborative editorial process for publishing high-priority Cochrane Reviews
}

\author{
Authors: Helen Wakeford ${ }^{1}$, Clare Dooley ${ }^{2}$, Anne-Marie Stephani ${ }^{1}$, Rachel Marshall ${ }^{1}$, Robin Featherstone ${ }^{3}$, Deirdre \\ Walshe ${ }^{4}$, Denise Mitchell ${ }^{1}$, Leticia Rodrigues ${ }^{1}$, Toby Lasserson ${ }^{1}$
}

Participating groups: Cochrane Central Executive Team, Cochrane Public Health Hub, Cochrane Review Groups

Corresponding Author: Helen Wakeford: hwakeford@cochrane.org

\section{Introduction and background}

The onset of the COVID-19 pandemic saw systematic review teams coming together to synthesize emerging evidence on SARS-CoV-2 treatment, transmission prevention, prognosis and diagnosis. To react quickly and inform decision-making, Cochrane needed to expedite the publication of priority COVID-19 reviews and their updates, whilst ensuring high standards and rigorous process. Cochrane's Central Editorial Service (community.cochrane.org/ review-production/production-resources/cochranes-centraleditorial-service; formerly the Fast-Track Service) was able to work collaboratively to establish an accelerated editorial process. We have facilitated the publication of 17 COVID-19 reviews and updates in under six months. A further 18 COVID-19 reviews are currently in the editorial process or pending submission.

\section{Key activities and strategies}

The Central Editorial Service core team consists of Managing Editors, administrative support, an Information Specialist, a Plain Language Summary (PLS) writer and copy-editor, with the Editorial Service Lead and Deputy Editor in Chief of Cochrane providing management and strategic oversight, respectively. The team works with Cochrane Review Groups (CRGs), Network Editors, and the Cochrane Methods Support Unit (MSU; methods.cochrane.org/ about-us/cochrane-central-executive-methods-team/methodssupport-unit) to take reviews from submission to publication. Early in the pandemic we worked with Cochrane's Methods team, Informatics \& Technology Services (ITS), and Rapid Review Methods Group (methods.cochrane.org/rapidreviews) to enable Cochrane to publish rapid reviews as a new review type,[1] and we created a dedicated COVID-19 editorial system using ScholarOne Manuscripts. Reviews can be submitted in Word in a flexible format, facilitating submissions from authors who are not familiar with Cochrane's processes. Where possible, editorial tasks are performed in parallel rather than sequentially, to reduce the time to publication without compromising editorial rigour (Figure 1). While we focus on invited submissions in Cochrane's COVID-19 priority areas, we also accept speculative submissions.[2]

Submitted manuscripts are reviewed by a Managing and Associate Editor. The MSU supports specific statistical queries or novel methods, and an Information Specialist reviews the search methods. Suitability for external peer review is established within the first few days of submission and methodological review is completed within one week.

In parallel, the Central Editorial Service relies on close collaboration with CRGs. When the CRG has not been involved in authoring a review, CRG editors may comment on the review, recommend external peer reviewers and approve the final manuscript for publication.

Specialist external peer reviewers are identified and agreed in advance of submission of the review, enabling the peer review process to be completed within one week. We seek consumer input for all reviews via the COVID-19 consumer rapid response group. [3]

The Public Health Hub was formed as a 'one-stop-shop' for methods and content review of COVID-19 public health reviews submitted to the Central Editorial Service. It includes members of Cochrane Public Heath, a statistician from the MSU, and the Senior Editor for Cochrane Public Health and Health Systems.[4]

The Central Editorial Service benefits from dedicated copy-editing support. Sections of COVID-19 reviews that are unlikely to change in

${ }^{1}$ Cochrane Central Executive Team, UK; ${ }^{2}$ Cochrane Central Executive Team, Australia; ${ }^{3}$ Cochrane Central Executive Team, Canada;

${ }^{4}$ Cochrane Infectious Diseases, UK 


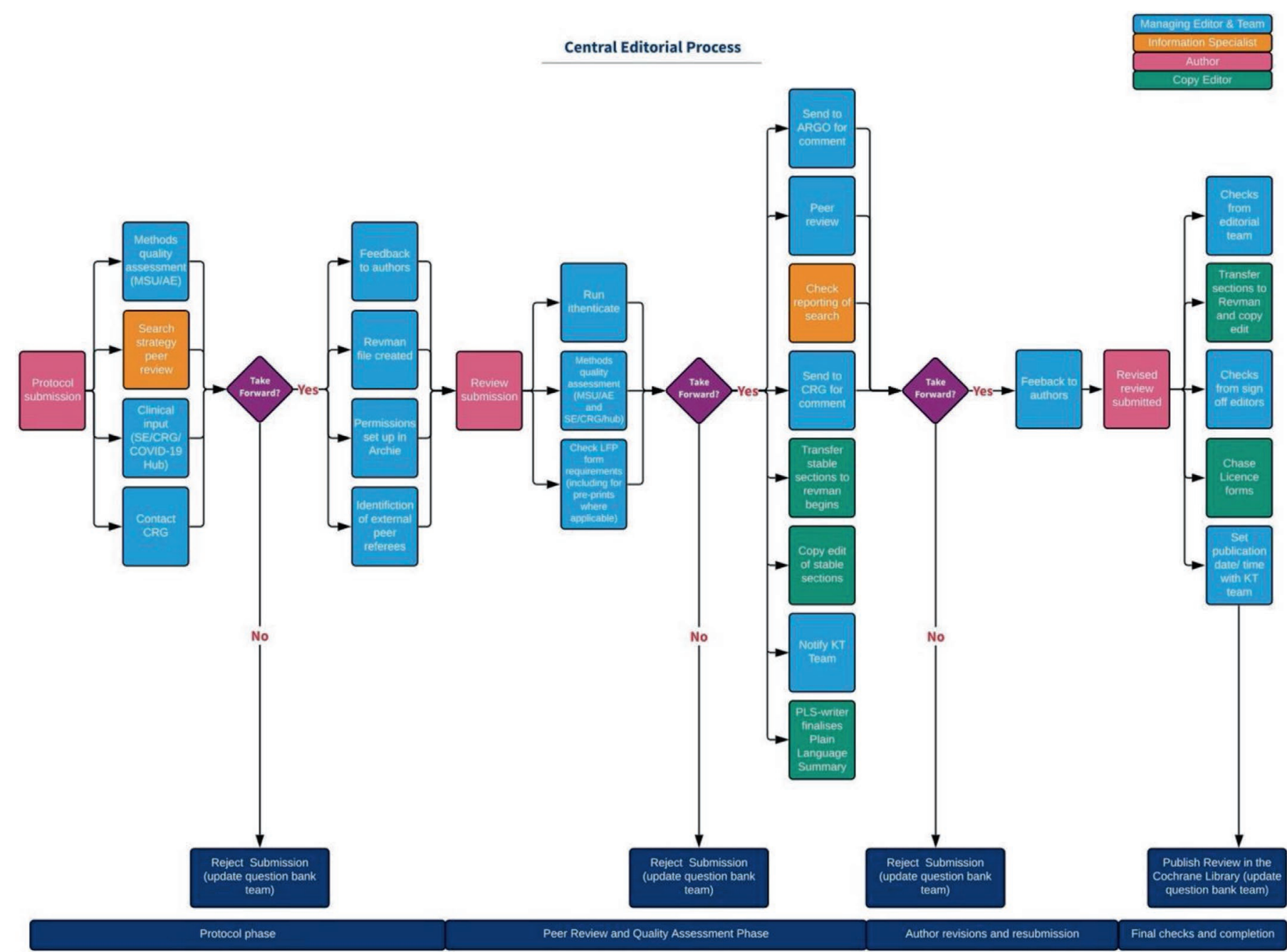

Figure 1. Cochrane central editorial process

response to peer review (e.g. references, appendices, characteristics of studies tables) are copy-edited in parallel to peer review to expedite the editorial process. A dedicated PLS writer ensures that the PLS is accessible.

To ensure a smooth publication process and optimal dissemination, the Central Editorial Service co-ordinates publication of all COVID-19 reviews with Cochrane's publishing and Knowledge Translation teams.

\section{Outcomes and impact of activities}

The ability to publish priority COVID-19 reviews in a flexible and expedited manner has been an important part of Cochrane's responsiveness and reflexivity to the COVID-19 pandemic.

Between April and September 2020, the Central Editorial Service received 78 submissions. Working with CRGs and teams across Cochrane, 29 were rejected on quality and priority grounds, 14 were delegated to CRGs, 18 are pending submission to the Central Editorial Service or in the editorial process, and 17 have been published.

Published reviews span multiple priority areas including transmission control,[5-9] infection prevention,[10-12] diagnosis,[13-16] treatment,[17-20] and interventions to address social isolation.[21]

The mean time from review submission to publication was 27 days, with a range of 11 to 49 days. Most published within one month of submission (10/17). The majority were commissioned by guideline developers, including several by the World Health Organization. $[6,7,13-16]$

The close collaboration between the Central Editorial Service and Cochrane's Knowledge Translation Department meant that reviews were widely disseminated, including via two press briefings with the Science Media Centre (sciencemediacentre.org). The Central 
Editorial Service managed the editorial process for the three most viewed reviews in the Cochrane Library in 2020.[7,12,13] At the time of writing, two of these reviews feature in the 99th percentile of Altmetric scores (altmetric.com), and the top 5\% of all research outputs ever tracked by Altmetric. $[7,13]$

\section{Lessons for the future: sustainability and transferability}

We have built on the existing Central Editorial Service processes to deliver an expedited, collaborative editorial process for highpriority COVID-19 reviews. Working with CRGs, Networks, the MSU and KT has enabled Cochrane to oversee the production of 17 high-quality and relevant COVID-19 reviews. This has contributed to Cochrane's rapid and reflexive response to the pandemic and has enabled Cochrane to make a substantive contribution to the COVID-19 evidence base. There is no sign of submissions slowing, with 18 reviews pending submission or in the editorial process at the time of writing.

While some of the tight timelines achieved in the early stages of the COVID-19 pandemic may be difficult to sustain in the long term, the parallel processes, collaborative structure and core team that have been established means that this model can be utilized for other high-priority Cochrane reviews going forward, COVID-19-related or otherwise.

\section{Acknowledgements}

We are very grateful to Associate Editors Liz Bickerdike, Sarah Hodgkinson, Nuala Livingstone, Jen Hilgart, and Rachel Richardson, who have provided invaluable comments on many of the COVID-19 reviews published via the Central Editorial Service. We also thank the Methods Support Unit, led by Kerry Dwan, for methodological guidance. We thank Kayleigh Kew and the Cochrane Methods team for managing the COVID-19 prioritization process and submissions to the Central Editorial Service. Thank you to Monaz Mehta and Katie Abbotts for their efforts in publishing and dissemination. We are grateful to Mike Brown, Harald Harkner, and Paul Garner for their editorial input and sign-off on several of these reviews, and to the Public Health Hub (Lisa Bero, Luke Wolfenden, Andy Anglemyer, and Jodie Doyle) for their insightful feedback on all COVID-19 public health reviews submitted to the Central Editorial Service. We acknowledge the contribution of Ruth Foxlee and Douglas Salzwedel who reviewed the search methods of submitted protocols and reviews. We thank Therese Docherty for her contribution to project management, and we are grateful to Richard Morley for convening the COVID-19 consumer rapid response group, which we have utilized for consumer reviews of all our COVID-19 reviews.

\section{Declarations of interest}

HW, CD, A-MS, RM, RF, DM, LR, and TL are employees of the Editorial and Methods Department in Cochrane's Central Executive Team. DW is supported by the Research, Evidence and Development Initiative (READIt). READ-It (project number 300342-104) is funded by UK aid from the UK government; however, the views expressed do not necessarily reflect the UK government's official policies.

\section{Funding}

Cochrane

\section{References}

1. Garritty C, Gartlehner G, Nussbaumer-Streit B, King VJ, Kamel C, Stevens A, et al. Rapid review methods guidance aids in Cochrane's quick response to the COVID-19 crisis. In: Collaborating in response to COVID-19: editorial and methods initiatives across Cochrane. Cochrane Database of Systematic Reviews 2020;(12 Suppl 1). https://doi. org/10.1002/14651858.CD202002

2. Kew K, De Haan S, Foxlee R, Marshall R. Prioritization during a pandemic: taking a strategic approach for a sustained response. In: Collaborating in response to COVID-19: editorial and methods initiatives across Cochrane. Cochrane Database of Systematic Reviews 2020;(12 Suppl 1). https://doi.org/10.1002/14651858.CD202002

3. Morley R, Crowe S, Haddaway N, Lytvyn L, Marshall C, Marshall $\mathrm{R}$, et al. Cochrane's COVID-19 consumer rapid response group. In: Collaborating in response to COVID-19: editorial and methods initiatives across Cochrane. Cochrane Database of Systematic Reviews 2020;(12 Suppl 1). https://doi.org/10.1002/14651858.CD202002

4. Bero LA, Wolfenden L, Doyle J, Anglemyer A. The Public Health Hub: an adaptive model for rapid publication of high-priority reviews. In: Collaborating in response to COVID-19: editorial and methods initiatives across Cochrane. Cochrane Database of Systematic Reviews 2020;(12 Suppl 1). https://doi.org/10.1002/14651858.CD202002

5. Anglemyer A, Moore TH, Parker L, Chambers T, Grady A, Chiu K, et al. Digital contact tracing technologies in epidemics: a rapid review. Cochrane Database of Systematic Reviews 2020;(8):CD013699. https:// doi.org/10.1002/14651858.CD013699

6. Burns J, Movsisyan A, Stratil JM, Coenen M, Emmert-Fees KM, Geffert $\mathrm{K}$, et al. Travel-related control measures to contain the COVID-19 pandemic: a rapid review. Cochrane Database of Systematic Reviews 2020;(9):CD013717. https://doi.org/10.1002/14651858.CD013717

7. Nussbaumer-Streit B, Mayr V, Dobrescu Al, Chapman A, Persad E, Klerings I, et al. Quarantine alone or in combination with other public health measures to control COVID-19: a rapid review. Cochrane Database of Systematic Reviews 2020;(4):CD013574. https://doi. org/10.1002/14651858.CD013574

8. Nussbaumer-Streit B, Mayr V, Dobrescu Al, Chapman A, Persad $\mathrm{E}$, Klerings I, et al. Quarantine alone or in combination with other public health measures to control COVID-19: a rapid review. Cochrane Database of Systematic Reviews 2020;(9):CD013574. https://doi. org/10.1002/14651858.CD013574.pub2

9. Viswanathan M, Kahwati L, Jahn B, Giger K, Dobrescu Al, Hill C, et al. Universal screening for SARS-CoV-2 infection: a rapid review. Cochrane Database of Systematic Reviews 2020;(9):CD013718. https://doi. org/10.1002/14651858.CD013718

10. Houghton C, Meskell P, Delaney H, Smalle M, Glenton C, Booth A, et al. Barriers and facilitators to healthcare workers' adherence with infection prevention and control (IPC) guidelines for respiratory infectious diseases: a rapid qualitative evidence synthesis. Cochrane Database of Systematic Reviews 2020;(4):CD013582. https://doi. org/10.1002/14651858.CD013582 
11. Paludan-Müller AS, Boesen K, Klerings I, Jørgensen KJ, Munkholm $K$. Hand cleaning with ash for reducing the spread of viral and bacterial infections: a rapid review. Cochrane Database of Systematic Reviews 2020;(4):CD013597. https://doi.org/10.1002/14651858.CD013597

12. Verbeek JH, Rajamaki B, Ijaz S, Sauni R, Toomey E, Blackwood B, et al. Personal protective equipment for preventing highly infectious diseases due to exposure to contaminated body fluids in healthcare staff. Cochrane Database of Systematic Reviews 2020;(5):CD011621. https://doi.org/10.1002/14651858.CD011621.pub5

13. Deeks JJ, Dinnes J, Takwoingi Y, Davenport C, Spijker R, TaylorPhillips S, et al. Antibody tests for identification of current and past infection with SARS-CoV-2. Cochrane Database of Systematic Reviews 2020;(6):CD013652. https://doi.org/10.1002/14651858.CD013652

14. Dinnes J, Deeks JJ, Adriano A, Berhane S, Davenport C, Dittrich S, et al. Rapid, point-of-care antigen and molecular-based tests for diagnosis of SARS-CoV-2 infection. Cochrane Database of Systematic Reviews 2020;(8):CD013705. https://doi.org/10.1002/14651858.CD013705

15. Salameh J-P, Leeflang MM, Hooft L, Islam N, McGrath TA, Van der Pol CB, et al. Thoracic imaging tests for the diagnosis of COVID-19. Cochrane Database of Systematic Reviews 2020;(9):CD013639. https:// doi.org/10.1002/14651858.CD013639.pub2

16. Struyf T, Deeks JJ, Dinnes J, Takwoingi Y, Davenport C, Leeflang $\mathrm{MM}$, et al. Signs and symptoms to determine if a patient presenting in primary care or hospital outpatient settings has COVID-19 disease.
Cochrane Database of Systematic Reviews 2020;(7):CD013665. https:// doi.org/10.1002/14651858.CD013665

17. Flumignan RL, Tinôco JD, Pascoal PI, Areias LL, Cossi MS, Fernandes $\mathrm{MI}$, et al. Prophylactic anticoagulants for patients hospitalised with COVID-19. Cochrane Database of Systematic Reviews 2020;(9):CD013739. https://doi.org/10.1002/14651858.CD013739

18. Valk SJ, Piechotta V, Chai KL, Doree C, Monsef I, Wood EM, et al. Convalescent plasma or hyperimmune immunoglobulin for people with COVID-19: a rapid review. Cochrane Database of Systematic Reviews 2020;(5):CD013600. https://doi.org/10.1002/14651858.CD013600

19. Piechotta V, Chai KL, Valk SJ, Doree C, Monsef I, Wood EM, et al. Convalescent plasma or hyperimmune immunoglobulin for people with COVID-19: a living systematic review. Cochrane Database of Systematic Reviews 2020;(7):CD013600. https://doi.org10.1002/14651858.CD013600. pub2

20. Chai KL, Valk SJ, Piechotta V, Kimber C, Monsef I, Doree C, et al. Convalescent plasma or hyperimmune immunoglobulin for people with COVID-19: a living systematic review. Cochrane Database of Systematic Reviews 2020;(10):CD013600. https://doi.org/10.1002/14651858. CD013600.pub2

21. Noone C, McSharry J, Smalle M, Burns A, Dwan K, Devane D, et al. Video calls for reducing social isolation and loneliness in older people: a rapid review. Cochrane Database of Systematic Reviews 2020;(5):CD013632. https://doi.org/10.1002/14651858.CD013632 


\title{
The Public Health Hub: an adaptive model for rapid publication of high-priority reviews
}

\author{
Authors: Lisa Beroํㅡ, Luke Wolfenden², Jodie Doyle², Andrew Anglemyer ${ }^{4}$ \\ Participating groups: Cochrane Public Health and Health Systems \\ Corresponding Author: Lisa Bero: lisa.bero@CUAnschutz.edu
}

\section{Introduction and background}

Cochrane's COVID-19 response has included the rapid publication of relevant Cochrane reviews, updates, and rapid reviews. Historically, very rapid publication of quality reviews has posed a challenge for Cochrane due to overstretched review group editorial teams, lack of funding for editorial staff, delays due to editorial and peer review processes and slow responses from author teams. Publication of COVID-19 reviews presents additional challenges as many important review questions during initial phases of the outbreak required synthesis of studies employing research designs not traditionally included in Cochrane reviews, such as modelling studies. Furthermore, the assessment and processing of COVID-19 reviews required diverse and specialist expertise. Additional challenges to rapid review production during COVID-19 have been acknowledged.[1]

\section{Key activities and strategies}

As Cochrane gathered high-priority COVID-19-related questions from stakeholders, [2] it quickly became apparent that many of the questions were about public health measures for the detection and prevention of infection. There was a need for a number of published reviews that would fall within the scope of the Public Health and Health Systems (PHHS) Network (publichealth.cochrane.org). The PHHS Network includes the Public Health (ph.cochrane. org), Infectious Diseases (cidg.cochrane.org), Effective Practice and Organisation of Care (epoc.cochrane.org), Consumers and Communication (cccrg.cochrane.org), Tobacco (tobacco.cochrane. org), and Work (work.cochrane.org) Cochrane Review Groups, enabling it to draw on a range of expertise pertinent to a number of priority review topics.

To facilitate publication of reviews through the Network, Cochrane formed a 'Public Health Hub' as an extension of the Central Editorial Service team (community.cochrane.org/review-production/ production-resources/cochranes-central-editorial-service).[3] The Hub includes members of the Central Editorial Service team, plus a methodologist, the Senior Editor of the PHHS Network, and a Coordinating Editor and Managing Editor of Cochrane Public Health. Cochrane methodologists developed abbreviated review proposal and protocol forms specifically for rapid reviews. [4] Hub members provided input about modifying these protocols for public health questions and non-randomized studies.

The Public Health Hub participates in vetting COVID-19 priority review topics, sometimes working with author teams to rapidly refine the review scope, question and methods. The Hub provides internal peer and methodological review of protocols and reviews, often within 48 hours. The timely internal review is facilitated by assignment of multiple editorial staff from the Central Editorial Service team and Cochrane Public Health to assess each review, enabling the review process to progress once 'sufficient' editorial assessment has occurred. Additionally, an editorial team situated across global time-zones enables review assessment and processing to occur continually. An important process for the rapid publication of high-quality reviews is the identification of external peer reviewers, including content area experts, in advance of receiving the protocols and reviews. To assist authors with making timely revisions, the Hub also provides guidance on how to respond to the peer-review comments. Regular bi-monthly meetings of the broader Hub members to discuss upcoming and ongoing reviews and to resolve any outstanding editorial issues has also been crucial to facilitate swift turnover times.

The Central Editorial Service co-ordinates Central Editorial Service team members of the Hub, also provide editing assistance, particularly for abstracts, 'Summary of findings' tables, and Plain language summaries, which are often poorly completed by author teams and a cause of publication delay. The majority of the five reviews seen by the Public Health Hub have been published through Cochrane Public Health. Communication within the Hub has been facilitated by the geographical proximity of three of the editors, who are able to participate in ad-hoc meetings outside of the bi-monthly 


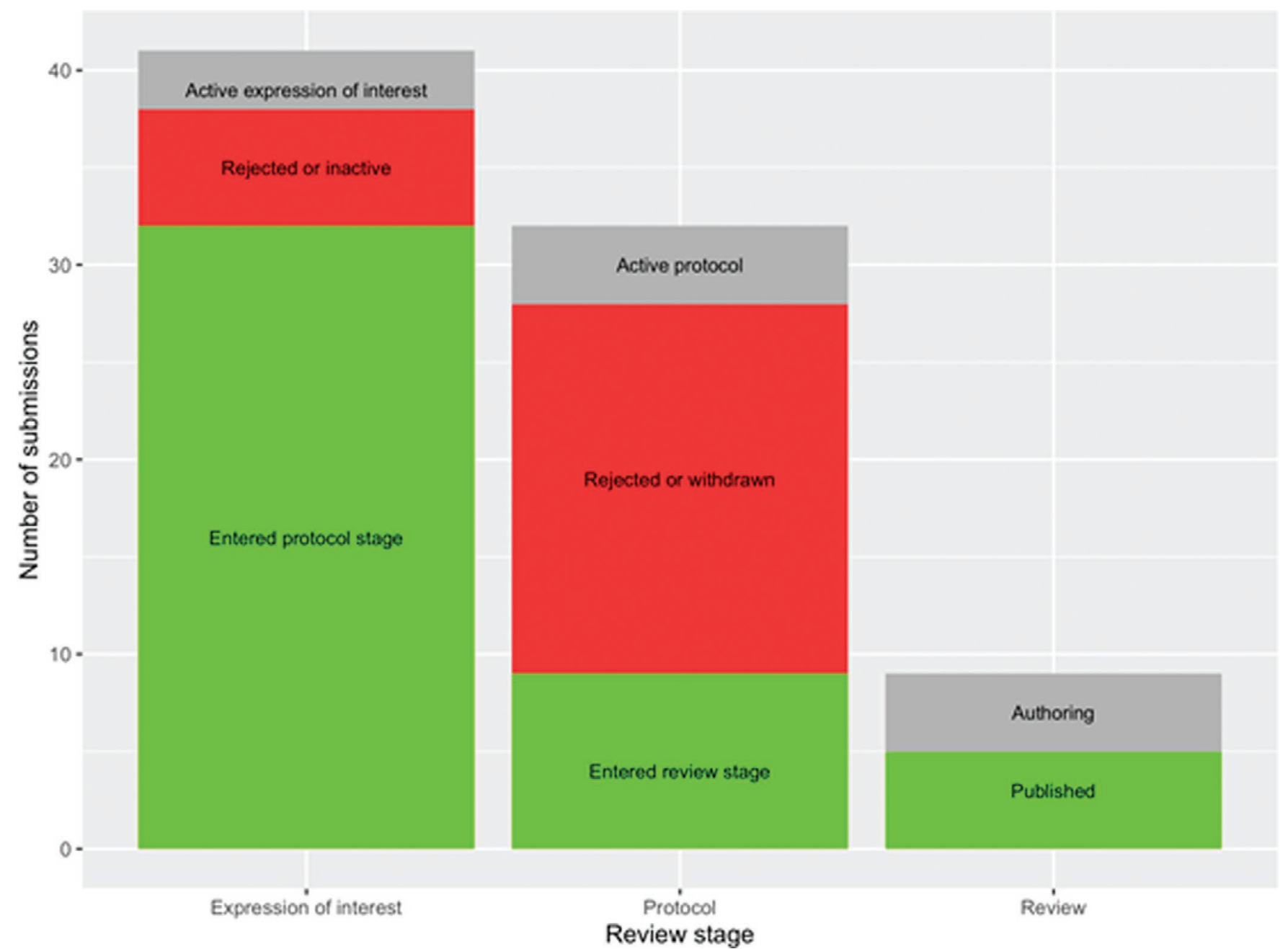

Figure 1. Outcomes of COVID-19 Cochrane review submissions with Public Health Hub involvement

broader Hub meetings when internal decisions needed to be reached quickly.

\section{Outcomes and impact of activities}

The Public Health Hub has leveraged the infrastructure of the PHHS Network and the Cochrane Central Editorial Service to reorientate editorial processes to assess more than 30 protocols, and publish five high-quality rapid reviews in just five months, with an additional four rapid reviews in their final stages of authoring (see Figure 1). Some of these reviews have also received substantial input from review groups within the PHHS Network, particularly Cochrane Infectious Diseases and Cochrane Work, and are published through these groups. These reviews evaluate evidence that is critical to making decisions about public health interventions such as quarantine,[2] contact tracing,[3] hand washing,[4] use of personal protective equipment,[5] and video calls for the elderly.[6] In addition, these reviews are being published much faster than the average year-long editorial process for a Cochrane review.
Given the policy importance of our COVID-19 public health reviews, it is not surprising that they have had high media attention. Using the Altmetric Attention Score comparing research outputs with similar publication dates, all five published reviews scored higher than $97 \%$ of articles of similar age on average (ranging from $93^{\text {rd }}$ to $99^{\text {th }}$ percentile).

We have also received personal feedback regarding the usefulness and timeliness of our reviews. Susan Shaw, MD, FRCPC, Chief Medical Officer, Saskatchewan Health Authority has written to the Hub,

"It is great to have rapid reviews available within such times of rapid growth in information (and misinformation). I've been at times quite concerned with what at times seems like the early sharing of what is not very good quality data.

We are working hard to make good decisions informed by good data and information....Please know that your work is making a difference and is being translated into action. 
I think the questions you have selected and the answers/guides that will come will really help the world be better prepared. Thank you."

\section{Lessons for the future: sustainability and transferability}

The Hub model seems a viable option for rapidly publishing Cochrane reviews on high-priority topics. It has demonstrated that providing central editorial support for publication of reviews facilitates the process. The Hub model also provided the flexibility to publish reviews on high-priority topics that were not currently on the agenda of a specific review group.

As shown in Figure 1, the Hub model revealed the importance of spending more time on developing the review question as a large proportion of topics and protocols were rejected early in the process. Collaboration of the Central Editorial Service with Network editors with public health expertise allowed us to make the reviews more relevant to a public health audience. By modifying the review protocol form and providing early feedback to authors on refining the question, we were able to avoid delays due to confusion about scope or inclusion criteria. Some review topics and protocols that were rejected early were reformulated using questions and methods that were more 'doable' for a rapid review. We also learned the value of peer-reviewer specialist expertise, even though it was sometimes challenging to access this expertise given it is in such high demand.

\section{Acknowledgements}

We thank Kayleigh Kew and Helen Wakeford for providing information on the flow of reviews through the Public Health Hub. We thank Toby Lasserson, Rachel Marshall, and Anne-Marie Stephani for their involvement in developing the Hub. We thank Susan Shaw for permission to quote from her email to Lisa Bero.

\section{Declarations of interest}

LB is Senior Editor for Cochrane Public Health and Health Systems and Senior Editor for Research Integrity; JD is Managing Editor of Cochrane Public Health; AA is Epidemiology Editor for the Cochrane Methods Support Unit, part of the Cochrane Central Executive. LW is Joint Co-ordinating Editor of Cochrane Public Health and an NHMRC Fellow at the University of Newcastle, Australia.

\section{Funding}

None

\section{References}

1. Tricco AC, Garritty CM, Boulos L, Lockwood C, Wilson M, McGowan J, et al. Rapid review methods more challenging during COVID-19: commentary with a focus on 8 knowledge synthesis steps. Journal of Clinical Epidemiology 2020; June 29. https://doi.org/10.1016/j. jclinepi.2020.06.029

2. Kew K, De Haan S, Foxlee R, Marshall R. Prioritization during a pandemic: taking a strategic approach for a sustained response. In: Collaborating in response to COVID-19: editorial and methods initiatives across Cochrane. Cochrane Database of Systematic Reviews 2020;(12 Suppl 1). https://doi.org/10.1002/14651858.CD202002

3. Wakeford H, Dooley C, Stephani A-M, Marshall R, Featherstone R, Walshe D, et al. The Central Editorial Service: a collaborative editorial process for publishing high-priority Cochrane reviews. In: Collaborating in response to COVID-19: editorial and methods initiatives across Cochrane. Cochrane Database of Systematic Reviews 2020;(12 Suppl 1). https://doi.org/10.1002/14651858.CD202002

4. Garritty C, Gartlehner G, Nussbaumer-Streit B, King VJ, Kamel C, Stevens A, et al. Rapid review methods guidance aids in Cochrane's quick response to the COVID-19 crisis. In: Collaborating in response to COVID-19: editorial and methods initiatives across Cochrane. Cochrane Database of Systematic Reviews 2020;(12 Suppl 1). https://doi. org/10.1002/14651858.CD202002

5. Nussbaumer-Streit B, Mayr V, Dobrescu A, Chapman A, Persad E, Klerings I, et al. Quarantine alone or in combination with other public health measures to control COVID-19: a rapid review. Cochrane Database of Systematic Reviews 2020;(4):CD013574. https://doi. org/10.1002/14651858.CD013574

6. Anglemyer A, Moore TH, Parker L, Chambers T, Grady A, Chiu K, et al. Digital contact tracing technologies in epidemics: a rapid review. Cochrane Database of Systematic Reviews 2020;(8):CD013699. https:// doi.org/10.1002/14651858.CD013699

7. Paludan-Müller AS, Boesen K, Klerings I, Jørgensen KJ, Munkholm K. Hand cleaning with ash for reducing the spread of viral and bacterial infections: a rapid review. Cochrane Database of Systematic Reviews 2020;(4):CD013597. https://doi.org/10.1002/14651858.CD013597

8. Verbeek JH, Rajamaki B, ljaz S, Sauni R, Toomey E, Blackwood B, et al. Personal protective equipment for preventing highly infectious diseases due to exposure to contaminated body fluids in healthcare staff. Cochrane Database of Systematic Reviews 2020;(5):CD011621. https:// doi.org/10.1002/14651858.CD011621.pub5

9. Noone C, McSharry J, Smalle M, Burns A, Dwan K, Devane D, et al. Video calls for reducing social isolation and loneliness in older people: a rapid review. Cochrane Database of Systematic Reviews 2020;(5):CD013632. https://doi.org/10.1002/14651858.CD013632 


\section{The eCOVID-19 living recommendations map and gateway to contextualisation}

Authors: Adrienne Stevens ${ }^{1 \star}$, Tamara Lotfi ${ }^{1 \star}$, Elie A. Akl ${ }^{2}$, Maicon Falavigna ${ }^{1,3,4}$, Tamara Kredo ${ }^{5}$, Joseph L. Mathew ${ }^{6}$, Marc T. Avey ${ }^{7}$, Romina Brignardello-Petersen ${ }^{1}$, Derek K. Chu ${ }^{1}$, Omar Dewidar ${ }^{8,9}$, Stephanie Duda ${ }^{1}$, Bart Diet ${ }^{10}$, Signe Flottorp ${ }^{11,12}$, Anisa Hajizadeh ${ }^{1}$, Alfonso Iorio ${ }^{1}$, Miloslav Klugar ${ }^{13,14,15}$, Miranda W. Langendam ${ }^{16}$, Justyna Litynska ${ }^{10}$, Lorenzo Moja ${ }^{17}$, Ignacio Neumann ${ }^{18}$, Artur Nowak ${ }^{10}$, Thomas Piggott ${ }^{1,19}$, Marge Reinap ${ }^{20}$, Karla Solo ${ }^{1}$, Maureen Smith $^{21}$, Peter Tugwell ${ }^{8,22}$, Alexis F. Turgeon ${ }^{23}$, Gunn Vist ${ }^{11}$, Vivian Welch ${ }^{8,9}$, James M. Wright ${ }^{24}$, Lara Al-Hakim², Chris Cotoi ${ }^{1}$, Marina Davoli ${ }^{25}$, Karin Dearness ${ }^{26}$, Elaine Harrow ${ }^{27}$, Lucia Kantorova ${ }^{13,14,15}$, Joanne P. Khabsa ${ }^{2}$, Jitka Klugarova ${ }^{13,15}$, Dominik Mertz ${ }^{1}$, Zachary Munn ${ }^{28}$, Tamara Navarro ${ }^{1}$, Richard Parrish ${ }^{1}$, Nav Persaud ${ }^{29}$, Kevin Pottie ${ }^{8}$, Gabriel Rada ${ }^{17,30}$, Ludovic Reveiz ${ }^{31}$, Anne Hayes ${ }^{32}$, Tanja Kuchenmüller ${ }^{33}$, Nicole Mittman ${ }^{34}$, Rachel Rodin ${ }^{7}$, Waleed Alhazzini ${ }^{1}$, Pablo Alonso-Coello ${ }^{35}$, Jan Brozek ${ }^{1}$, Lisa Hartling ${ }^{36}$, Mark Loeb ${ }^{1}$, Lawrence Mbuagbaw ${ }^{1}$, Joerg J. Meerpoh ${ }^{37}$, Reem A. Mustafa ${ }^{38}$, Robby Nieuwlaat ${ }^{1}$, Nancy Santesso ${ }^{1}$, Holger J. Schünemann ${ }^{1}$

Participating groups: Cochrane Canada, Cochrane Canada Francophone, Cochrane Child Health, Cochrane Chile, Cochrane Czech Republic, Cochrane Germany, Cochrane Effective Practice and Organisation of Care, Cochrane Hypertension, Cochrane Iberoamérica, Cochrane Norway, Cochrane South Africa, Campbell and Cochrane Equity Methods Group, Cochrane Consumer Network Executive, Campbell Collaboration, GRADE Working Group and centers, Czech Republic Centre for Evidence-Based Healthcare, Guidelines International Network, Postgraduate Institute of Medical Education and Research, India, World Health Organization Collaborating Centre for Infectious Diseases, Research Methods and Recommendations, Pan American Health Organization, World Health Organization

\section{Corresponding Author: Adrienne Stevens: adrienne.stevens@mcmaster.ca}

\section{Introduction and background}

The rapidly unfolding Coronavirus Disease of 2019 (COVID-19) pandemic and an ever-increasing volume of relevant literature presents considerable strain for decision makers. Those decision makers are interested in information not only about benefits and harms of interventions but also contextual information such as people's values and preferences, costs, equity, acceptability, and feasibility. The technical and time-consuming effort required to gather, assess, synthesize, and evaluate the certainty of relevant evidence using GRADE is not realistic for the individual clinician, public health official, policy, patient, caregiver, or citizen decision maker.
Our large, international collaborative team is building a platform that will present to all stakeholders a living, comprehensive map of recommendations from high-quality guidelines, along with their evidence base (Figure 1). A key feature of the platform is providing a gateway to allow users to decide whether to adopt the available recommendation as it is, adapt it to their context, or create a de novo recommendation, a process known as 'adolopment'.[1] To our knowledge, this is the first living map of recommendations on COVID-19 to include an interactive feature that supports users in 'adoloping' existing recommendations using the GRADE Evidenceto-Decision (EtD) Frameworks.[2-4] The platform also harnesses

\section{Joint first authors.}

${ }^{1}$ McMaster University, Canada; ${ }^{2}$ American University of Beirut, Lebanon; ${ }^{3}$ National Institute for Health Technology Assessment, Brazil; ${ }^{4}$ Hospital Moinhos de Vento, Brazil; ${ }^{5}$ South African Medical Research Council, South Africa; ${ }^{6}$ Postgraduate Institute of Medical Education and Research, India; ${ }^{7} \mathrm{Public}$ Health Agency of Canada, Canada; ${ }^{8}$ University of Ottawa, Canada; ${ }^{~ B}$ ruyère Research Institute, Canada; ${ }^{10}$ Evidence Prime, Poland; ${ }^{11}$ Norwegian Institute of Public Health, Norway; ${ }^{12}$ University of Oslo, Norway; ${ }^{13}$ Masaryk University, Czech Republic; ${ }^{14}$ Institute for Health Information and Statistics of the Czech Republic, Czech Republic; ${ }^{15} \mathrm{Czech}$ Health Research Council, Czech Republic; ${ }^{16}$ University of Amsterdam, Netherlands; ${ }^{17}$ World Health Organization, Switzerland; ${ }^{18}$ Pontificia Universidad Católica de Chile, Chile; ${ }^{19}$ Labrador-Grenfell Health, Canada; ${ }^{20}$ World Health Organization - Regional Office for Europe, Denmark; ${ }^{21}$ Cochrane Consumer Executive, Canada; ${ }^{22} \mathrm{Ottawa}$ Hospital Research Institute, Canada; ${ }^{23}$ Université Laval, Canada; ${ }^{24}$ University of British Columbia, Canada; ${ }^{25} \mathrm{Lazio}$ Regional Health Service, Italy; ${ }^{26}$ St Joseph's Healthcare Hamilton, Canada; ${ }^{27}$ Guidelines International Network, Austria; ${ }^{28}$ University of Adelaide, Australia; ${ }^{29}$ University of Toronto, Canada; ${ }^{30}$ Epistemonikos Foundation, Chile ; ${ }^{31}$ Pan American Health Organization, USA; ${ }^{32}$ Ontario Ministry of Health, Canada; ${ }^{33}$ World Health Organization, Switzerland; ${ }^{34}$ Canadian Agency for Drugs and Technologies in Health, Canada; ${ }^{35}$ Biomedical Research Institute Sant Pau (IIB Sant PauCIBERESP), Spain; ${ }^{36}$ University of Alberta, Canada; ${ }^{37}$ University of Freiburg, Germany; ${ }^{38}$ Kansas University Medical Centre, USA 


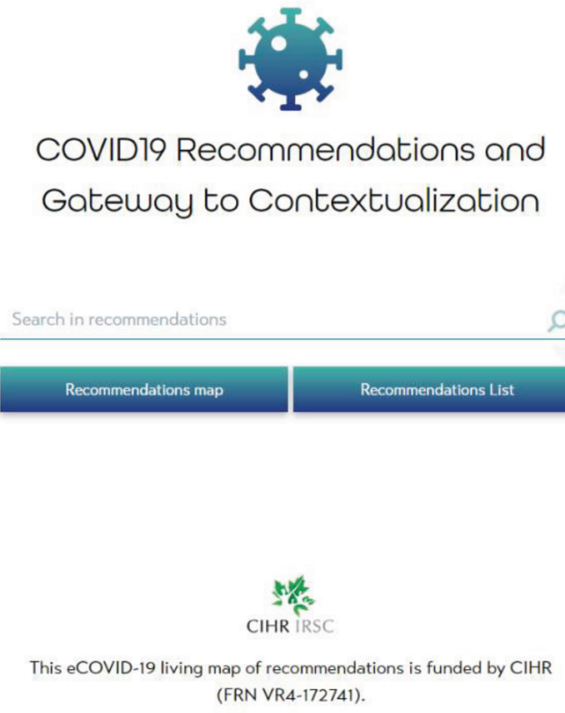

Figure 1. eCOVID19 Guidelines: the catalogue of recommendations

decisions made by users to then share with other users of the map, further adding novelty.

Our aim is to cover a broad array of COVID-19 topics. We intend for this map to serve as a knowledge translation vehicle to help to ease the burden on decision makers by providing access to information to facilitate evidence-informed decisions. By capitalising on efforts invested to date in COVID-19 guideline development, we will reduce waste.

Although our platform maps COVID-19 recommendations, we link to a separate portal, produced by the Norwegian government, that maps COVID-19 evidence (fhi.no/en/qk/systematic-reviews-hta/ map/). We will present our living recommendations map in both official languages of Canada, English and French, but with the hope of translation to other languages.

Led and centrally co-ordinated by Cochrane Canada and the World Health Organization (WHO) Collaborating Centre (WHO-CC) for Infectious Diseases, Research Methods and Recommendations at McMaster University, our team includes Cochrane-, GRADE-, $\mathrm{JBI}$ - and G-I-N-affiliated groups, key investigators situated in low-and-middle-income settings, Cochrane Consumer leadership, clinical and policy decision makers (including public health leaders directly connected with Canadian indigenous communities), artificial intelligence and information technology experts, software developers, and language translators. In addition to the key linkage with the Norwegian Institute of Public Health (NIPH)'s Systematic and Living Map on COVID-19 Evidence, the McMaster's COVID-19 Evidence Alerts (plus.mcmaster.ca/COVID-19), Pan American Health
Organization (PAHO)'s BIGG Database (sites.bvsalud.org/bigg/ biblio), and the Epistemonikos Living OVerview of Evidence (L•OVE) Platform (iloveevidence.com) resources are integral to acquiring the guideline and contextual literature and for portal linking. This map will also provide access to information to the WHO Model List of Essential Medicines.

Roles of participating individuals and groups collectively span informing the methodology, populating the recommendations map, disseminating the map, and facilitating adolopment with key stakeholder partners through professional networks.

\section{Key activities and strategies}

A prototype of the recommendations map was developed for the grant funding submission and is based on previous collaborative work between a subset of this group (MacGRADE and Evidence Prime) and the World Health Organization Global Tuberculosis Department (tuberculosis.evidenceprime.com).

We formed a project Executive Team that has met weekly since mid-July to guide the overall methodological and development approach, in concert with Evidence Prime's refinement and customisation of the electronic map and GRADEpro software (gradepro.org). A total of 10 working groups have been formed according to the various activities related to the totality of the work, such as literature searching, guideline appraisal, equity considerations, language translation, and activities related to facilitating the adolopment of recommendations by stakeholders. These groups report their activities and decisions back to the 
Executive Team. Research teams mobilised from within the grant team participate in training and calibration exercises prior to extracting guideline and recommendation information and constructing the EtD frameworks in GRADEpro. We will seek feedback from knowledge users within our grant team to refine its development. A consultancy team comprising clinical, public health, and consumer expertise will allow us to seek pertinent content and contextual feedback as we develop the map.

Modules housed within the portal facilitate user-directed adolopment, that is, they support the user in how to consider the evidence and additional considerations provided in the EtD domains to inform their judgement of what the recommendation should be for their context and any additional considerations for its application.

Current funding allows us to undertake this work as a living project through May 2021 with daily searches of the guideline literature and make it freely available globally. We are planning relevant methodological projects, such as assessing processes for improving the timeliness of guideline development and how to assess and mitigate inequities that may be magnified in the context of the pandemic.

\section{Outcomes and impact of activities}

We will publicly launch this platform when it is populated with a sufficient volume of EtDs and has undergone satisfactory user testing; we will make the link available through Cochrane Canada's social media channels. We would highly desire closer linkages with Cochrane and other groups to create synergies in the development and use of this map. In addition to disseminating the outcomes of the methodological projects, we plan to share examples of how this tool has impacted COVID-19 decision making.

\section{Lessons for the future: sustainability and transferability}

The expected volume of work and extensive co-ordination required to bring together and align the multiple working groups and internationally situated research sites necessitates the funding we were successful in receiving. However, additional funding would be needed to accommodate literature growth and continued decision support in the long term. We have been able to mobilise quickly on funding notice because of established collaborations, strong leadership and team cohesion, and development of the platform prototype prior to the receipt of funds. As the work unfolds, our experience and lessons learned will inform future efforts of building and using interactive electronic maps of evidence and recommendations that allow and harness user-directed adolopment.

\section{Declarations of interest}

Evidence Prime is a for-profit company co-owned by McMaster University and information technology experts. Depending on the type of use, licensing fees may apply for using GRADEpro GDT but not for the use of Evidence to Decision Frameworks produced in this study. JB, AN, BD and JL are employed by Evidence Prime (JB is employed outside of this submitted work). AN, BD and JL are shareholders of Evidence Prime Inc.

Grading of Recommendations, Development, and Evaluation (GRADE) Working Group and Centres members and staff include AS, TL, EAA, MF, TK, RBP, DKC, SD, SF, AHaj, AI, MK, MWL, IN, TP, PT, GV, VW, MD, LK, JK, ZM, GR, KS, PA, JB, JJM, RAM, RN, NS and HJS. The GRADE software is GRADEpro, which is programmed by Evidence Prime. Guidelines International Network (G-I-N) members and staff are TK, JLM, MK, MWL, EH, LK, JK, ZM, PAC, JJM and HJS.

The following Cochrane affiliations are acknowledged with author initials listed in parentheses: Cochrane Canada Centre (AS, SD, Al, KS, WA, JB, LB, RN, NS, HJS); Cochrane Canada Francophone (AT); Cochrane Child Health (LH); Cochrane Chile (IN, GR); Cochrane Cystic Fibrosis and Genetic Disorders (IO); Cochrane Czech Republic (MK, LK, JK); Cochrane Effective Practice and Organisation of Care (SF); Cochrane Germany (JJM); Cochrane Global Ageing (VW); Cochrane Governing Board (TK); Cochrane GRADEing (EAA, ML, JJM, GV, VW, SF; JB, NS, HJS); Cochrane Hypertension (JMW); Cochrane Musckuloskeletal, Oral, Skin, and Sensory Network (PT); Cochrane Norway (SF); Cochrane Priority Setting Methods (VW); Cochrane Prognosis Methods (IO); Cochrane Rapid Reviews Methods (AS); Cochrane South Africa (TK); Campbell and Cochrane Equity Methods Group (OD, PT, VW, KP); Cochrane Consumer Executive (MS); Cochrane Iberoamérica (PAC).

LR is a staff member of the Pan American Health Organization and LM, MR and TKuc are staff members of the World Health Organization. The authors alone are responsible for the views expressed in this publication. They do not necessarily represent the decisions or policies of funding or employing organisations.

Any study team member who is a co-author on prospective guidelines (or underlying evidence synthesis products) included in the platform would not be involved in its appraisal or extraction or in any quality control processes implemented in the development of the platform. In addition, any clinical or contextual advisors with conflicts of interest relevant to the included guidelines would abstain from providing clinical feedback.

\section{Funding}

This study is funded by the Canadian Institutes of Health Research, funding reference number VR4-172741. The funder is not involved in the development of the protocol, undertaking of the research, 
dissemination of relevant products, nor decision to publish this work in any form. HS also reports grants from the World Health Organization during the conduct of this study.

\section{Additional resources}

For information on guideline development, refer to g-i-n.net.

To access GRADEpro software, consult gradepro.org.

\section{Acknowledgements}

We are grateful to the many researchers and trainees who will be taking part in developing the platform from across the participating research sites and to personnel from collaborating organisations who are helping to make this work possible.

\section{References}

1. Schünemann HJ, Wiercioch W, Brozek J, Etxeandia-Ikobaltzeta I, Mustafa RA, Manja V, et al. GRADE Evidence to Decision (EtD) frameworks for adoption, adaptation, and de novo development of trustworthy recommendations: GRADE-ADOLOPMENT. Journal of Clinical Epidemiology 2017;81:101-10. https://doi:10.1016/j. jclinepi.2016.09.009

2. Alonso-Coello P, Schünemann HJ, Moberg J, Brignardello-Petersen R, Akl EA, Davoli M, et al. GRADE Evidence to Decision (EtD) frameworks: a systematic and transparent approach to making well informed healthcare choices. 1: Introduction. BMJ 2016;353:i2016. https://doi. org/10.1136/bmj.i2016

3. Schünemann HJ, Mustafa R, Brozek J, Santesso N, Alonso-Coello P, Guyatt G, et al. GRADE Guidelines: 16. GRADE evidence to decision frameworks for tests in clinical practice and public health. Journal of Clinical Epidemiology 2016;76:89-98. https://doi.org/10.1016/j. jclinepi.2016.01.032

4. Moberg J, Oxman AD, Rosenbaum S, Schünemann HJ, Guyatt G, Flottorp S, et al. The GRADE Evidence to Decision (EtD) framework for health system and public health decisions. Health Research Policy and Systems 2018;16(1):45. https://doi.org/10.1186/s12961-018-0320-2 


\title{
International collaborations with Cochrane Oral Health to inform the re-opening of dental practices
}

\author{
Authors: Jan E Clarkson ${ }^{1,2}$, Anne-Marie Glenny ${ }^{2}$, Helen V Worthington ${ }^{2}$, Craig R Ramsay ${ }^{3,4}$ \\ Participating groups: Cochrane Oral Health, Cochrane ENT, SDCEP: Scottish Dental Clinical Effectiveness \\ Programme, COVID-19 Dental Services Evidence Review (CoDER) Working Group, NHS Education for Scotland, \\ University of Aberdeen, University of Dundee, The University of Manchester, World Health Organization, FDI World \\ Dental Federation, Global Evidence Ecosystem for Oral Health (GEEOH)
}

Corresponding Author: Helen Worthington: helen.worthington@manchester.ac.uk

\section{Introduction and background}

The COVID-19 pandemic resulted in the closure of dental services across the world due to the use of aerosol-generating procedures (AGPs) and the close contact between dental professionals and patients. During this initial period, for the safety of both patients and dental professionals, only emergency treatment was provided, which consisted of advice, analgesics, antibiotics, and just the extraction of teeth. This left many people in pain with acute infections, reminiscent of dentistry in medieval times. The lifting of the initial lockdown imposed by most countries, due to COVID-19, has left uncertainty about how to safely reopen dental practices in order to return to providing routine dental care for patients.

\section{Key activities and strategies}

The participating groups (see full list under acknowledgements) worked on multiple projects that assisted in providing policy makers with information for making decisions about re-opening dental practices.

Cochrane Oral Health launched a webpage that collated COVID19-relevant guidance documents for dental practice (oralhealth. cochrane.org/news/covid-19-coronavirus-resources-oral-anddental-care-team) and also linked to relevant systematic reviews and other documents. We followed the structure of the Cochrane ENT COVID-19 website (ent.cochrane.org/news/covid-19coronavirus-disease-ent-hearing-balance).

Cochrane Oral Health has a leading role in the COVID-19 Dental Services Evidence Review (CODER) Working Group, led by Jan Clarkson, joint Co-ordinating Editor of Cochrane Oral Health, and
Craig Ramsay. This is a key collaboration that reviews available evidence for dental services to support policy decisions. Their first report highlights specific international guidance for dentists reopening their practices (or not), which was based on 16 countries. [1] This rapid review collates and summarizes recommendations from the various sources identified within five themes relevant to the re-opening of dental services: practice preparation; personal protective equipment, management of the clinical area; dental procedures; and cleaning and disinfection. This document included guidance for treating patients with and without COVID-19. The second rapid review undertaken by the CoDER Working Group looked at the specific use of AGPs for patients with and without COVID-19, from 63 guidance documents from 58 countries, and was also posted on the Cochrane Oral Health website.[2]

Common issues of importance to both Cochrane Oral Health and Cochrane ENT over the use of antimicrobial mouthwashes initiated the joint undertaking of three Cochrane rapid systematic reviews. [3-5] We also prioritized a Cochrane COVID-19 rapid review on interventions to reduce contaminated aerosols produced during dental procedures.[6]

\section{Outcomes and impact of activities}

A global audience accessed the CoDER guidance for re-opening dental services from 16 countries 45,000 times. [1] This interest was probably helped by the report being featured on the main Cochrane website. This work was built on collaboration with the Global Evidence Ecosystem for Oral Health (GEEOH), the World Health Organization (WHO), and FDI World Dental Federation. The WHO referenced the report in a briefing document, [7] and this led to the FDI World Dental Federation extending the review to include

${ }^{1}$ University of Dundee, UK; ${ }^{2}$ Cochrane Oral Health, University of Manchester, UK; ${ }^{3}$ University of Aberdeen, UK; ${ }^{4}$ Cochrane Effective Practice and Organisation of Care, UK 
new guidance documents from other countries as they become available. In the report, there is a highly variable level of detail given across the international sources. Within these international sources, for most of the statements included there was no reference underpinning the evidence, and some of them are unlikely to have strong (or any) research evidence.

The second rapid review from the CoDER Working Group on the use of AGPs was viewed 8000 times.[2] The UK documents provided similar guidance, with duplication of effort, which could be avoided with more collaboration and sharing. [8-11]

The three rapid reviews on the use of antibacterial mouthwashes have been published jointly on the Cochrane Library by Cochrane Oral Health and Cochrane ENT,[3-5] as well as a rapid review on interventions to reduce contaminated aerosols.[6]

A further rapid review developed by Scottish Dental Clinical Effectiveness Programme with the support of Cochrane Oral Health has been undertaken of the evidence related to the mitigation of AGPs in dentistry and the associated risk of transmission of COVID-19. We set up a multidisciplinary working group comprising subject specialists from disciplines including particle physics, aerobiology, and clinical virology, in addition to those performing multiple roles within dentistry. Representatives from all four of the devolved UK nations participated in the work of the Group, including the Chief Dental Officers. The review is published on the Scottish Dental Clinical Effectiveness Programme website and linked from the Cochrane Oral Health website.[12]

\section{Lessons for the future: sustainability and transferability}

There was a major benefit in using all the links we already had to come together as the CODER Working Group and to obtain information as rapidly as possible. For example, Working Group initially had data from 16 countries for the first set of guidance, and this increased to 63 countries in the second rapid review, using our new contacts. Cochrane helped in increasing the exposure to the first rapid review through promoting and engaging with the community. For future preparedness it is important to build on utilizing Cochrane's processes and platforms, particularly for evidence relevant to urgent policy decision making.

Our response to COVID-19 has assessed global evidence to help inform decisions; however, to date as far as we know only the UK has used the guidance to inform decisions. In future we would be more proactive about bringing people together, enabling conversations about the global strategy that could benefit more counties globally. We will invite, through the Global Evidence Ecosystem for Oral Health (GEEOH), international organizations along with $\mathrm{WHO}$ and its network of international chief dental officers. We would enable a rapid search and summaries of the evidence to discuss and make available to facilitate collaboration to avoid duplication of effort. A positive outcome of our recent collaboration with key stakeholders is a respect and willingness to work together differently and to move rapidly and collectively in the event of a future pandemic.

\section{Acknowledgements}

We would like to thank and acknowledge the work done by the COVID-19 Dental Services Evidence Review (CoDER) Working Group: Magaly Aceves-Martins (University of Aberdeen, UK); Miriam Brazzelli (University of Aberdeen, UK); Gareth Calvert (NHS Greater Glasgow and Clyde, UK); Thibault Colloc (NHS Grampian, UK); Manas Dave (University of Manchester, UK); Beatriz Goulão (University of Aberdeen, UK); Thomas J Lamont (University of Dundee, UK); Derek Richards (University of Dundee, UK); Philip Riley (Cochrane Oral Health, University of Manchester, UK); Clare Robertson (University of Aberdeen, UK); Tanya Walsh (Cochrane Oral Health, University of Manchester, UK); and Gavin J Wilson (University of Leeds, UK).

We would like to thank and acknowledge the contribution of the following individuals for providing the advice and access to the international guidance documents necessary for this rapid review: Colette Bridgman (Chief Dental Officer, Wales); Alonso Carrasco-Labra (ADA Science \& Research Institute); Riana Clarke (National Clinical Director Oral Health, New Zealand); Michael Donaldson (Chief Dental Officer, Northern Ireland); Tom Ferris (Chief Dental Officer, Scotland); Sara Hurley (Chief Dental Officer, England); Paulo Melo (COVID-19 Task Team Chair, FDI World Dental Federation); Timothy Ricks (Chief Dental Officer, US Public Health Service); James Taylor (Chief Dental Officer, Canada); and Benoit Varenne (Dental Officer, World Health Organization). Thanks also to Tif Qureshi for providing access to some international guidance documents.

We are also grateful for the help and support provided by Shona Floate (University of Glasgow), Anne Littlewood (Cochrane Oral Health), Laura MacDonald (Cochrane Oral Health), David Felix (Postgraduate Dental Dean, NHS Education for Scotland), and colleagues from NHS Education for Scotland's Clinical Effectiveness workstream: Samantha Rutherford, Douglas Stirling, Michele West, and Linda Young.

\section{Declarations of interest}

$\mathrm{JC}$ and $\mathrm{AG}$ are Co-ordinating Editors and HW is an Editor with Cochrane Oral Health. CR is Statistical Editor with Cochrane Effective Practice and Organisation of Care. The authors declare that they have no other competing interests.

\section{Funding}

We obtained no direct funding for these projects. The authors receive ongoing funding from: the School of Dentistry, The University of Manchester, UK; the University of Aberdeen, UK; the University of Dundee, UK; the Chief Scientist Office, Scotland, UK; and the UK National Institute for Health Research.

\section{References}

1. COVID-19 Dental Services Evidence Review (CoDER) Working Group. Recommendations for the re-opening of dental services: a rapid 
review of international sources. Version 1.3; May 2020. Available from oralhealth.cochrane.org/news/recommendations-re-opening-dentalservices-rapid-review-international-sources

2. Clarkson J, Ramsay C, Richards D, Robertson C, Aceves-Martins M, on behalf of the CoDER Working Group. Aerosol generating procedures and their mitigation in international dental guidance documents - a rapid review. Available from oralhealth.cochrane.org/news/aerosolgenerating-procedures-and-their-mitigation-international-guidancedocuments (accessed on 28 September 2020)

3. Burton MJ, Clarkson JE, Goulao B, Glenny A-M, McBain AJ, Schilder AG, et al. Use of antimicrobial mouthwashes (gargling) and nasal sprays by healthcare workers to protect them when treating patients with suspected or confirmed COVID-19 infection. Cochrane Database of Systematic Reviews 2020;(9):CD013626. https://doi. org/10.1002/14651858.CD013626.pub2

4. Burton MJ, Clarkson JE, Goulao B, Glenny A-M, McBain AJ, Schilder $A G$, et al. Antimicrobial mouthwashes (gargling) and nasal sprays administered to patients with suspected or confirmed COVID-19 infection to improve patient outcomes and to protect healthcare workers treating them. Cochrane Database of Systematic Reviews 2020;(9):CD013627. https://doi.org/10.1002/14651858.CD013627.pub2

5. Burton MJ, Clarkson JE, Goulao B, Glenny A-M, McBain AJ, Schilder $A G$, et al. Antimicrobial mouthwashes (gargling) and nasal sprays to protect healthcare workers when undertaking aerosol-generating procedures (AGPs) on patients without suspected or confirmed COVID-19 infection. Cochrane Database of Systematic Reviews 2020;(9):CD013628. https://doi.org/10.1002/14651858.CD013628.pub2

6. Kumbargere Nagraj S, Eachempati P, Paisi M, Nasser M, Sivaramakrishnan G, Verbeek JH . Interventions to reduce contaminated aerosols produced during dental procedures for preventing infectious diseases. Cochrane Database of Systematic Reviews 2020;(10):CD013686. https://doi.org/10.1002/14651858.CD013686.pub2

7. World Health Organization. Considerations for the provision of essential services in the context of COVID-19: interim guidance. 3 August 2020. https://apps.who.int/iris/rest/bitstreams/1289841/retrieve

8. Office of Chief Dental Officer, England. Standard operating procedure. Transition to recovery: a phased transition for dental practices towards the resumption of the full range of dental provision. Version 3, August 2020. Available from england.nhs.uk/coronavirus/primary-care/dentalpractice

9. Health and Social Care Board, Northern Ireland. Preparation for the re-establishment of the General Dental Services: operational guidance. Updated 22 June 2020. hscbusiness.hscni.net/pdf/Preparation for the Re-establishment of the GDS Updated 22 June 2020.pdf

10. Scottish Dental Clinical Effectiveness Programme. Resuming general dental services following COVID-19 shutdown: a guide and implementation tools for general dental practice. For Phases 2 and 3 of dental services remobilisation. Version 1.1, June 2020. Available at www.sdcep.org.uk/published-guidance/covid-19-practice-recovery

11. All Wales Clinical Dental Leads COVID-19 Group. Standard operating process for non-COVID-19 dental centres providing aerosol generating procedures in Wales. June 2002. Available at gov.wales/providingaerosol-generating-procedures-agp-non-covid-19-dental-patientsguidance

12. Scottish Dental Clinical Effectiveness Programme. Mitigation of aerosol generating procedures in dentistry: a rapid review. Version 1.0, September 2020. Available from sdcep.org.uk/published-guidance/ covid-19-practice-recovery/rapid-review-of-agps 


\title{
Cochrane ENT in the COVID-19 pandemic: using our expertise and collaborating effectively
}

\author{
Authors: Bellorini $\mathrm{J}^{1}$, Cox $\mathrm{S}^{1}$, Webster $\mathrm{KE}^{1}$, Burton $\mathrm{MJ}^{1}$ \\ Participating groups: Cochrane ENT, Cochrane Oral Health, Cochrane Musculoskeletal, Oral, Skin and Sensory \\ Corresponding Author: Jenny Bellorini: jenny.bellorini@nds.ox.ac.uk
}

\section{Introduction and background}

The 2020 COVID-19 pandemic has presented enormous challenges for healthcare systems globally. At Cochrane ENT (ent.cochrane. org) we wanted to see how we could use our particular skills and resources to assist professionals and patients in our own clinical field.

In the early months of the pandemic, clinicians were presented with a blizzard of information in the form of resources, guidance and advice statements from international organizations and societies. These related to COVID-19 and its impact on the management of patients with ENT, hearing and balance problems by ENT and audiology professionals. In Cochrane ENT we felt that we could use our information management expertise to search systematically for these resources, and to collate and structure them in an online repository that would be useful to our professional colleagues. Hopefully, more useful than a simple list of documents and hyperlinks that had randomly been identified or sent to us.

It also quickly became clear that healthcare workers at the forefront of the COVID-19 pandemic are at risk of infection due to repeated exposure to infected, or potentially infected, patients. Although the risk of infection may be reduced by the use of personal protective equipment (PPE), healthcare workers may be especially at risk when undertaking 'aerosol-generating procedures' (AGPs), which are common in ENT and dental practice. We identified an interest in our communities in looking at interventions to add to the use of PPE when doing AGPs.

Finally, members of Cochrane ENT identified early in the pandemic that olfactory dysfunction/anosmia (loss of sense of smell) was a cardinal and sometimes persistent symptom of COVID-19 infection.

\section{Key activities and strategies}

In April 2020, we used the expertise of our Information Specialist to begin to develop a curated repository of guidance and evidence relevant to the management of patients with ENT, hearing, and balance problems during the COVID-19 pandemic (ent.cochrane. org/news/covid-19-coronavirus-disease-ent-hearing-balance). As further evidence has become available, we have expanded and updated this resource, and we will continue to maintain it.

As anecdotal evidence began to emerge that healthcare workers were using antimicrobial mouthwashes and nasal sprays to protect themselves and their patients against COVID-19 infection, in collaboration with Cochrane Oral Health (oralhealth.cochrane. org) we prioritized, registered and fast-tracked three systematic reviews of these interventions for the prevention of COVID-19 infection.

As the pandemic has continued it has become clear that olfactory dysfunction is a common and, in some patients, persistent symptom of COVID-19 infection, with the potential to cause significant reduction in quality of life.[1] In response to a call from the UK National Institute for Health Research for research on 'COVID-19: Recovery and Learning', Cochrane ENT applied for funding to complete a set of Cochrane living systematic reviews on the prevention and treatment of post-COVID-19 anosmia. Our bid was successful and work on the fast-tracked reviews began in September 2020.

\section{Outcomes and impact of activities}

Our maintained online repository of international clinical guidance on patient care during the pandemic is a useful resource for our professional colleagues in ENT and audio-vestibular care. 
The three fast-tracked systematic reviews of antimicrobial mouthwashes and nasal sprays to protect healthcare workers and patients from COVID-19 infection, produced jointly with Cochrane Oral Health, were published in September 2020.[2-4] While we cannot yet ascertain the benefits and harms of these agents, we have identified a number of ongoing studies and we will update the reviews as the results become available. We have some concerns, from the limited information currently available, that adverse effects are not being thoroughly assessed in the current ongoing studies. We have found no planned studies addressing the important question of the use of these agents during AGPs (e.g. nasal endoscopy or routine dental treatment such as drilling/scaling). However, this is a very fast-moving area. We hope that our early comments on adverse effect reporting in the ongoing studies that we have seen will be noted and acted upon by those planning future studies.

We are working to a target date of December 2020 for completion of the first iteration of our living systematic reviews on the prevention and treatment of post-COVID-19 anosmia. Currently we are working with two patient groups on the development of a core set of outcome measures for use in the reviews and work on the protocols is underway.

\section{Lessons for the future: sustainability and transferability}

Responding nimbly to the challenge of a global healthcare crisis of direct relevance to our clinical field was a steep learning curve. However, we learned that we do have specific skills, resources and contacts within Cochrane ENT that are useful and can be deployed, and that with flexibility we can divert resources away from other work to achieve this. Working in close collaboration with Cochrane Oral Health on the three jointly produced Cochrane reviews was a genuine pleasure and an experience that we would be keen to repeat with other groups. As most of the senior editorial staff from the two Cochrane Review Groups co-authored these reviews, this work was greatly facilitated by the editorial support of the Senior Editor and Associate Editor of the Cochrane Musculoskeletal, Oral, Skin and Sensory network. We believe that this is a good example of the potential for collaborative working within a Cochrane Network. This collaboration was also facilitated by the Cochrane Central Executive Team, who developed the functionality to allow the first full 'joint' publication in the Cochrane Library of reviews by multiple Cochrane groups.

\section{Additional resources}

Cochrane ENT repository of guidance: COVID-19 (coronavirus disease) - ENT, Hearing \& Balance: ent.cochrane.org/news/covid-19-coronavirusdisease-ent-hearing-balance

Fast-tracked reviews of antimicrobial mouthwashes (gargling) and nasal sprays:

- Use of antimicrobial mouthwashes (gargling) and nasal sprays by healthcare workers to protect them when treating patients with suspected or confirmed COVID-19 infection cochranelibrary.com/ cdsr/doi/10.1002/14651858.CD013626.pub2/full

- Antimicrobial mouthwashes (gargling) and nasal sprays administered to patients with suspected or confirmed COVID-19 infection to improve patient outcomes and to protect healthcare workers treating them: cochranelibrary.com/cdsr/ doi/10.1002/14651858.CD013627.pub2/full

- Antimicrobial mouthwashes (gargling) and nasal sprays to protect healthcare workers when undertaking aerosolgenerating procedures (AGPs) on patients without suspected or confirmed COVID-19 infection: cochranelibrary.com/cdsr/ doi/10.1002/14651858.CD013628.pub2/full

\section{Acknowledgements}

This project was supported by the UK National Institute for Health Research, via Cochrane Infrastructure, Cochrane Programme Grant, or Cochrane Incentive funding to Cochrane ENT. The views and opinions expressed therein are those of the authors and do not necessarily reflect those of the Systematic Reviews Programme, NIHR, NHS or the Department of Health.

\section{Declarations of interest}

JB is the Managing Editor for Cochrane ENT. SC is the Information Specialist for Cochrane ENT. KW is a Systematic Reviewer for Cochrane ENT. MB is the Co-ordinating Editor of Cochrane ENT. The authors declare no additional interests.

\section{Funding}

Cochrane ENT receives infrastructure funding from the UK National Institute for Health Research.

\section{References}

1. Hopkins C, Surda P, Whitehead E, Kumar BN. Early recovery following new onset anosmia during the COVID-19 pandemic - an observational cohort study. Journal of Otolaryngology - Head \& Neck Surgery 2020;49(1):26. https://doi.org/10.1186/s40463-020-00423-8

2. Burton MJ, Clarkson JE, Goulao B, Glenny A-M, McBain AJ, Schilder AG, et al. Use of antimicrobial mouthwashes (gargling) and nasal sprays by healthcare workers to protect them when treating patients with suspected or confirmed COVID-19 infection. Cochrane Database of Systematic Reviews 2020;(9):CD013626. https://doi. org/10.1002/14651858.CD013626.pub2

3. Burton MJ, Clarkson JE, Goulao B, Glenny A-M, McBain AJ, Schilder $A G$, et al. Antimicrobial mouthwashes (gargling) and nasal sprays administered to patients with suspected or confirmed COVID-19 infection to improve patient outcomes and to protect healthcare workers treating them. Cochrane Database of Systematic Reviews 2020;(9):CD013627. https://doi.org/10.1002/14651858.CD013627. pub2

4. Burton MJ, Clarkson JE, Goulao B, Glenny A-M, McBain AJ, Schilder AG, et al. Antimicrobial mouthwashes (gargling) and nasal sprays to protect healthcare workers when undertaking aerosol-generating procedures (AGPs) on patients without suspected or confirmed COVID-19 infection. Cochrane Database of Systematic Reviews 2020;(9):CD013628. https://doi.org/10.1002/14651858.CD013628.pub2 


\title{
Cochrane Childhood Cancer and COVID-19: developing recommendations for childhood, adolescent and young adult cancer survivors
}

\author{
Author: Elvira C van Dalen ${ }^{1,2,3}$ \\ Participating groups: Cochrane Childhood Cancer, International Late Effects of Childhood Cancer Guideline \\ Harmonization Group
}

Corresponding Author: Elvira C van Dalen: E.C.vanDalen@prinsesmaximacentrum.nl

\section{Introduction and background}

Over the last decades, childhood, adolescent, and young adult (CAYA) cancer survival has improved dramatically. It currently exceeds $80 \%$ in children diagnosed with cancer in developed countries.[1] Unfortunately many survivors will experience so called late adverse events later in life. Approximately $75 \%$ will have at least one adverse event and almost $25 \%$ will have as many as five or more adverse events.[2] These adverse events are very diverse, including secondary neoplasms and different types of organ dysfunction (e.g. cardiac, pulmonary, endocrine).[2] In addition, survivors may also be at risk of premature physiological aging.[3]

Shortly after the World Health Organization (WHO) declared COVID-19 a pandemic, healthcare practitioners involved in the care of CAYA cancer survivors started to receive questions from worried survivors. Emerging evidence from the general population showed a possible increased risk of a severe course of disease in older people and in people with comorbidities like cardiac disease and diabetes. [4] These are precisely the problems many CAYA cancer survivors face and they may thus be at risk of a severe course of COVID-19. However, at the time little was known about COVID-19 and CAYA cancer survivors.

To help answer the questions, members of Cochrane Childhood Cancer (childhoodcancer.cochrane.org) and the International Late Effects of Childhood Cancer Guideline Harmonization Group (IGHG; www.ighg.org) joined forces.

\section{Key activities and strategies}

The IGHG co-chairs, Leontien Kremer (also a Co-ordinating Editor at Cochrane Childhood Cancer) and Melissa Hudson, assembled an international COVID-19 working group. It consists of paediatric oncologists, late effects clinicians, supportive care specialists, infectious disease specialists, psychologists, patient representatives, and survivorship researchers.

The objectives were to summarize the existing evidence and worldwide recommendations on conditions associated with a severe course of COVID-19, both in CAYA cancer survivors and other populations, and to develop a consensus statement to provide guidance for healthcare practitioners and CAYA cancer survivors regarding COVID-19. The working group defined a severe course of COVID-19 as hospitalization, admission to the intensive care unit, use of mechanical ventilation or death, or a combination of these.

For the first objective we performed a systematic literature search (December 2019 to April 2020) including different sources. We summarized the evidence and formulated levels and conclusions of evidence. We also collected information on conditions associated with a high risk of a severe course of COVID-19 in the general population from the websites of the WHO and different national health institutions, which we consulted weekly between March and May 2020.

During working group discussions, we formulated recommendations for the following questions.

- Which CAYA cancer survivors are at higher risk of a severe course of disease?

- What measures should survivors take to reduce infection risk?

- What additional measures should survivors at high risk take?

- What should be done by a survivor who is at high risk and who develops COVID-19 symptoms?

- What are other effects of the COVID-19 pandemic?

${ }^{1}$ Princess Máxima Center for Pediatric Oncology, Utrecht, The Netherlands; ${ }^{2}$ Cochrane Childhood Cancer, The Netherlands; ${ }^{3}$ The International Late Effects of Childhood Cancer Guideline Harmonization Group 
As no information in CAYA cancer survivors was available, we extrapolated data from other populations to CAYA cancer survivors. When new information became available, we modified the recommendations accordingly.

More detailed information on the methodology of this project has been published.[5]

\section{Outcomes and impact of activities}

We were able to provide harmonized COVID-19 recommendations for CAYA cancer survivors within a relatively short period of time, through an international collaborative approach that balanced the scarcity of available information with the rapidly emerging need for guidance.

We have received many enthusiastic responses stating that our recommendations have been very helpful in these uncertain circumstances.

The recommendations are currently available in 14 languages (English, Dutch, German, French, Italian, Czech, Greek, Polish, Portuguese, Spanish, Croatian, Turkish, Chinese and Japanese). To reach as many people as possible they are disseminated through the Cochrane Childhood Cancer and IGHG websites, and different national and institutional paediatric cancer forums, such as the American Society of Pediatric Hematology/Oncology, the Japanese Society of Pediatric Hematology/Oncology, the Pan-European Network for Care of Survivors after Childhood and Adolescent Cancer, the Childhood Cancer International-Europe organization, and the European branch of the International Society of Pediatric Oncology.

By 5 September 2020 the IGHG website had been viewed 9486 times since the first version was published on 7 April 2020. Since then we have published two updates.

A manuscript describing the exact methodology of this project has been published.[5]

\section{Lessons for the future: sustainability and transferability}

The most important challenge we experienced was the scarcity of data, both in CAYA cancer survivors and the general population. This was something that was not unexpected under these circumstances and depending on the type of aetiological agent, either new or already known, we might encounter this again with future pandemics. However, the methodology developed during this COVID-19 project can effectively deal with this issue. For future pandemics this might lead to an even quicker response as the methodology is now already available.

The fact that there was already a collaborative platform with a large support base and extensive content and methodological knowledge from within both Cochrane Childhood Cancer and the IGHG, and the recognition by its members of the urgent need to summarize available evidence, has helped tremendously in preparing the recommendations in a timely manner. It also made it possible to disseminate the recommendations widely and quickly. It was encouraging to learn that if we face a similar challenge in the future, we have the ability to respond quickly again.

\section{Declarations of interest}

Elvira C van Dalen is the Co-ordinating Editor of Cochrane Childhood Cancer. She declares no other competing interests.

\section{Funding}

The Editorial Base of Cochrane Childhood Cancer is located in the Princess Máxima Center for Pediatric Oncology, Utrecht, the Netherlands.

\section{References}

1. Siegel RL, Miller KD, Jemal A. Cancer statistics, 2019. CA: A Cancer Journal for Clinicians 2019;69(1):7-34. https://doi.org/10.3322/ caac. 21551

2. Geenen MM, Cardous-Ubbink MC, Kremer LC, Van den Bos C, Van der Pal HJ, Heinen RC, et al. Medical assessment of adverse health outcomes in long-term survivors of childhood cancer. JAMA 2007;297(24):2705-15. https://doi.org/10.1001/jama.297.24.2705

3. Armenian SH, Gibson CJ, Rockne RC, Ness KK. Premature aging in young cancer survivors. JNCl: Journal of the National Cancer Institute 2019;111(3):226-32. https://doi.org/10.1093/jnci/djy229

4. Zhang J, Wang X, Jia X, Li J, Hu K, Chen G, et al. Risk factors for disease severity, unimprovement, and mortality in COVID-19 patients in Wuhan, China. Clinical Microbiology and Infection 2020;26(6):767-72. https://doi.org/10.1016/j.cmi.2020.04.012

5. Verbruggen LC, Wang Y, Armenian SH, Ehrhardt MJ, Van der Pal HJ, Van Dalen EC, et al. Guidance regarding COVID-19 for survivors of childhood, adolescent and young adult cancer: a statement from the International Late Effects of Childhood Cancer Guideline Harmonization Group. Pediatric Blood \& Cancer 2020;67:e28702. https://doi. org/10.1002/pbc.28702 


\title{
COVID-19 SHORT REPORT
}

\section{Collaborating to mobilize, update, and promote Cochrane evidence to support intensive care in the COVID-19 pandemic}

\author{
Authors: Teo Quay ${ }^{1,2}$, Harald Herkner ${ }^{2,3}$, Andrew Smith ${ }^{1,4}$ \\ Participating groups: Cochrane Emergency and Critical Care, Cochrane Anaesthesia \\ Corresponding Author: Harald Herkner: harald.herkner@mediuniwien.ac.at
}

\section{Introduction and background}

The COVID-19 pandemic thrust intensive care into the consciousness of the public and politicians in an unprecedented manner. As concern about the pandemic spread in early 2020, several opportunities arose for our two groups to contribute using a collaborative approach. Cochrane Emergency and Critical Care and Cochrane Anaesthesia have a long-shared history and up until 2018 were a single Review Group (Cochrane Anaesthesia, Critical and Emergency Care).[1] With this foundation, we were able to capitalize on our continued collaborative structure, shared editorial resources, and established relationships to ensure both groups could contribute optimally to Cochrane's COVID-19 response.

In the face of the lack of evidence specific to the virus, many existing reviews focusing on emergency and critical care, and airway and anaesthetic management would provide essential information to support decisions about the management of emergency and critical care for affected patients. Given the clinical focus areas and expertise of our two groups, we also realized our potential role in influencing COVID-19 secondary research efforts into the future.

\section{Key activities and strategies}

Our groups executed a multi-faceted approach. One initiative involved the promotion of existing reviews through contributions to the development of a Cochrane Library COVID-19 Special Collection on evidence relevant to critical care (in collaboration with the Acute and Emergency Care Network and the Editorial and Methods Department).[2] We also produced a separate Special Collection on the use of regional anaesthesia,[3] reflecting the risks of viral aerosol production associated with general anaesthesia and interest on avoiding shortages of sedative drugs. Cochrane Emergency and Critical Care was also involved in the scoping and prioritization process for COVID-19 reviews, prioritization of several rapid review updates, and registration of new rapid reviews, [4] and full systematic reviews relevant to the pandemic. Details about these ongoing initiatives are provided on the Cochrane Emergency and Critical Care website.[5]

The needs of the COVID-19 pandemic shaped the priority setting processes for both groups, which were underway at the time of the pandemic. As our two groups share an editorial office (a Managing Editor and Information Specialist), we re-distributed our editorial resources to ensure enhanced author support and expedited editorial processes, where feasible.

\section{Outcomes and impact of activities}

Overall, we aimed to ensure that our evidence was visible and accessible to as many end-users as possible. We realized our potential to be agile and flexible with established editorial processes and explored new collaborative opportunities. Based on the high utilization of the two Special Collections, $[2,3]$ the effort to consolidate and centralize evidence resulted in a helpful one-stopshop for users seeking evidence to inform future research or clinical practice.

Through consideration of the evolving evidence needs of the pandemic, Cochrane Emergency and Critical Care adjusted priority-setting procedures to ensure responsiveness to evolving healthcare challenges. This included pausing global priority setting to shift focus to COVID-19-relevant topics. We are also looking ahead, registering prospective meta-analyses and living systematic reviews to ensure that we stay current on topics with rapidly evolving evidence. The experience of this bootstrapped approach demonstrated that while adapting to public health crises and shifting evidence needs is challenging, it is achievable with the willingness of keen contributors and nimble editorial processes.

${ }^{1}$ Cochrane Anaesthesia, Denmark; ${ }^{2}$ Cochrane Emergency and Critical Care, Denmark; ${ }^{3}$ Medical University of Vienna, Austria; ${ }^{4}$ Royal Lancaster Infirmary, UK 
Another area of focus during these times is enhanced accessibility and visibility. Cochrane Anaesthesia is fortunate to have two Clinical Dissemination Fellows on the team. They are the first of their kind in Cochrane.[6] Their activities with the group have involved publishing summaries of reviews in professional newsletters, attending meetings to broker reviews to authors of national guidelines, promoting reviews on social media, and liaising with professional associations (e.g. the Obstetric Anaesthetists' Association) during health crises including drug supply shortages. [7] Our Dissemination Fellows spearheaded the Special Collection on regional anaesthesia, which had a substantial impact. Working with them is incredibly helpful as they understand the clinical significance of Cochrane evidence and how best to present it to fellow clinicians. Having frontline access and impact has been invaluable during the pandemic.

\section{Lessons for the future: sustainability and transferability}

The direct impact of the pandemic on many of the expert contributors we rely on as authors and editors was a big challenge. Many of these individuals were affected as they were called to the front line in clinical roles, assigned to priority research initiatives in their local contexts, or directly impacted on a personal level by the virus. Despite this strain, many contributors were willing to go above and beyond to help. Supporting them required transparent editorial processes and clear expectations, and contingency planning to ensure minimal disruption should their availability change over time. However, we have not had universal success. As a result of the above challenges, timelines have been delayed and projects abandoned. Our groups will continue to emphasize the importance of balancing strategic resource use and responsiveness while prioritizing the needs of stakeholders and rapidly changing contexts. Our experience provides a good template for how we might respond in the face of future health crises, and how we might strengthen our relationships with clinical and policy stakeholders to maximize the utility and impact of our reviews.

\section{Additional resources}

Cochrane Library Special Collection: Coronavirus (COVID-19): evidence relevant to critical care: cochranelibrary.com/collections/doi/SC000039/ full

Cochrane Library Special Collection: Coronavirus (COVID-19): regional anaesthesia to reduce drug use in anaesthesia and avoid aerosol generation. cochranelibrary.com/collections/doi/SC000041

\section{Acknowledgements}

We would like to acknowledge the outstanding commitment and invaluable role of our many volunteer authors, editors, and other contributors in supporting the efforts outlined in this report.

\section{Declarations of interest}

TQ is Managing Editor of Cochrane Emergency and Critical Care and Cochrane Anaesthesia. $\mathrm{HH}$ is Co-ordinating Editor of Cochrane Emergency and Critical Care. AS is Co-ordinating Editor of Cochrane Anaesthesia, and an editor for the journal Anaesthesia. AS has received research funding from the National Institute for Health Research and the European Society of Anaesthesiology. The authors declare no other competing interests.

\section{Funding}

Cochrane Emergency and Critical Care and Cochrane Anaesthesia receive core operational funding from the Danish Government and Capital Health Region.

\section{References}

1. Cochrane. News from the Cochrane Anaesthesia, Critical and Emergency Care Group. community.cochrane.org/news/newscochrane-anaesthesia-critical-and-emergency-care-group (accessed 9 October 2020).

2. Cochrane. Coronavirus (COVID-19): evidence relevant to critical care. cochranelibrary.com/collections/doi/SC000039 (accessed 9 October 2020).

3. Cochrane. Coronavirus (COVID-19): regional anaesthesia to reduce drug use in anaesthesia and avoid aerosol generation. cochranelibrary. com/collections/doi/SC000041 (accessed 9 October 2020)

4. Cumpstey AF, Oldman AH, Smith AF, Martin D, Grocott MP. Oxygen targets in the intensive care unit during mechanical ventilation for acute respiratory distress syndrome: a rapid review. Cochrane Database of Systematic Reviews 2020;(9):CD013708. https://doi. org/10.1002/14651858.CD013708

5. Cochrane Emergency and Critical Care. Summary of ongoing COVID-19 efforts. ec.cochrane.org/resources/summary-ongoing-covid19-efforts (accessed 9 October 2020).

6. Smith A. Promoting Cochrane evidence to the right audience Cochrane Dissemination Fellows. community.cochrane.org/news/ promoting-cochrane-evidence-right-audience-cochrane-disseminationfellows (accessed 9 October 2020).

7. Sng BL, Han NL, Leong WL, Sultana R, Siddiqui FJ, Assam PN, et al. Hyperbaric vs. isobaric bupivacaine for spinal anaesthesia for elective caesarean section: a Cochrane systematic review. Anaesthesia 2018;73(4):499-511. https://doi.org/10.1111/anae.14084 


\title{
REH-COVER (Rehabilitation-COVID-19 Evidence-based Response) action to recover functioning during/after COVID-19 and its treatments
}

\author{
Authors: Stefano Negrini ${ }^{1,2}$, Chiara Arienti ${ }^{3}$, Anne Cusick ${ }^{4}$, Carlotte Kiekens ${ }^{5}$, International Multiprofessional \\ Steering Committee of Cochrane Rehabilitation REH-COVER action \\ Participating groups: Cochrane Rehabilitation \\ Corresponding Author: Chiara Arienti: carienti@dongnocchi.it
}

\section{Introduction and background}

The headquarters of the Cochrane Rehabilitation (rehabilitation. cochrane.org) global network are in Italy, the first European country to enter lockdown, just two days after the World Health Organization (WHO) declared the COVID-19 pandemic. Suddenly, the whole world looked to Italy for the trajectory and impact of COVID-19.

Cochrane Rehabilitation responded with the REH-COVER (Rehabilitation - COVID-19 Evidence-based Response action. The aim was timely collection, review, and dissemination of synthesized evidence on rehabilitation for patients with COVID-19, their expected needs, and the impact of COVID-19 on people experiencing disabilities and on rehabilitation activities (Figure 1). The purpose of this article is to provide an account of the Cochrane Rehabilitation organizational response and the outcomes arising in publications, digital resources, and expertise networks.

\section{Key activities and strategies}

We augmented Cochrane Rehabilitation's usual arrangements with a dedicated group supporting the development and implementation of a response consistent with Cochrane Rehabilitation's purpose and scope. The name REH-COVER captured the rehabilitation, COVID-19, and evidence-based nature of the group's planned action. The REH-COVER international, multi-professional Steering Committee convened in March 2020 to provide advice, supervise all activities, and contribute to outcomes. Committee membership was drawn from four WHO regions and one low- to middle-income country, and included 13 experts (eight rehabilitation professions, epidemiologists, and one infectious disease specialist).

The REH-COVER programme of activities involved developing dedicated internet platforms and research publications for the dissemination of emerging and existing evidence about COVID-19 relevant to rehabilitation. Evidence was distinguished between low-level and gradually developing, but specific to COVID-19, and high-level but non-specific, coming from previous similar diseases.

Rehabilitation is a health strategy that aims to improve overall functioning of people experiencing disability with the potential of recovery or improvement.[1-3] 'Functioning' captures the impact of health conditions (including diseases and injuries) on body functions and structure, activities and participation and is proposed by WHO as a key health indicator alongside mortality and morbidity. $[4,5]$ In improving functioning, rehabilitation reduces disability, with broad health, social, and economic impacts.[6] Rehabilitation does not focus on one specific body function or structure but has a multi-professional, interdisciplinary approach to the whole person. $[7,8]$ Consequently, diverse health-research is needed to inform rehabilitation practice.

Cochrane Rehabilitation collaborates with the WHO rehabilitation programme (WHO-RP).[9] To structure the search and reporting of evidence, Cochrane Rehabilitation and WHO-RP together developed COVID-19-related research needs, including the following parameters: previous health conditions; COVID-19 phase (acute, post-acute, chronic, late-onset); functioning domains (impairments, activity limitations, participation restrictions, environmental factors, health services/policies, products/technology). Research

${ }^{1}$ University La Statale, Italy; ${ }^{2}$ sstituto Ortopedico Galeazzi, Milan, Italy; ${ }^{3}$ RCCS Fondazione Don Carlo Gnocchi, Milan, Italy; ${ }^{4}$ The University of Sydney, Australia; ${ }^{5}$ Spinal Unit, Montecatone Rehabilitation Institute, Imola, Italy 


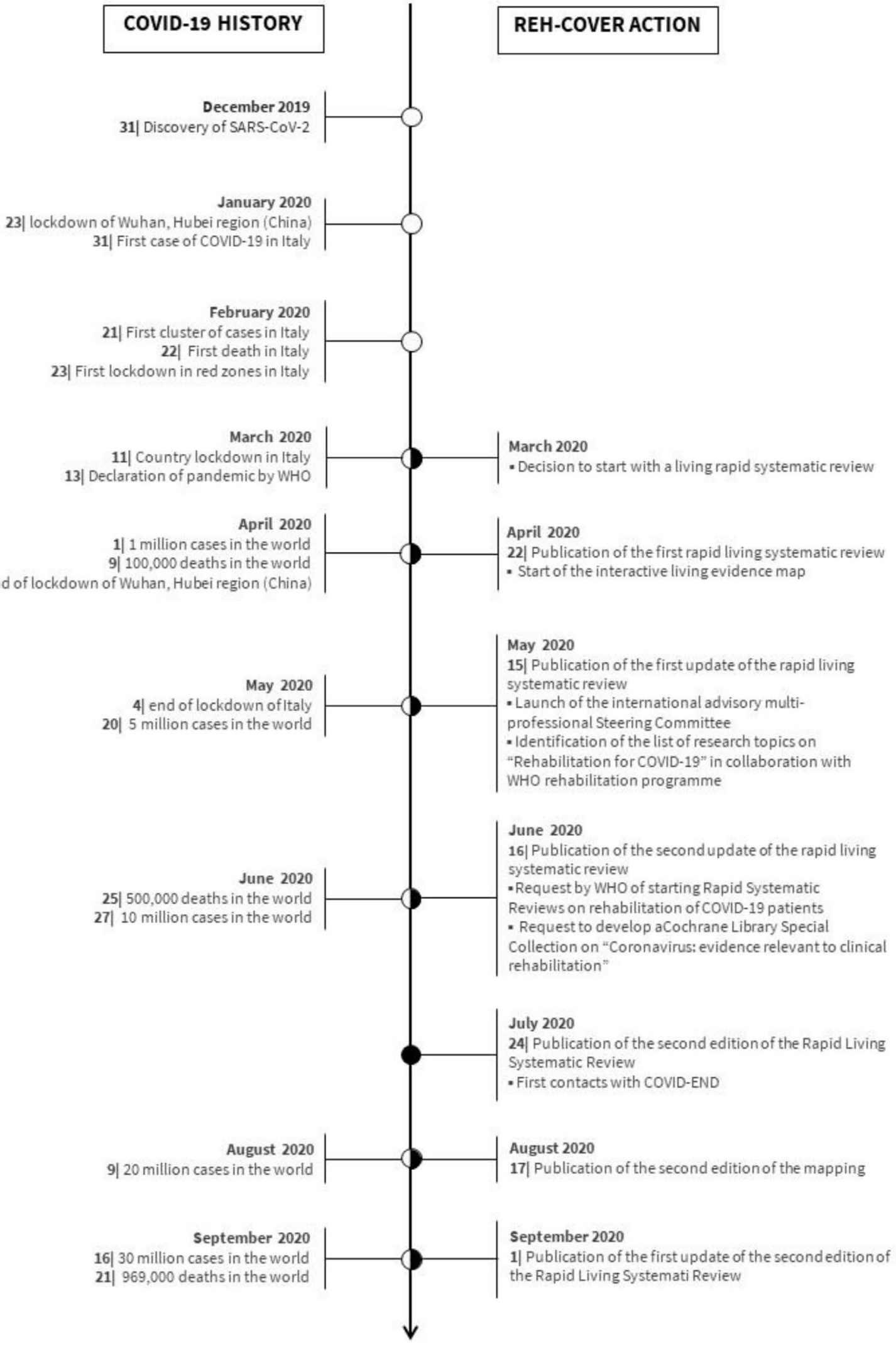

Figure 1. Timeline of development of Cochrane Rehabilitation REH-COVER (Rehabilitation - COVID-19 Evidencebased Response) action 
questions are informed by epidemiological, and micro- (individual), meso- (services) and macro- (health systems) level evidence.

\section{Outcomes and impact of activities}

We initiated a systematic review to address information needs. Since evidence was constantly growing, we used a rapid and living review methodology, so enabling constant update of results and methodology improvements. We published the first review in April 2020; two monthly updates followed, with a second, upgraded edition including Cochrane Rehabilitation/WHO-RP research questions.[10-14] We found low levels of evidence, and this will not change quickly because of the time-lag with rehabilitation following acute care,[1] and specific PICO problems, [3, 15-18] due to multiple comorbidities,[19] interventions,[20] 'usual' care, [21] and outcomes.[22.23] The first two published rapid living systematic reviews have already received nine Scopus citations in five months. $[10,11]$

The interactive living evidence map (currently in its second edition) has three formats: evidence map by research question and expected health impact; interactive table reporting results of the rapid living systematic reviews; and a geographic map showing where evidence was produced.

To collate and disseminate existing evidence relevant to rehabilitation needs of individuals who experienced COVID-19, we used epidemiological knowledge regarding disease sequelae,[13,14] and knowledge of adverse events of often invasive treatments, which can necessitate rehabilitation. Cochrane and most research indexing systems use disease categories. We thus identified relevant health conditions using an expert panel consensus process, in collaboration with WHO-RP and according to the current epidemiology.[13,14] We identified the following disorders: acute respiratory distress and pulmonary restrictive syndromes; postintensive care syndrome; swallowing disorders due to cranial nerve damage and post-extubation; multiple organ failure and shock; and post-traumatic stress disorder (PTSD). These topics served to start a series of rapid systematic reviews requested by WHO-RP and now under way.

We have developed a COVID-19 Cochrane Library Special Collection on the same topics. Cochrane Mental Health and Neurosciences Network identified PTSD reviews, which Cochrane Rehabilitation selected. Cochrane Rehabilitation developed an iterative survey process to prioritize the Cochrane reviews systematically searched on the other expected sequelae. Forty-eight Cochrane stakeholders' representatives were involved from the REH-COVER Steering Committee and Cochrane Rehabilitation Advisory Board, identifying seven reviews now included in the upcoming Special Collection, while five others were recommended for update.

\section{Lessons for the future: sustainability and transferability}

Rehabilitation and functioning can be secondary considerations in acute health settings where the primary concern is sustaining life and minimizing adverse biomedical events.[24] In a pandemic, the scope and scale of population impacts forces a public health approach that goes beyond acute care. Rehabilitation is an essential element of public health.[25] Long-COVID-19 discussion highlighted that concerns about mortality and morbidity were not enough - functioning, as a core aspect of health was also important.[26] The pandemic response reinforces the need to prioritize rehabilitation in health systems so all aspects of health are addressed.[4,9] COVID-19 provided the catalyst to reaffirm the central place of rehabilitation in population health responses to pandemic emergencies. COVID-19 also provided the platform to enhance Cochrane Rehabilitation efforts by: building on existing research knowledge (mapping, living and rapid reviews); leveraging the global Cochrane Rehabilitation expert network; responding to WHO calls for a collaborative evidence based response; delivering trustworthy appraised evidence; and maintaining and enhancing the Cochrane reputation in rehabilitation.

\section{Additional resources}

- REH-COVER (Rehabilitation - COVID-19 Evidence-based Response) action: rehabilitation.cochrane.org/resources/cochranerehabilitation-versus-covid-19

- International multiprofessional REH-COVER action Steering Committee: rehabilitation.cochrane.org/resources/reh-cover-action/ international-multiprofessional-steering-committee

- Rapid Living Systematic Reviews: rehabilitation.cochrane.org/ covid-19/reh-cover-rapid-living-systematic-reviews

- Living interactive evidence mapping: rehabilitation.cochrane.org/ covid-19/reh-cover-interactive-living-evidence

- Cochrane presentation of REH-COVER action: cochrane.org/news/ cochrane-rehabilitation-reh-cover-rehabilitation-covid-19-evidencebased-response-action

\section{Acknowledgements}

The International Multiprofessional Steering Committee of Cochrane Rehabilitation REH-COVER (REHabilitation for COVid-19: an Evidencebased Response) action includes the following contributors: Carlotte Kiekens (Montecatone Rehabilitation Institute, Italy); Chiara Arienti (IRCCS Fondazione Don Carlo Gnocchi, Italy); Maria Gabriella Ceravolo (Politecnica delle Marche University, Italy); Pierre Côté (Ontario Tech University, Canada); Anne Cusick (The University of Sydney, Australia); Francesca Gimigliano (University of Campania Luigi Vanvitelli, Italy); Allen Heinemann (Northwestern University Feinberg School of Medicine, and Centre for Rehabilitation Outcomes Research, Shirley Ryan AbilityLab, USA); Jody-Anne Mills (World Health Organization, Switzerland; University of Sydney, Australia); Farooq Rathore (PNS Shifa Hospital, Pakistan); Marco Rizzi (ASST Papa Giovanni XXIII Hospital, 
Italy); Geert Verheyden (University of Leuven, Belgium); Margaret Walshe (Trinity College Dublin, Ireland); Stefano Negrini (University La Statale, Italy; Istituto Ortopedico Galeazzi, Italy).

We wish to acknowledge the Cochrane Rehabilitation Headquarters (Roberta Bettinsoli, Stefano Giuseppe Lazzarini, Michele Patrini) and the Cochrane Rehabilitation Advisory Committee (Julia Patrick Engkasan, Francesca Gimigliano, Frane Grubisic, Elena Ilieva, William Levack, Thorsten Meyer, Aydan Oral, Farooq Rathore).

\section{Declarations of interest}

$\mathrm{SN}$ is Director of Cochrane Rehabilitation. He has a stock of the rehabilitation institute ISICO (Italian Scientific Spine Institute), Milan, Italy. CA is Co-ordinator of Cochrane Rehabilitation. CK is Co-Director of Cochrane Rehabilitation.

\section{Funding}

Cochrane Rehabilitation activities (including the REH-COVER action) are funded by: Fondazione Don Gnocchi, Milan, Italy; Istituto Ortopedico Galeazzi, Milan, Italy; Montecatone Rehabilitation Institute, Imola, Italy; International Society of Physical and Rehabilitation Medicine (ISPRM); European Society of Physical and Rehabilitation Medicine (ESPRM); European Union of Medical Specialists, Physical and Rehabilitation Medicine Section and Board.

\section{References}

1. European Physical and Rehabilitation Medicine Bodies Alliance. White Book on Physical and Rehabilitation Medicine in Europe: introductions, executive summary, and methodology. European Journal of Physical and Rehabilitation Medicine 2018;54(2):125-55. https://doi.org/10.23736/ S1973-9087.18.05143-2

2. Cieza A. Rehabilitation the health strategy of the 21st century, really? Archives of Physical Medicine and Rehabilitation 2019;100(11):2212-4. https://doi.org/10.1016/j.apmr.2019.05.019

3. Negrini S, Meyer T, Arienti C, Kiekens C, Pollock A, Selb M, et al. The 3rd Cochrane Rehabilitation Methodology Meeting: 'Rehabilitation definition for scientific research purposes'. European Journal of Physical and Rehabilitation Medicine 2020;Setember 16. https://doi. org/10.23736/S1973-9087.20.06574-0

4. Gimigliano F, Negrini S. The World Health Organization 'Rehabilitation 2030: a call for action'. European Journal of Physical and Rehabilitation Medicine 2017;53(2):155-68. https://doi.org/ 10.23736/s1973-

9087.17.04746-3

5. Heinemann AW, Feuerstein M, Frontera WR, Gard SA, Kaminsky LA, Negrini S, et al. Rehabilitation is a global health priority. European Journal of Physical and Rehabilitation Medicine 2020 56(2):129-30. https://doi.org/10.23736/S1973-9087.20.06284-X

6. European Physical and Rehabilitation Medicine Bodies Alliance. White Book on Physical and Rehabilitation Medicine in Europe. Chapter 2. Why rehabilitation is needed by individual and society. European Journal of Physical and Rehabilitation Medicine 2018;54(2):166-76. https://doi.org/ 10.23736/S1973-9087.18.05145-6
7. Zampolini M, Stucki G, Giustini A, Negrini S. The individual rehabilitation project: a model to strengthen clinical rehabilitation in health systems worldwide. European Journal of Physical and Rehabilitation Medicine 2020;56(1):1-4. https://doi.org/10.23736/S19739087.19.06110-0

8. European Physical and Rehabilitation Medicine Bodies Alliance. White Book on Physical and Rehabilitation Medicine (PRM) in Europe. Chapter 3. A primary medical specialty: the fundamentals of PRM. European Journal of Physical and Rehabilitation Medicine 2018;54(2):177-85. https://doi.org/10.23736/S1973-9087.18.05146-8

9. Rauch A, Negrini S, Cieza A. Toward strengthening rehabilitation in health systems: methods used to develop a WHO package of rehabilitation interventions. Archives of Physical Medicine and Rehabilitation 2019;100(11):2205-11. https://doi.org/10.1016/j. apmr.2019.06.002

10. Ceravolo MG, de Sire A, Andrenelli E, Negrini F, Negrini S. Systematic rapid 'living' review on rehabilitation needs due to COVID-19: update to March 31st, 2020. European Journal of Physical and Rehabilitation Medicine 2020;56(3):347-53. https://doi.org/10.23736/S19739087.20.06329-7

11. De Sire A, Andrenelli E, Negrini F, Negrini S, Ceravolo MG. Systematic rapid living review on rehabilitation needs due to COVID-19: update as of April 30th, 2020. European Journal of Physical and Rehabilitation Medicine 2020 56(3):354-60. https://doi.org/10.23736/S19739087.20.06378-9

12. Andrenelli E, Negrini F, de Sire A, Arienti C, Patrini M, Negrini S, et al. Systematic rapid living review on rehabilitation needs due to COVID-19: update to May 31st, 2020. European Journal of Physical and Rehabilitation Medicine 2020 56(4):508-14. https://doi.org/10.23736/ S1973-9087.20.06435-7

13. Ceravolo MG, Arienti C, De Sire A, Andrenelli E, Negrini F, Lazzarini S, et al. Rehabilitation and COVID-19: the Cochrane Rehabilitation 2020 rapid living systematic review. European Journal of Physical and Rehabilitation Medicine 2020; July 24 https://doi.org/10.23736/S19739087.20.06501-6

14. Negrini F, De Sire A, Andrenelli E, Lazzarini SG, Patrini M, Ceravolo MG, et al. Rehabilitation and COVID-19: the Cochrane Rehabilitation 2020 rapid living systematic review. Update as of July 31st, 2020. European Journal of Physical and Rehabilitation Medicine 2020. https:// doi.org/10.23736/S1973-9087.20.06539-9

15. Negrini S. Evidence in rehabilitation medicine: between facts and prejudices. American Journal of Physical Medicine \& Rehabilitation 2019;98(2):88-96.

16. Levack WM, Malmivaara A, Meyer T, Negrini S. Methodological problems in rehabilitation research. Report from a Cochrane Rehabilitation methodology meeting. European Journal of Physical and Rehabilitation Medicine 2019 55(3):319-21. https://doi.org/10.23736/ S1973-9087.19.05811-8

17. Negrini S, Meyer T, Arienti C, Malmivaara A, Frontera WR, Cochrane Rehabilitation Methodology Meeting participants. In search of solutions for evidence generation in rehabilitation: the second Cochrane 
Rehabilitation Methodology Meeting. American Journal of Physical Medicine \& Rehabilitation 2020;99(3):181-2. https://doi.org/10.1097/ PHM.0000000000001374

18. Negrini S, Levack W, Gimigliano F, Arienti C, Villafañe JH, Kiekens C. The struggle for evidence in physical and rehabilitation medicine: publication rate of randomized controlled trials and systematic reviews is growing more than in other therapeutic fields. American Journal of Physical Medicine \& Rehabilitation 2019;98(4):258-65. https://doi. org/10.1097/PHM.0000000000001058

19. Meyer T, Wulff K. Issues of comorbidity in clinical guidelines and systematic reviews from a rehabilitation perspective. European Journal of Physical and Rehabilitation Medicine 2019;55(3):364-71. https://doi. org/10.23736/S1973-9087.19.05786-1

20. Levack WM, Engkasan JP, Heinemann AW, Negrini S. A review of CONSORT guidelines about comparison groups with a focused discussion on implications for rehabilitation clinical trials. American Journal of Physical Medicine \& Rehabilitation 2020;99(3):191-7. https:// doi.org/10.1097/PHM.00000000000001368

21. Negrini S, Arienti C, Kiekens C. Usual care: the big but unmanaged problem of rehabilitation evidence. Lancet 2020;395(10221):337. https:// doi.org/10.1016/S0140-6736(19)32553-X

22. Stucki G, Pollock A, Engkasan JP, Selb M. How to use the International Classification of Functioning, Disability and Health as a reference system for comparative evaluation and standardized reporting of rehabilitation interventions. European Journal of Physical and Rehabilitation Medicine 2019 55(3):384-94. https://doi.org/10.23736/ S1973-9087.19.05808-8

23. Armijo-Olivo S, Dennett L, Arienti C, Dahchi M, Arokoski J, Heinemann AW, et al. Blinding in rehabilitation research: empirical evidence on the association between blinding and treatment effect estimates. American Journal of Physical Medicine \& Rehabilitation 2020;99(3):198-209. https://doi.org/10.1097/PHM.0000000000001377

24. Negrini S, Grabljevec K, Boldrini P, Kiekens C, Moslavac S, Zampolini $\mathrm{M}$, et al. Up to 2.2 million people experiencing disability suffer collateral damage each day of COVID-19 lockdown in Europe. European Journal of Physical and Rehabilitation Medicine 2020 56(3):361-5. https://doi. org/10.23736/S1973-9087.20.06361-3

25. World Health Organization. Report of the Global conference on primary health care: from Alma-Ata towards universal health coverage and the Sustainable Development Goals. January 2019. www.who. int/publications/i/item/report-of-the-global-conference-on-primaryhealth-care-from-alma-ata-towards-universal-health-coverage-and-thesustainable-development-goals

26. Wise J. Long COVID: doctors call for research and surveillance to capture disease. BMJ 2020;370:m3586. https://doi.org/10.1136/bmj. m3586 


\title{
COVID-19 SHORT REPORT
}

\section{COVID-19 Emergency Evidence Response Service: report from Ireland}

\author{
Authors: Nikita N Burke ${ }^{1,2,3}$, Sandra Galvin ${ }^{1,4}$, Declan Devane ${ }^{1,2,3,4}$, Ciara Keenan ${ }^{1,2,3}$ \\ Participating groups: Evidence Synthesis Ireland, Cochrane Ireland, Health Research Board Trials Methodology \\ Research Network, Cochrane COVID-19 Working Group, iHealthFacts.ie
}

Corresponding Author: Nikita Burke: nikita.burke@nuigalway.ie

\section{Introduction and background}

Evidence Synthesis Ireland (evidencesynthesisireland.ie), Cochrane Ireland (ireland.cochrane.org), and the Health Research Board Trials Methodology Research Network (hrb-tmrn.ie), were asked by the Irish Department of Health to focus our collective resources on prioritised evidence gaps for COVID-19 to support healthcare policy and practice decision-making. We harnessed existing connections and developed new collaborations with international colleagues and organisations to reduce duplication of effort, minimize research waste, share information, and ensure continued capacity building. The group, called the Emergency Evidence Response Service (EERS) worked quickly and flexibly on relevant questions that the World Health Organization (WHO), Cochrane, and ministries of health prioritized to be addressed using evidence synthesis skills.

The core EERS team, which was established at the National University of Ireland Galway (nuigalway.ie) on 15 March 2020, worked across more than 25 projects to address COVID-19 evidence gaps including infection prevention and control, impacts on healthcare and mental health, as well as supporting evidence dissemination, education, and advocacy.

\section{Key activities and strategies}

Working with networks including the Cochrane COVID-19 Working Group, led by Editor in Chief Karla Soares-Weiser, and the WHO Evidence Collaborative for COVID-19, we sought to support organizations that were answering critical questions and required evidence synthesis capacity. We provided experienced methodologists and systematic review authors, as well as information retrieval and peer-review expertise. We led on a number of reviews with support from the Cochrane Central Editorial Service (community.cochrane.org/review-production/ production-resources/cochranes-central-editorial-service).[1] We also developed iHealthFacts (ihealthfacts.ie) as a resource where the public can quickly and easily check the reliability of a health claim circulated by social media and thereby help tackle the 'infodemic' that continues to spread alongside COVID-19 in the wider community.

\section{Outcomes and impact of activities}

We were involved in four Cochrane COVID-19 reviews published to date, $[2,3,4]$ as well as a review of clinical practice guidelines, and curation of a special collection. We have published eight rapid reviews in total to date. The team participated in two Cochrane podcasts and two webinar conversations with the Editor in Chief. We collaborated with 11 centres (Figure 1 ) and had membership of three international COVID-19 networks. We featured in 21 media items, and placed eight volunteers with Evidence Aid (evidenceaid. org), who helped with screening, website support, and writing evidence summaries.

\section{Evidence Synthesis Ireland is leading on a priority-setting} partnership to establish the top 10 uncertainties in rapid review methodology, the Priority III project (evidencesynthesisireland.ie/ priority-iii). Though this project was planned and funded prior to the pandemic, its significance has been highlighted further with the explosion of rapid reviews during the COVID-19 crisis. Priority III aims to identify research priorities about how to improve how we plan, do and share the results of rapid reviews in the context of healthcare.

The study employs a priority-setting partnership based on the methods of the James Lind Alliance, which brings patients, carers and healthcare professionals together to identify and prioritise unanswered questions about healthcare that they jointly agree are the most important. We are partnering with COVID-END (covid-end.org),[13] and we are looking to recruit patient and public representatives, review authors, researchers, clinicians, or policymakers who are willing to share their views with us. There are

${ }^{1}$ National University of Ireland Galway, Ireland; ${ }^{2}$ Evidence Synthesis Ireland; ${ }^{3}$ Cochrane Ireland; ${ }^{4}$ Health Research Board Trials Methodology Research Network, Ireland 


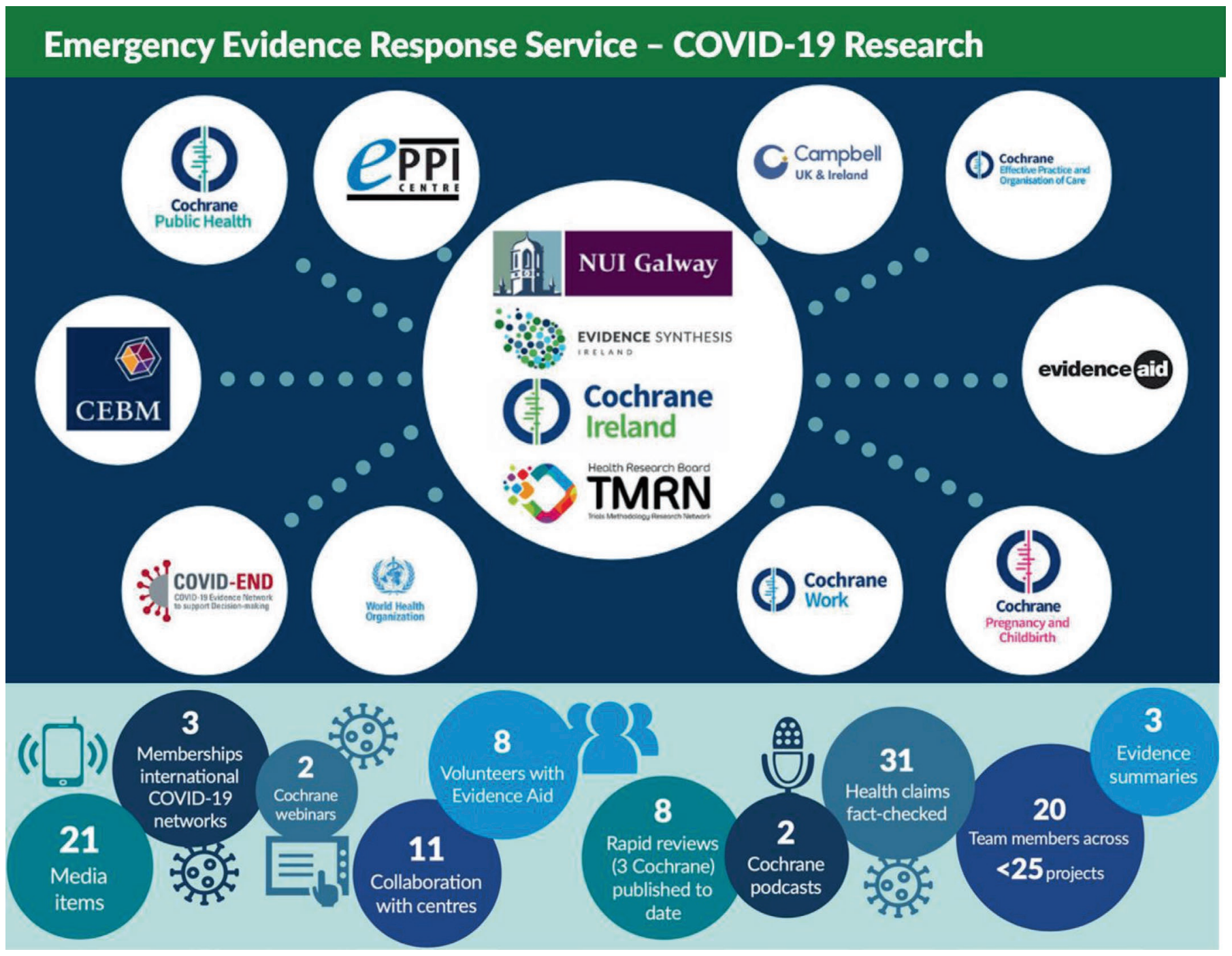

Figure 1. Emergency Evidence Response Service: COVID-19 research

three stages to the Priority III project, including two online surveys and a workshop.

Team members (Tom Conway, Ciara Keenan) are also working on a Cochrane Review (Cochrane Methodology) on interventions for retention in trials (led by Katie Gillies, Aberdeen, UK) and an EERS informational specialist (Mike Smalle) provided assistance with the search on the Cochrane Rapid Review (Cochrane Public Health) on digital solutions for contact tracing.[16]

We continued supporting dissemination and amplifying emerging evidence including sharing Cochrane Reviews and evidence in our newsletters, on social media, and producing three visual evidence summaries. Capacity building and education continues to be supported through Evidence Synthesis Ireland, Cochrane Ireland and the HRB Trials Methodology Research Network through a Special Symposium, (www.hrb-tmrn.ie/trainingeducation/2020-special-symposium-trials-in-a-pandemic) ESI webinars (evidencesynthesisireland.ie/resources), and funding COVID summer studentships and collating resources (evidencesynthesisireland.ie/covid-19).

iHealthFacts.ie (ihealthfacts.ie), a new health-claim fact-checking service for the public was developed within four weeks and has robust processes underpinning how we determine the reliability of health claims circulating on social media. This includes searching for systematic reviews of studies to help us inform decisions, independent review by a second researcher, a healthcare specialist and public representative, and a health journalist. We review claims continuously and a number have been updated and some, such as masks and ibuprofen, more than once. 
Table 1. Key reviews of the Emergency Evidence Response Service

\begin{tabular}{|c|c|c|c|c|}
\hline Type & Title & Group & Role & Reference \\
\hline $\begin{array}{l}\text { Cochrane Rapid } \\
\text { qualitative } \\
\text { evidence synthesis }\end{array}$ & $\begin{array}{l}\text { Barriers and facilitators to healthcare } \\
\text { workers' adherence with infection } \\
\text { prevention and control (IPC) guidelines } \\
\text { for respiratory infectious diseases: a rapid } \\
\text { qualitative evidence synthesis }\end{array}$ & Cochrane EPOC & Lead & Houghton 2020[2] \\
\hline $\begin{array}{l}\text { Cochrane Rapid } \\
\text { Review }\end{array}$ & $\begin{array}{l}\text { Video calls for reducing social isolation and } \\
\text { loneliness in older people }\end{array}$ & Cochrane Public Health & Lead & Noone 2020[3] \\
\hline $\begin{array}{l}\text { Cochrane Review } \\
\text { update }\end{array}$ & $\begin{array}{l}\text { Personal protective equipment for } \\
\text { preventing highly infectious diseases due } \\
\text { to contact with contaminated body fluids in } \\
\text { health care staff }\end{array}$ & Cochrane Work & $\begin{array}{l}\text { Co-authors } \\
\text { (Toomey, } \\
\text { Blackwood) }\end{array}$ & Verbeek 2020[4] \\
\hline $\begin{array}{l}\text { Review of clinical } \\
\text { practice guidelines }\end{array}$ & $\begin{array}{l}\text { COVID-19 review of clinical practice } \\
\text { guidelines for key questions relating to the } \\
\text { care of pregnant women and their babies }\end{array}$ & $\begin{array}{l}\text { Cochrane Pregnancy and } \\
\text { Childbirth }\end{array}$ & Lead & Devane 2020[5] \\
\hline $\begin{array}{l}\text { Cochrane Library } \\
\text { Special Collection }\end{array}$ & $\begin{array}{l}\text { Coronavirus (COVID-19): remote care } \\
\text { through telehealth }\end{array}$ & $\begin{array}{l}\text { Cochrane Editorial \& Methods } \\
\text { Department and others }\end{array}$ & Lead & Cochrane 2020[6] \\
\hline $\begin{array}{l}\text { Living mapping } \\
\text { and living } \\
\text { systematic review }\end{array}$ & $\begin{array}{l}\text { The COVID-NMA initiative: a living mapping } \\
\text { and living systematic review of Covid-19 } \\
\text { trials }\end{array}$ & $\begin{array}{l}\text { Cochrane France, } \\
\text { Cochrane Germany, } \\
\text { Cochrane Chile, and others }\end{array}$ & $\begin{array}{l}\text { Steering } \\
\text { Group, } \\
\text { mapping } \\
\text { (Devane, } \\
\text { Quirke) }\end{array}$ & Boutron 2020[7,8] \\
\hline $\begin{array}{l}\text { Rapid review of } \\
\text { reviews }\end{array}$ & $\begin{array}{l}\text { What remotely delivered interventions } \\
\text { can reduce social isolation and loneliness } \\
\text { among older adults? }\end{array}$ & EPPI-Centre & $\begin{array}{l}\text { Co-author } \\
\text { (Casey) }\end{array}$ & Boulton 2020[9] \\
\hline $\begin{array}{l}\text { Rapid evidence } \\
\text { review }\end{array}$ & $\begin{array}{l}\text { What is the efficacy of standard face masks } \\
\text { compared to respirator masks in preventing } \\
\text { cOVID-type respiratory illnesses in primary } \\
\text { care staff? }\end{array}$ & $\begin{array}{l}\text { Centre for Evidence-Based } \\
\text { Medicine }\end{array}$ & $\begin{array}{l}\text { Co-authors } \\
\text { (Devane, } \\
\text { Toomey) }\end{array}$ & $\begin{array}{l}\text { Greenhalgh } \\
2020[10]\end{array}$ \\
\hline $\begin{array}{l}\text { Rapid evidence } \\
\text { review }\end{array}$ & $\begin{array}{l}\text { What is the evidence that COVID- } 19 \text { personal } \\
\text { protective equipment should include shoe } \\
\text { covers? }\end{array}$ & $\begin{array}{l}\text { Centre for Evidence-Based } \\
\text { Medicine }\end{array}$ & $\begin{array}{l}\text { Co-authors } \\
\text { (Devane, } \\
\text { Toomey) }\end{array}$ & Khunti 2020[11] \\
\hline $\begin{array}{l}\text { Rapid evidence } \\
\text { review }\end{array}$ & $\begin{array}{l}\text { What is the efficacy of eye protection } \\
\text { equipment in primary care settings? }\end{array}$ & $\begin{array}{l}\text { Centre for Evidence-Based } \\
\text { Medicine }\end{array}$ & $\begin{array}{l}\text { Co-authors } \\
\text { (Devane, } \\
\text { Toomey) }\end{array}$ & Khunti 2020[12] \\
\hline $\begin{array}{l}\text { Rapid evidence } \\
\text { review }\end{array}$ & $\begin{array}{l}\text { What is the performance and impact } \\
\text { of disposable and reusable respirators } \\
\text { for healthcare workers in the context of } \\
\text { COVID-19? }\end{array}$ & $\begin{array}{l}\text { Centre for Evidence-Based } \\
\text { Medicine }\end{array}$ & $\begin{array}{l}\text { Co-author } \\
\text { (Toomey) }\end{array}$ & Burton 2020[13] \\
\hline $\begin{array}{l}\text { Rapid evidence } \\
\text { review }\end{array}$ & $\begin{array}{l}\text { Extended use or re-use of single-use surgical } \\
\text { masks and filtering facepiece respirators: a } \\
\text { rapid evidence review }\end{array}$ & $\begin{array}{l}\text { Centre for Evidence-Based } \\
\text { Medicine }\end{array}$ & $\begin{array}{l}\text { Lead (Toomey, } \\
\text { Conway Y) }\end{array}$ & Toomey 2020[14] \\
\hline
\end{tabular}

Since its launch on 7 April 2020, the iHealthFacts website has had:

- 35,000 unique page views from over 90 countries to date (September 2020);
- 500 claims for iHealthFacts to research submitted by the public with over 1000 searches on the website;

- 31 claims answered on the website with five pending publication; claims answered include evidence for the treatment 
or prevention of COVID-19 with Vitamin C and D, chloroquine; or preventing the spread of COVID-19 through disinfecting food packaging, wearing masks or gloves, and more;

- 351,000 Twitter impressions, 3500 impressions on Instagram and Facebook posts reaching over 10,000 people.

iHealthFacts featured in seven newspapers including interviews in The Irish Times and The Sunday Times and recorded six interviews, including on primetime national radio. We believe it has become a valuable part of the Irish response to COVID-19 as well as addressing a need to counter harmful health misinformation and teach critical thinking skills.

\section{Lessons for the future: sustainability and transferability}

The overall ability for the EERS to be established at such a rapid pace and deliver numerous outputs to such high standards lay in the underlying national support infrastructures, funded by the Health Research Board (HRB-TMRN EUR 2.6 million since 2015) and funding from both the Health Research Board and the Health and Social Care, Research and Development Division of the Public Health Agency in Northern Ireland to ESI and Cochrane Ireland (almost EUR 2.0 million in December 2018).

What this support demonstrated during COVID-19 was a firm foundation of expertise, experience and dedicated personnel, with an excellent knowledge of the research landscape in Ireland, to be able to mount an agile and innovative response to achieve the work required. Not only did this ensure impact in Ireland, it put Ireland on the global COVID-19 map, contributing to an international response.

These infrastructures have worked to ensure their organizational brands are a trusted and reliable source for research activities, ensuring the highest operational standards when conducting and publishing research. This combination of established organizations meant that the EERS was planned, launched and operational in days, with outputs visible in weeks. Key assistance came from NUI Galway core supports and our funders to ensure the rapidity of response that the health emergency required. None of this would have been possible without outstanding team work, cohesiveness, goodwill, talent, capacity and leadership from the researchers, collaborators and volunteers who produced the work with enormous effort, time and commitment. The establishment of the EERS during this pandemic has now built strong networks and a reputation as being a source of robust and reliable evidence and methodologists available for future pandemics.

\section{Additional resources}

- Evidence Synthesis Ireland: evidencesynthesisireland.ie

- Priority III - rapid reviews project: evidencesynthesisireland.ie/ priority-iii

- Cochrane Ireland: ireland.cochrane.org
- HRB Trials Methodology Research Network: www.hrb-tmrn.ie

- iHealthFacts: ihealthfacts.ie

- Webinars: evidencesynthesisireland.ie/resources

- More information: evidencesynthesisireland.ie/covid-19

\section{Acknowledgements}

- EERS members (NUI Galway): Claire Beecher, Linda Biesty, Nikita Burke, Dympna Casey, Tom Conway, Yvonne Conway, Hannah Delaney, Declan Devane, Elaine Finucane, Sandra Galvin, Vivienne Hanrahan, Catherine Houghton, Fionnuala Jordan, Ciara Keenan, Chris Noone, Jenny McSharry, Eimear Morrissey, Mike Smalle, Elaine Toomey, Fiona Quirke.

- The iHealthFacts team

- NUI Galway contributors: Maureen Kelly, Ciara Gleeson, Simone LePage, Sirisha Bellamkonda, Hema Sivasubramanian, Sravya Pudota, Nikolette Warner

- NUI Galway supporters; Carol Burke, Sheena Connolly, Ann Melia, Ciarán Ó hÓgartaigh, Tim O'Brien, Sharon Bailey, Josephine Hynes, Niamh Connolly, Caroline Loughnane, Martina Ní Chúlain

- With thanks to Teresa Maguire, the Health Research Board and the HSC R\&D Public Health Agency

- Organizations: Cochrane (Cochrane Central Executive Team, Cochrane Public Health, Cochrane Effective Practice and Organisation of Care, Cochrane Work, Cochrane Pregnancy and Childbirth), World Health Organization (WHO), Campbell UK \& Ireland, EPPI-Centre (London), the Centre for Evidence Based Medicine (Oxford), and COVID-19 Evidence Network to support Decision-making (COVID-END)

- Specific acknowledgements are described directly in the individual publications.

\section{Declarations of interest}

The authors are paid in full, or part, from Evidence Synthesis Ireland, which is a capacity-building initiative funded by the Health Research Board and HSC R\&D Public Health Agency, Northern Ireland funding to Evidence Synthesis Ireland and Cochrane Ireland (CBES-2018-001) (to NB, DD, CK) or from the HRB Trials Methodology Research Network (SG).

\section{Funding}

Health Research Board (HRB) funding to the HRB-TMRN (HRBTMRN-2017-1), Health Research Board and HSC R\&D Public Health Agency, Northern Ireland funding to Evidence Synthesis Ireland and Cochrane Ireland (CBES-2018-001); and HRB funding for the Membrane sweeping for induction of labour: The MILO Study (DIFA-2018-005).

\section{References}

1. Wakeford H, Dooley C, Stephani A-M, Marshall R, Featherstone R, Walshe D, et al. The Central Editorial Service: a collaborative editorial process for publishing high-priority Cochrane reviews. In: Collaborating in response to COVID-19: editorial and methods initiatives across Cochrane. Cochrane Database of Systematic Reviews 2020;(12 Suppl 1). https://doi.org/10.1002/14651858.CD202002 
2. Houghton C, Meskell P, Delaney H, Smalle M, Glenton C, Booth $A$, et al. Barriers and facilitators to healthcare workers' adherence with infection prevention and control (IPC) guidelines for respiratory infectious diseases: a rapid qualitative evidence synthesis. Cochrane Database of Systematic Reviews 2020;(4):CD013582. https://doi. org/10.1002/14651858.CD013582

3. Noone C, McSharry J, Smalle M, Burns A, Dwan K, Devane D, et al. Video calls for reducing social isolation and loneliness in older people: a rapid review. Cochrane Database of Systematic Reviews 2020;(5):CD013632. https://doi.org/10.1002/14651858.CD013632

4. Verbeek JH, Rajamaki B, ljaz S, Sauni R, Toomey E, Blackwood B, et al. Personal protective equipment for preventing highly infectious diseases due to exposure to contaminated body fluids in healthcare staff. Cochrane Database of Systematic Reviews 2020;(4):CD011621. https:// doi.org/10.1002/14651858.CD011621.pub4

5. Devane D, Alfirevic Z, Kellie F, Finucane E, Beecher C, Hanrahan V, et al. Review of clinical practice guidelines for the care of pregnant women and their babies during COVID-19. 2020. pregnancy.cochrane.org/news/ covid-19-review-national-clinical-practice-guidelines-key-questionsrelating-care-pregnant

6. Cochrane. Coronavirus (COVID-19): remote care through telehealth. 2020. www.cochranelibrary.com/collections/doi/SC000043

7. Boutron I, Chaimani A, Meerpohl JJ, Hróbjartsson A, Devane D, Rada G. et al. The COVID-NMA Project: building an evidence ecosystem for the COVID-19 pandemic. Annals of Internal Medicine 2020;September 15. https://doi.org/10.7326/M20-5261

8. Boutron I, Tovey DI, De Nale L, Chaimani A, Devane D, Meerpohl JJ, et al. COVID-NMA: a collaborative COVID-19 living evidence project. In: Collaborating in response to COVID-19: editorial and methods initiatives across Cochrane. Cochrane Database of Systematic Reviews 2020;(12 Suppl 1). https://doi.org/10.1002/14651858.CD202002

9. Boulton E, Kneale D, Stansfield C, Heron P, Sutcliffe K, Hayanga B, et al. Rapid review of reviews: what remotely delivered interventions can reduce social isolation and loneliness among older adults? 2020. EPPICentre. Available at eppi.ioe.ac.uk/cms/Default.aspx?tabid=3770

10. Greenhalgh T, Chan X-H, Khunti K, Durand-Moreau Q, Straube S, Devane $\mathrm{D}$, et al. What is the efficacy of standard face masks compared to respirator masks in preventing COVID-type respiratory illnesses in primary care staff? 24 March 2020. cebm.net/covid-19/what-is-theefficacy-of-standard-face-masks-compared-to-respirator-masks-inpreventing-covid-type-respiratory-illnesses-in-primary-care-staff

11. Khunti K, Greenhalgh T, Chan X-H, Durand-Moreau Q, Straube S, Devane $\mathrm{D}$, et al. What is the evidence that COVID-19 personal protective equipment should include shoe covers? 7 April 2020. cebm.net/ covid-19/what-is-the-evidence-that-covid-19-personal-protectiveequipment-should-include-shoe-covers

12. Khunti K, Greenhalgh T, Chan X-H, Durand-Moreau Q, Straube $S$, Devane $D$, et al. What is the efficacy of eye protection equipment compared to no eye protection equipment in preventing transmission of COVID-19-type respiratory illnesses in primary and community care? 3 April 2020. cebm.net/covid-19/what-is-the-efficacy-of-eye-protectionequipment-compared-to-no-eye-protection-equipment-in-preventingtransmission-of-covid-19-type-respiratory-illnesses-in-primary-andcommunity-care

13. Burton C, Coles B, Adisesh A, Smith S, Toomey E, Chan X-H, et al. What is the performance and impact of disposable and reusable respirators for healthcare workers in the context of COVID-19? 22 May 2020. cebm.net/covid-19/what-is-the-performance-and-impact-ofdisposable-and-reusable-respirators-for-healthcare-workers-in-thecontext-of-covid-19

14. Toomey E, Conway Y, Burton C, Smith S, Smalle M, Chan X-H, et al. Extended use or re-use of single-use surgical masks and filtering facepiece respirators: a rapid evidence review. 3 June 2020. cebm.net/ covid-19/extended-use-or-re-use-of-single-use-surgical-masks-andfiltering-facepiece-respirators-a-rapid-evidence-review

15. Grimshaw JM, Tovey DI, Lavis JN, on behalf of COVID-END. COVIDEND: an international network to better co-ordinate and maximize the impact of the global evidence synthesis and guidance response to COVID-19. In: Collaborating in response to COVID-19: editorial and methods initiatives across Cochrane. Cochrane Database of Systematic Reviews 2020;(12 Suppl 1). https://doi.org/10.1002/14651858.CD202002

16. Anglemyer A, Moore TH, Parker L, Chambers T, Grady A, Chiu K, et al. Digital contact tracing technologies in epidemics: a rapid review. Cochrane Database of Systematic Reviews 2020;(8):CD013699. https:// doi.org/10.1002/14651858.CD013699 


\title{
Collaboration in times of Coronavirus: reflections on a living systematic review of COVID-19 in pregnancy
}

\author{
Authors: Elena Stallings ${ }^{1,2,3}$, John Allotey ${ }^{4,5}$, Madelon van Wely ${ }^{6}$, Shakila Thangaratinam ${ }^{5,7}$, Javier Zamora ${ }^{1,2,3,5}$ for \\ the PregCOV collaboration \\ Participating groups: Cochrane Gynaecology and Fertility: Netherlands Satellite, WHO Collaborating Centre \\ for Global Women's Health, University of Birmingham, Cochrane Madrid, CIBER Epidemiology and Public Health \\ (CIBERESP), Guangzhou Women and Children's Medical Centre, WHO Human Reproduction Programme, Elizabeth \\ Glaser Paediatric AIDS Foundation, Queen Mary University of London, St Georges University, UK
}

Corresponding Author: Javier Zamora: javierza@gmail.com

\section{Introduction and background}

Coronavirus disease 2019 (COVID-19) was officially declared a pandemic by the World Health Organization (WHO) in March 2020. COVID-19 is especially dangerous for at-risk populations, such as pregnant women,[1] thus making it critical to determine how COVID-19 affects them and their babies. A regular systematic review methodology would not be sufficient to synthesize the overwhelming amount of evidence produced daily worldwide. We needed to carry out a living systematic review (LSR), meaning that the review would be continually updated, incorporating new studies as they become available.[2]

The PregCOV-19 living systematic review working group was quickly established through an international collaboration, which included researchers and medical students at the University of Birmingham, UK; the World Health Organization; the Cochrane Gynaecology and Fertility Netherlands Satellite; and researchers in other parts of the UK, Spain (Cochrane Madrid), China, and the USA. This project commenced at the beginning of April 2020, just when the pandemic was gathering full force around Europe. At that point we were in a full lockdown, working from home, and online meetings had quickly become the new normal.

\section{Key activities and strategies}

We developed a protocol for the project that encompassed numerous clinical questions. For the LSR that we published in September 2020, we took the usual systematic review steps. We carried out rigorous searches on a weekly basis in the major medical databases for studies relating to COVID-19 in pregnant women. We screened thousands of studies $(49,684)$ for inclusion in our review. [3] We also extracted data and carried out quality assessment on a weekly basis. At first we carried out statistical analysis every two weeks, however once we saw the results were not varying greatly, the analysis moved to monthly, and then bi-monthly.

In order to co-ordinate this process, we had weekly team meetings, which have now moved to biweekly. Throughout the review process we were also constantly adding new members to the team. The new team members go through a training process, in which they shadow other team members, for one to two weeks before carrying out the work independently.

We created a website (birmingham.ac.uk/research/whocollaborating-centre/pregcov), linked to the University of Birmingham, to highlight the project and make it easily accessible to pregnant women, researchers, and clinicians worldwide. We are currently updating the results on the website every two months.

\section{Outcomes and impact of activities}

We published the PregCOV19 project protocol on PROSPERO in April 2020.[4] The aim of this project is to assess the impact of COVID-19 in pregnancy. From this protocol we have several different studies planned. The first study was a LSR, fast-tracked by the $B M J$, entitled 'Clinical manifestations, risk factors, and maternal and perinatal outcomes of coronavirus disease 2019 in pregnancy: living systematic review and meta-analysis'. The review took only

${ }^{1}$ Hospital Universitario Ramón y Cajal (IRYCIS), Spain; ${ }^{2}$ CIBER Epidemiology and Public Health (CIBERESP), Spain; ${ }^{3}$ Cochrane Associate Centre of Madrid, Spain; ${ }^{4}$ University of Birmingham, UK; ${ }^{5} \mathrm{WHO}$ Collaborating Centre for Global Women's Health, University of Birmingham, UK; ${ }^{6} \mathrm{Netherlands} \mathrm{Satellite} \mathrm{of}$ Cochrane Gynaecology and Fertility, Amsterdam University Medical Center, The Netherlands; 'Birmingham Women's and Children's NHS Foundation Trust, UK 
five months from initiation to publication in the $B M J$, peer-review process included, while most systematic reviews take years to complete and then be published. During public health crises, the best evidence needs to be peer-reviewed and made available to medical professionals and the public immediately. That is why a living systematic review format is the best option during times of crisis such as a pandemic.[5] Future updates with additional studies will allow us to confirm our results with a higher level of certainty.

One of our significant findings so far is that pregnant and recently pregnant women may be at increased risk of admission to an intensive care unit (ICU).[3] This finding led to a lot of media interest, with the published LSR being reported by various news outlets worldwide, such as CNN, The Guardian, and Bloomberg. We hope that it will inform clinical guidelines and practice on the management of pregnant women with COVID-19.

\section{Lessons for the future: sustainability and transferability}

This project proved challenging on a few fronts. The first challenge was the pace we were working at. There were many late nights, which turned into early mornings endured by all the team. Just when we thought we were finished, the next influx of data would come rolling in and we would start the process again. It is difficult to maintain the pace that we have been working at for the past five months. During the lockdown we pushed aside other commitments and projects to dedicate nearly all our time to this project. This is not feasible moving forward. We must start dividing our time among our other various tasks again. We are also losing a lot of valuable team members as the medical students transition back to classes. So, going forward into the second wave of the virus, we must be conscious of these new limitations we face with the team.

The second challenge, albeit an opportunity, is that we were such a large group, collaborating from many different countries around all points of the globe. Frequent zoom meetings were a must, although sometimes a struggle co-ordinating different time zones. However, this also allowed us to have clinicians, statisticians, epidemiologists and students working in our team, which helped keep it diverse and see all angles of the problem.

We have created a basic framework for a large-scale LSR that can answer multiple research questions. This research framework can be deployed in the future for other public health crises or future pandemics. The way we have organized the team, each member carrying out their specific duties every week, makes all the systematic review stages tick on without fault. The data extraction sheets are organized in an Excel workbook for all the various review questions, varying from prevalence to risk factors. This format makes it easily transferable to other projects as an LSR framework in the future.

\section{Links to additional resources}

- The LSR published in the BMJ: doi.org/10.1136/bmj.m3320

- The PregCOV19 website: birmingham.ac.uk/research/whocollaborating-centre/pregcov/index.aspx

- LSR protocol: crd.york.ac.uk/prospero/display_record. php?ID=CRD42020178076

\section{Acknowledgements}

We would like to thank all of the members of the PregCOV team for all of their hard work on this project, especially the medical students from the University of Birmingham.

\section{Declarations of interest}

Madelon Van Wely leads the Netherlands Satellite of Cochrane Gynaecology and Fertility. Elena Stallings and Javier Zamora are members of the Cochrane Associate Centre Madrid.

\section{Funding}

The project was partially funded by the World Health Organization.

\section{References}

1. Centers for Disease Control and Prevention. People at increased risk and other people who need extra precautions [updated 21 July 2020]. Available at cdc.gov/coronavirus/2019-ncov/need-extra-precautions/ index.html

2. Elliott JH, Synnot A, Turner T, Simmonds M, Akl EA, McDonald S, et al. Living systematic review: 1 . Introduction-the why, what, when, and how. Journal of Clinical Epidemiology 2017;91:23-30. https://doi.org/ 10.1016/j.jclinepi.2017.08.010

3. Allotey J, Stallings E, Bonet M, Yap M, Chatterjee S, Kew T, et al. Clinical manifestations, risk factors, and maternal and perinatal outcomes of coronavirus disease 2019 in pregnancy: living systematic review and meta-analysis. BMJ 2020;370:m3320. https://doi. org/10.1136/bmj.m3320

4. Allotey J, Bonet M, Stallings E, Yap M, Chatterjee S, Kew T, el al. COVID-19 in pregnant women: a living systematic review on prevalence, presentation, prognosis and treatment. PROSPERO 2020 CRD42020178076 Available from: https://www.crd.york.ac.uk/prospero/ display_record.php?ID=CRD42020178076

5. Turner T, McDonald S. Cochrane COVID-19 living systematic reviews: why, how, and what has been done? In: Collaborating in response to COVID-19: Editorial and methods initiatives across Cochrane. Cochrane Database of Systematic Reviews 2020;(11 Suppl 1). https://doi. org/10.1002/14651858.CD202002 


\title{
Lessons in providing rapid evidence to inform national treatment guidelines for COVID-19 in South Africa
}

\author{
Authors: Amanda Brand ${ }^{1}$, Ameer Hohlfeld ${ }^{2}$, Michael McCaul ${ }^{1}$, Solange Durão², Taryn Young ${ }^{1}$, Tamara Kredo ${ }^{2}$ \\ Participating groups: Cochrane South Africa, South African GRADE Network, Centre for Evidence Based Health \\ Care, Stellenbosch University
}

Corresponding Author: Amanda Brand: asbrand@sun.ac.za

\section{Introduction and background}

When the severe acute respiratory syndrome coronavirus 2 (SARS CoV-2) hit, there were no established prevention or treatment protocols.[1] High-quality evidence was urgently needed.[2] An explosion of studies and reviews globally - often with considerable duplication, variable quality and sometimes contradictory findings - added to an unprecedented 'infodemic'.[3] As a result, the overall picture of the evidence base was, and remains, subject to change as data emerge.

Given this context, the South African National Department of Health's (NDoH) National Essential Medicine List Committee (NEMLC) COVID-19 sub-committee was established to review evidence and inform national guidance. However, the critical nature of the health emergency, and the speed of evidence production, required a turn-about in the usual, relatively slow processes for guideline decision making. Thus, the health crisis enabled the development of a rapid review process to balance urgency and rigorous methods, based on Cochrane's evolving rapid review methodology, while generating the highest possible quality of evidence.[4] The NEMLC sub-committee, with the support of the South African (SA) GRADE Network, piloted this rapid review approach through iterative real-time collaboration; to minimize time lost in planning and ensure a timely response.

Establishment of a COVID-19 responsive evidence service is not unique to South Africa. Many lead evidence synthesis, guideline and health technology assessment organizations sprang into action to establish similar activities. Some examples include the UK Oxford Evidence Service (cebm.net/oxford-covid-19-evidenceservice) and the Australian National Covid-19 Clinical Evidence Taskforce (covid19evidence.net.au/\#living-guidelines), to name just two. Aspects of our work reflect those of global organizations, such as the use of systematic methods and including GRADE and transparency in decision-making. However, there are three important differences in our approach worth mentioning.

1. There are insufficient numbers of technically skilled researchers and methodologists in South Africa to rapidly engage to conduct reviews.

2. In South Africa, like everywhere, evidence regarding the best available prevention and treatment for COVID-19 was urgently required, however, we faced other grave public health issues that required an evidence-informed response, such as the safety of public transport, COVID-19 lockdowns and violence, alcohol bans, and economic sustainability and food security.

3. Finally, given the limited technical resources, our approach heeded the call to minimize duplication of efforts by finding and using available, current and high-quality systematic reviews to inform national decisions. This process of finding and using what is available first is reflected in the algorithm developed for COVID-END evidence synthesis group with involvement from Taryn Young (mcmasterforum.org/networks/covid-end).[5]

\section{Key activities and strategies}

Members of the NEMLC COVID-19 sub-committee were paired with systematic review authors from the SA GRADE Network. These teams aimed to complete a review within 7 to 10 days from receipt of the guideline question from the NEMLC sub-committee. The teams developed a search strategy, drawing on search terms from existing systematic reviews where available, and searched three electronic databases: Epistemonikos (epistemonikos.org); Cochrane COVID-19 Study Register (covid-19.cochrane.org); and the COVIDNMA initiative (www.covid-nma.com). We carried out screening in duplicate, using Covidence,[6] and resolved discrepancies through consensus. We assessed quality of included systematic reviews in 
duplicate using AMSTAR 2. A single review author extracted data, which were independently checked by a second review author.

To ensure efficiency, we used results from existing high-quality systematic reviews to inform the pre-defined outcomes. Where these were not available or did not report on an outcome of interest, we used evidence preferentially according to the evidence hierarchy, that is, starting with RCTs.[7] If the quality of the evidence had already been appraised and 'GRADE-ed', for example, for the living mapping and living systematic review of COVID-19 studies (www.covid-nma.com), we used these resources with necessary acknowledgement to minimize delays.[8] In cases where quality appraisals for primary studies were not available, the we assessed the evidence using Cochrane's Risk of Bias 2 (RoB 2) tool,[9] and GRADEpro GDT.[10]

These activities informed the draft of the methods and results sections of the rapid reviews. Committee members from the NEMLC COVID-19 sub-committee then structured their conclusions and recommendations, using the GRADE Evidence to Decision (EtD) Framework. The report was circulated to the full sub-committee for input before final decisions were ratified.

\section{Outcomes and impact of activities}

Developing and conducting these rapid reviews resulted in:

1. recommendations for 11 potential treatments and three potential prophylactics;

2. development of methods guides and reporting templates, matching the needs of this $\mathrm{NDoH}$ group, for use for future rapid reviews;

3. the 'proof of concept' for an efficient workflow to conduct reviews with support from SA GRADE review authors;

4. learning and skills-building among members of the SA GRADE Network to conduct rapid reviews, which are timely and relevant for use by South African policymakers;

5. learning, collaboration and skills-building among members of the NEMLC to understand and contribute to GRADE and guideline development processes;

6. greater linkages and strengthened relationships between the NDoH, SA GRADE Network and other stakeholders; and

7. evidence-informed national guidelines.

\section{Lessons for the future: sustainability and transferability}

The crucial lesson learnt is not to reinvent the wheel and to reduce duplication. Our teams tapped into available resources, particularly Cochrane-linked resources, by searching for appraised evidence, which had been GRADEd, and used the results of high-quality systematic reviews. These sources were often key to ensuring that the response met the required level of urgency, while maintaining the quality of the review.

The rapid review approach based on Cochrane's evolving methodology and building on global repositories of information, demonstrated that urgent assessments of evidence can be done if work is shared freely within the evidence ecosystem and dedicated teams are available. Engagement and co-operation between reviewers and users of the evidence, in this case the SA GRADE Network and the DoH NEMLC sub-committee, are also key to success. Inputs from the sub-committee were constructive and resulted in reporting results in a way that was more easily understood, however, including reviewers in PICO formulation at the outset may have been helpful to ensure feasibility.

The usefulness of review tools varied considerably: while Covidence was an excellent tool for screening and reaching consensus, some databases, such as www.covid-nma.com, were difficult to navigate to find evidence or export content. RoB 2 took considerable time to apply, partially due to reviewers not yet being familiar with its decision rules. It is possible that the Cochrane 'Risk of bias' tool (RoB 1) may have been an acceptable compromise between rigorous Cochrane methodology and urgent response, given that most trials being conducted during the height of the pandemic have considerable methodological shortcomings.[11]

Core outcome sets for COVID-19 should be considered by guideline developers. [12] Some have recently been published,[13] and it would be useful to include these in PICO development. Further duplication should be avoided or minimized by including evidence in network meta-analyses and contributing to evidence ecosystem repositories. In addition, the South African approach draws from current GRADE approaches for developing recommendations as part of urgent responses, [14] however, they could benefit from further investment into evidence-sharing tools, such as GRADEpro GDT for guidelines and MAGICapp used by the Australian national COVID-19 clinical evidence task force (covid19evidence.net. au/\#living-guidelines) in their living systematic reviews.

The nature of the response required the review team to do this work in addition to their usual job. This proved challenging, both for identifying available review authors and to find the time to do the work. This points to inadequate investment in the technical skills needed to support guideline work that needs to be addressed. It has been our experience that reviews with delineated functions within the team, specifically with a clearly identified team lead, progressed faster and more smoothly. There is also a considerable advantage in ensuring continuity, that is, involving a review author with previous rapid review experience, thereby saving considerable time through guidance and understanding of the process. Finally, communication within the team is critical, and quick, frequent touch-base meetings improve team cohesion, ensure timely troubleshooting and avoidance of misunderstandings, and foster accountability. 
Overall, despite the unfortunate crisis brought about by the COVID-19 pandemic, the challenging situation has created a window of policy change. This has enabled new collaborations and improved the use of GRADE methods for reviews and guidelines informing South Africa national guidelines.

\section{Additional resources}

DoH website repository of rapid reviews: http://health.gov.za/index. php/national-essential-medicine-list-committee-nemlc/category/633covid-19-rapid-reviews

COVID-END: mcmasterforum.org/networks/covid-end

\section{Acknowledgements}

NEMLC COVID-19 sub-committee members for collaboration and support.

The South African (SA) GRADE Network (cebhc.co.za/research-what-wedo/south-africa-grade-network), jointly led and managed by the Centre for Evidence-based Health Care (CEBHC), Stellenbosch University, and Cochrane South Africa (CSA), South African Medical Research Council, was formally launched in March 2019. The Network aims to bring together GRADE contributors within South Africa; find consensus on priorities and strategies for advancing GRADE activities in the country; and build on the extensive research and training experience of members of the Network, to co-ordinate and maximize GRADE training, research and guideline development activities in South Africa.

The National Essential Medicine List Committee (NEMLC), an advisory committee appointed by the Minister of Health, is tasked with developing and managing the national essential medicines list as well as standard treatment guidelines for the South African National Department of Health (NDoH). In March 2020, as part of the NDoH's response to the COVID-19 pandemic, a NEMLC COVID-19 sub-committee was established to review, appraise and synthesize emerging evidence, within very short timelines, to inform national standard treatment guidelines for COVID-19.

\section{Declarations of interest}

The authors declare that they have no competing interests. AH, SD and TK are staff at Cochrane South Africa, South African Medical Research Council. SD and TK are co-directors of Cochrane Africa. SD is co-director of Cochrane Nutrition. AB, MM and TY are staff at CEBHC, Division of Epidemiology and Biostatistics, Stellenbosch University. TK is a member of the NEMLC Committee and the NEMLC COVID-19 sub-committee. All authors are members of the SA GRADE Network.

\section{Funding}

We are supported by the Research, Evidence and Development Initiative (READ-It). READ-It (project number 300342-104) is funded by UK aid from the UK government; however, the views expressed do not necessarily reflect the UK government's official policies.

\section{References}

1. World Health Organization. Pneumonia of unknown cause - China: disease outbreak news. Geneva: World Health Organization. 5 January 2020. who.int/csr/don/05-january-2020-pneumonia-of-unkown-causechina.
2. Thayer KA, Schünemann HJ. Using GRADE to respond to health questions with different levels of urgency. Environment International 2016;92-93:585-9. https://doi.org/10.1016/j.envint.2016.03.027

3. Pan American Health Organization. Understanding the infodemic and misinformation in the fight against COVID-19. Factsheet No.5. Geneva: Pan American Health Organization 2020. Available at iris. paho.org/bitstream/handle/10665.2/52052/Factsheet-infodemic_eng. pdf? sequence $=14$.

4. Garritty C, Gartlehner G, Nussbaumer-Streit B, King VJ, Kamel C, Stevens A, et al. Rapid review methods guidance aids in Cochrane's quick response to the COVID-19 crisis. In: Collaborating in response to COVID-19: editorial and methods initiatives across Cochrane. Cochrane Database of Systematic Reviews 2020;(12 Suppl 1). https://doi. org/10.1002/14651858.CD202002

5. Grimshaw JM, Tovey DI, Lavis JN, on behalf of COVID-END. COVIDEND - an international network to better co-ordinate and maximize the impact of the global evidence synthesis and guidance response to COVID-19. In: Collaborating in response to COVID-19: editorial and methods initiatives across Cochrane. Cochrane Database of Systematic Reviews 2020;(12 Suppl 1). https://doi.org/10.1002/14651858.CD202002

6. Covidence, Veritas Health Innovation, Melbourne, Australia. Available at covidence.org.

7. Sackett DL. Rules of evidence and clinical recommendations on the use of antithrombotic agents. Chest 1989;95:2S-4S.

8. Boutron I, Tovey DI, De Nale L, Chaimani A, Devane D, Meerpohl JJ, et al. COVID-NMA: a collaborative COVID-19 living evidence project. In: Collaborating in response to COVID-19: editorial and methods initiatives across Cochrane. Cochrane Database of Systematic Reviews 2020;(12 Suppl 1). https://doi.org/10.1002/14651858.CD202002

9. Sterne JA, Savović J, Page MJ, Elbers RG, Blencowe NS, Boutron I, et al. RoB 2: a revised tool for assessing risk of bias in randomised trials. BMJ 2019;366:14898.

10. GRADEpro GDT. Hamilton (ON): McMaster University (developed by Evidence Prime). Available at gradepro.org.

11. Tricco AC, Garritty CM, Boulos L, Lockwood C, Wilson M, McGowan $J$, et al. Rapid review methods more challenging during COVID-19: commentary with a focus on 8 knowledge synthesis steps. Journal of Clinical Epidemiology 2020;126:177-83. https://doi.org/10.1016/j. jclinepi.2020.06.029

12. Avery K, Macefield R, Gorst S, Harman N, Dodd S, Clarke M, et al. Developing a core outcome set for COVID-19 prevention interventions: the COS COVID-P study. In: Working together in response to COVID-19: Editorial and methods initiatives across Cochrane. Cochrane Database of Systematic Reviews 2020;(12 Suppl 1): https://doi. org/10.1002/14651858.CD202002

13. Williamson P, Gargon L, Clarke M, Marshall J, Zhang J, Qiu R, et al. Core outcome set developers' response to COVID-19. COMET Initiative. 7 July 2020. comet-initiative.org/Studies/Details/1538

14. Akl EA, Morgan R, Rooney A, Beverly B, Katikireddi SV, Agarwal $A$, et al. Developing trustworthy recommendations as part of an urgent response (1-2 weeks): a GRADE concept paper. Journal of Clinical Epidemiology 2020;September 30. https://doi.org/10.1016/j. jclinepi.2020.09.037 